TRANSACTIONS OF THE

AMERICAN MATHEMATICAL SOCIETY

Volume 356, Number 4, Pages 1315-1383

S 0002-9947(03)03448-2

Article electronically published on October 21, 2003

\title{
THE GEOMETRY OF ANALYTIC VARIETIES SATISFYING THE LOCAL PHRAGMÉN-LINDELÖF CONDITION AND A GEOMETRIC CHARACTERIZATION OF THE PARTIAL DIFFERENTIAL OPERATORS THAT ARE SURJECTIVE ON $\mathcal{A}\left(\mathbb{R}^{4}\right)$
}

\author{
RÜDIGER W. BRAUN, REINHOLD MEISE, AND B. A. TAYLOR
}

\begin{abstract}
The local Phragmén-Lindelöf condition for analytic subvarieties of $\mathbb{C}^{n}$ at real points plays a crucial role in complex analysis and in the theory of constant coefficient partial differential operators, as Hörmander has shown. Here, necessary geometric conditions for this Phragmén-Lindelöf condition are derived. They are shown to be sufficient in the case of curves in arbitrary dimension and of surfaces in $\mathbb{C}^{3}$. The latter result leads to a geometric characterization of those constant coefficient partial differential operators which are surjective on the space of all real analytic functions on $\mathbb{R}^{4}$.
\end{abstract}

\section{INTRODUCTION}

In his basic paper 17, Hörmander proved in 1973 that the surjectivity of a given constant coefficient linear partial differential operator $P(D)$ on the space $\mathcal{A}(\Omega)$ of all real analytic functions on any open convex set $\Omega$ in $\mathbb{R}^{n}$ is characterized by certain estimates of Phragmén-Lindelöf type holding for plurisubharmonic functions on the zero variety $V\left(P_{m}\right)$ of its principal symbol $P_{m}$. When $\Omega=\mathbb{R}^{n}$ he showed that this fact is equivalent to a local Phragmén-Lindelöf estimate, $\mathrm{PL}_{\mathrm{loc}}(\xi)$, holding for all $\xi \in V\left(P_{m}\right) \cap \mathbb{R}^{n},|\xi|=1$. For $n=2$ or $n=3$ the latter condition is equivalent to $V\left(P_{m}\right)$ being locally hyperbolic in the sense of Andersson [1] at these points. Later Zampieri [33] and Braun [5] gave other equivalent geometric conditions for $\mathrm{PL}_{\text {loc }}$ when $n=3$. For $n \geq 4$ local hyperbolicity is sufficient but not necessary and no necessary geometric characterization was known at that time. In the meantime, it was shown by several authors that different variants of Phragmén-Lindelöf estimates for plurisubharmonic functions on algebraic varieties could be used to characterize other properties of constant coefficient partial differential operators (see, e.g., [2], 7], [12], [4, [15, 16, 18, 27, 22], 23, 25], 29], 34]). One of these is also of special interest for complex analysis because it describes the natural extension of the classical Phragmén-Lindelöf Theorem to algebraic varieties. In many of these

Received by the editors July 12, 2002 .

2000 Mathematics Subject Classification. Primary 32C25; Secondary 32U05, 35E10.

The authors gratefully acknowledge support of DAAD and NSF under the program "Projektbezogene Förderung des Wissenschaftleraustausch mit den USA in Zusammenarbeit mit der National Science Foundation" and of the Volkswagen-Stiftung (RiP-program in Oberwolfach). The research of the third-named author was supported in part by the National Science Foundation under grant number DMS 0070725. 
cases, the local Phragmén-Lindelöf condition comes up as a necessary condition to hold at real points. Its precise definition is stated as follows:

An analytic variety $V$ in $\mathbb{C}^{n}$ satisfies $\operatorname{PL}_{\text {loc }}(\xi)$ at $\xi \in V \cap \mathbb{R}^{n}$ if there exist $A>0$ and $r \geq \rho>0$ such that each plurisubharmonic function $u$ on $V \cap B(\xi, r)$ which satisfies

$$
u(z) \leq 1, z \in V \cap B(\xi, r) \text { and } u(z) \leq 0, z \in V \cap \mathbb{R}^{n} \cap B(\xi, r)
$$

necessarily satisfies

$$
u(z) \leq A|\operatorname{Im} z|, \quad z \in V \cap B(\xi, \rho) .
$$

In the present paper we first derive new necessary conditions for pure $k$-dimensional varieties $V$ in $\mathbb{C}^{n}$ to satisfy $\mathrm{PL}_{\text {loc }}(0)$ (see Theorem 3.13 ). Then we show that for analytic curves in $\mathbb{C}^{n}$ and for analytic surfaces in $\mathbb{C}^{3}$, these necessary conditions are sufficient (see Proposition 3.16 and Theorem 5.3). The latter theorem is then applied to derive from Hörmander's result a new characterization of those homogeneous polynomials $P$ in four variables for which $P(D)$ is surjective on $\mathcal{A}\left(\mathbb{R}^{4}\right)$ in terms of geometric conditions on the zero variety $V(P)$ of $P$ (see Theorem 6.9). Moreover, we present algorithms that can be used to decide whether $\mathrm{PL}_{\text {loc }}(0)$ holds for an analytic surface in $\mathbb{C}^{3}$ and whether the zero variety of a homogeneous polynomial in four variables satisfies Hörmander's Phragmén-Lindelöf principle.

To outline the basic ideas of the paper, we first remark that the necessary conditions obtained in Section 3 are consequences of the generally known principle that various Phragmén-Lindelöf conditions for varieties are inherited by appropriately defined limit varieties. However, except for the Phragmén-Lindelöf condition introduced by Hörmander, the converse implication does not hold. The reason for this is that near singular points of the limit variety, the branching behavior of $V$ makes it impossible to pass estimates on plurisubharmonic functions from the limit variety of $V$ to $V$ itself. In this situation, the new idea in our analysis of $\mathrm{PL}_{\text {loc }}$ is to microlocalize the analysis of $V$ in "conoids" (see Definition 2.13) centered around analytic curves in $\mathbb{R}^{n}$ that are close to branch curves of $V$ with respect to most projections. The first step in this method was used in our paper [7] in connection with the concept of quasihomogeneity of a polynomial. In order to iterate this analysis, limit varieties $T_{\gamma, d} V$ of $V$ along certain real analytic curves $\gamma$ (see Definition 2.2 ) have to be considered. These limit varieties, which give approximations to $V$ of order $d \geq 1$ in conoids around $\gamma$, were introduced and investigated in our paper 9]. They are either empty or algebraic varieties of essentially one dimension less than the dimension of $V$. Using results from this note and inheritance properties for extremal functions from Meise, Taylor, and Vogt [26, we show that the limit varieties $T_{\gamma, d} V$ have to satisfy $\mathrm{PL}_{\text {loc }}$ at every real point and that furthermore $V$ has to satisfy a hyperbolicity condition in some conoid around $\gamma$ of opening exponent $d$ (see Definition 2.13). We refer to these properties as hyperbolicity in conoids (see Definition 3.14 ). It is a necessary condition for each pure $k$-dimensional variety in $\mathbb{C}^{n}$ to satisfy $\mathrm{PL}_{\text {loc }}(0)$.

For surfaces $V$ in $\mathbb{C}^{3}$ we prove that hyperbolicity in conoids is sufficient for $\mathrm{PL}_{\text {loc }}(0)$. In fact, only a finite number of limit varieties $T_{\gamma, d} V$ have to be used to prove sufficiency (see Theorem 5.3). To obtain this finiteness result, one has to understand exactly which curves and limit varieties have to be inspected to set up an inductive procedure. To show that it stops after finitely many steps in a situation where all relevant necessary conditions have been found, we prove that 
there is a universal denominator $q \in \mathbb{N}$ such that for each $\xi \in \mathbb{R}^{3},|\xi|=1$, all curves in the branch locus $B_{\xi} \subset V$ for the projection of $V$ along $\xi$ have a Puiseux series expansion of the form $\sum_{j=q}^{\infty} a_{j} t^{j / q}, a_{j} \in \mathbb{C}^{3}$.

Another basic ingredient for proving the sufficiency is an application of our main result from [8] concerning the radial local Phragmén-Lindelöf condition. It shows that each plurisubharmonic function $u$ which satisfies the hypotheses of the condition $\mathrm{PL}_{\mathrm{loc}}(0)$ can already be estimated by

$$
u(z) \leq A|z|, \quad z \in V \cap B\left(0, r_{0}\right),
$$

where $A \geq 1$ and $r_{0}>0$ only depend on $V$ near the origin. To derive from this fact and the hyperbolicity in conoids that $V$ satisfies $\mathrm{PL}_{\text {loc }}(0)$, a lot of detailed analysis in conoids has to be done. The main point in it is to understand how properties of the limit varieties $T_{\gamma, d} V$ can be used to get information about $V$.

It turns out that multiplicities in the tangent variety $T_{0} V$ and in the limit varieties $T_{\gamma, d} V$ are responsible for a lot of technical difficulties. However, if $V$ is an analytic surface in $\mathbb{C}^{3}$ which contains the origin and satisfies

$$
\text { all the limit varieties } T_{\gamma, d} V \text { have multiplicity } 1
$$

(see Definition 2.10), then the characterization of $\mathrm{PL}_{\mathrm{loc}}(0)$ for $V$ is essentially obtained by a reduction in dimension and can be stated as follows (see Theorem $7.3)$ :

Theorem. Let $V$ be an analytic surface in $\mathbb{C}^{3}$ which is the zero set of an analytic function with real Taylor coefficients and assume that $V$ contains the origin and satisfies $(*)$. Then $V$ satisfies $\mathrm{PL}_{\mathrm{loc}}(0)$ if and only if all limit varieties $T_{\gamma, d} V$ satisfy $\mathrm{PL}_{\mathrm{loc}}(\xi)$ at each $\xi \in T_{\gamma, d} V \cap \mathbb{R}^{3}$.

In fact, it is enough to know $(*)$ and the condition in the Theorem only for a finite number of limit varieties.

The proof of the characterization also has the following philosophical aspect: No matter how complicated an analytic surface in $\mathbb{C}^{3}$ is, if the local Phragmén-Lindelöf condition can be shown at all, the proof does not bring any new type of maximum principle into play. In fact it uses only two main ingredients. The first one is the local radial Phragmén-Lindelöf condition from [8] in the spirit of the SibonyWong inequality that was already mentioned three paragraphs earlier. It is used to derive an a priori estimate of radial type. Apart from this, only classical estimates from potential theory in the plane are used, although after many localization and reduction steps. This is the essential role that hyperbolicity plays in the analysis.

To apply the characterization of $\mathrm{PL}_{\mathrm{loc}}(0)$ for surfaces in $\mathbb{C}^{3}$ to Hörmander's characterization for polynomials $P$ in four variables, we first show that the following reduction of dimension can be achieved: If $\xi \in V(P) \cap \mathbb{R}^{4},|\xi|=1$ is given, then $V(P)$ satisfies $\mathrm{PL}_{\mathrm{loc}}(\xi)$ if and only if the two-dimensional variety, obtained by slicing $V(P)$ with a hyperplane through $\xi$ and orthogonal to $\xi$, satisfies $\mathrm{PL}_{\text {loc }}(0)$ (see Lemma 6.1). Using an argument of Braun [5], it can be shown that only a finite number of such sliced varieties have to be inspected in order to decide whether $P(D)$ is surjective on $\mathcal{A}\left(\mathbb{R}^{4}\right)$ (see Theorem 6.9).

The paper is organized as follows. In Section 2, we introduce the definitions and results on limit varieties and other things that are needed later. Necessary conditions for $\mathrm{PL}_{\mathrm{loc}}$ are derived in Section 3 and it is shown that they are sufficient for analytic curves in $\mathbb{C}^{n}$ to satisfy $\mathrm{PL}_{\mathrm{loc}}(0)$. In Section 4 the concepts and results 
are presented which are used in Section 5 to prove the finiteness result and the characterization of the analytic surfaces in $\mathbb{C}^{3}$ which satisfy $\mathrm{PL}_{\text {loc }}(0)$. This result is applied in Section 6 to derive a new characterization for the surjectivity of $P(D)$ on $\mathcal{A}\left(\mathbb{R}^{4}\right)$. In Section 7 we prove the Theorem stated above and we illustrate our results by several examples.

In concluding we mention that the results and methods of this paper are used in 11] to characterize those algebraic surfaces in $\mathbb{C}^{n}$ on which the classical PhragménLindelöf theorem holds.

\section{Preliminaries, Real simple Curves, Limit varieties, Conoids}

In this section we fix the notation for the subsequent ones and we recall the basic facts about limit varieties along analytic curves from our article [9].

Throughout this paper $|\cdot|$ will denote the Euclidean norm on $\mathbb{C}^{n}$. At some places it is more convenient to use other norms, which will be mentioned explicitly. For $a \in \mathbb{C}^{n}$ and $r>0$ let

$$
B^{n}(a, r):=\left\{z \in \mathbb{C}^{n}:|z-a|<r\right\},
$$

where the upper index $n$ is often omitted.

An analytic variety $V$ in $\mathbb{C}^{n}$ is defined to be a closed analytic subset of some open set in $\mathbb{C}^{n}$ (see Chirka 14], 2.1). By $V_{\text {sing }}$ (resp. $V_{\text {reg }}$ ) we denote the set of all singular (resp. regular) points in $V$.

2.1. Tangent cones and localizations. Let $V \subset \mathbb{C}^{n}$ be analytic in a neighborhood of $p \in V$. Then $v \in \mathbb{C}^{n}$ is tangent to $V$ at $p$ if there are a sequence $\left(p_{j}\right)_{j}$ in $V$ and a sequence $\left(a_{j}\right)_{j}$ in $\mathbb{C}$ such that $\lim _{j \rightarrow \infty} p_{j}=p$ and $\lim _{j \rightarrow \infty} a_{j}\left(p_{j}-p\right)=v$. The set of all tangent vectors forms a complex cone. It is called the tangent cone of $V$ in $p$ and is denoted by $T_{p} V$.

If $f$ is a holomorphic function in $n$ variables, its localization in $p \in \mathbb{C}^{n}$ is defined as the lowest order nonvanishing term of the expansion $f(\zeta+p)=\sum_{j=0}^{\infty} \sum_{|\alpha|=j} a_{\alpha} \zeta^{\alpha}$. It is denoted by $f_{p}$.

The relation between tangents and localization is expressed by the following fact, which can be found in Whitney [32], Chapter 7, Theorem 4D:

$T_{p} V=\left\{z \in \mathbb{C}^{n}: f_{p}(z)=0\right.$ for all holomorphic functions $f$ vanishing on $\left.V\right\}$.

2.2. Definition. A simple curve $\gamma$ in $\mathbb{C}^{n}$ is a map $\left.\gamma:\right] 0, \alpha\left[\rightarrow \mathbb{C}^{n}\right.$ which for some $\alpha>0$ and some $q \in \mathbb{N}$ admits a convergent Puiseux series expansion

$$
\gamma(t)=\sum_{j=q}^{\infty} \xi_{j} t^{j / q} \text { with }\left|\xi_{q}\right|=1
$$

Then $\xi_{q}$ is called the tangent vector to $\gamma$ in the origin. The trace of $\gamma$ is defined as $\operatorname{tr}(\gamma):=\gamma(] 0, \alpha[)$. A real simple curve is a simple curve $\gamma$ satisfying $\operatorname{tr}(\gamma) \subset \mathbb{R}^{n}$.

2.3. Remark. (a) If $\gamma:] 0, \alpha\left[\rightarrow \mathbb{C}^{n}\right.$ is a simple curve, then for some $0<\beta \leq \alpha$, the restriction of $\gamma$ to $] 0, \beta$ [ is injective. Hence it is no restriction to assume that simple curves are injective.

(b) Each simple curve $\gamma:] 0, \alpha\left(\rightarrow \mathbb{C}^{n}\right.$ has a natural analytic extension to $B(0, \alpha) \backslash$ ]$-\infty, 0]$ if we define for any real number $d>0$ the power function $z^{d}$ on $\left.\left.\mathbb{C} \backslash\right]-\infty, 0\right]$ by $z^{d}=|z|^{d} \exp (i d \arg (z))$, where $\left.\arg (z) \in\right]-\pi, \pi[$. 
2.4. Definition. A real simple curve $\gamma$ in $\mathbb{R}^{n}$ is said to be in standard parametrization with respect to a basis $\left(\xi_{1}, \ldots, \xi_{n}\right)$ of $\mathbb{R}^{n}$ if for some $q \in \mathbb{N}, \gamma(t)=t \xi_{1}+$ $\sum_{\nu=2}^{n} \gamma_{\nu}(t) \xi_{\nu}$, where $\gamma_{\nu}(t)=\sum_{j=q+1}^{\infty} a_{\nu, j} t^{j / q}$.

2.5. Lemma. (a) Let $V \subset \mathbb{C}^{n}$ be a pure 1-dimensional analytic variety in $\mathbb{C}^{n}$ which contains the origin and let $T$ be the germ of a branch of $V \cap \mathbb{R}^{n} \backslash\{0\}$. Then there exists a basis $\left(\xi_{1}, \ldots, \xi_{n}\right)$ of $\mathbb{R}^{n}, r>0$, and a real simple curve $\gamma$ in standard parametrization such that $T \cap B^{n}(0, r)=\operatorname{tr}(\gamma)$.

(b) Let $\gamma$ be a real simple curve in $\mathbb{R}^{n}$ and let $\left(\xi_{1}, \ldots, \xi_{n}\right)$ be a basis of $\mathbb{R}^{n}$ such that $\xi_{1}$ is tangent to $\gamma$. Then there are $\epsilon>0$ and a real simple curve $\delta$ which is in standard parametrization with respect to $\left(\xi_{1}, \ldots, \xi_{n}\right)$ such that $\operatorname{tr}(\gamma) \cap B(0, \epsilon)=\operatorname{tr}(\delta)$.

Proof. Part (a) follows immediately from part (b) together with the well-known result that each irreducible analytic curve admits a Puiseux series expansion (see, e.g., Chirka [14], 6.1).

For the proof of part (b), let $\gamma=\sum_{j=q}^{\infty} a_{j} t^{j / q}$ be the Puiseux series expansion of $\gamma$ satisfying $\left|a_{q}\right|=1$. We may assume $a_{q}=(1,0, \ldots, 0)$ and that $\left(\xi_{1}, \ldots, \xi_{n}\right)$ is the standard basis of $\mathbb{R}^{n}$. Denote by $a_{j, 1}$ the first coordinate of $a_{j}$. Then $g: s \mapsto$ $\sum_{j=q}^{\infty} a_{j, 1} s^{j}$ is analytic near the origin. Let $\phi$ be defined by $g(\phi(s)) \equiv s^{q}$. Then $\phi$ admits a Puiseux series expansion $([14], 6.1)$ of the form $\phi(s)=s+o(s)$. Define

$$
\delta(t):=\gamma\left(\phi\left(t^{1 / q}\right)^{q}\right) .
$$

Then $\operatorname{tr}(\gamma)$ and $\operatorname{tr}(\delta)$ coincide in a neighborhood of the origin. Denote by $\gamma_{1}$ and $\delta_{1}$ the respective first components. Then

$$
\delta_{1}(t)=\gamma_{1}\left(\phi\left(t^{1 / q}\right)^{q}\right)=g\left(\phi\left(t^{1 / q}\right)\right)=t .
$$

Hence $\delta$ is in standard parametrization with respect to the standard basis of $\mathbb{R}^{n}$.

2.6. Definition. Let $V \subset \mathbb{C}^{n}$ be an analytic variety of pure dimension $k \geq 1$ which contains the origin, let $\gamma:] 0, \alpha\left[\rightarrow \mathbb{C}^{n}\right.$ be a simple curve, and let $d \geq 1$. Then for $t \in] 0, \alpha[$ we define

$$
V_{\gamma, t, d}:=\left\{w \in \mathbb{C}^{n}: \gamma(t)+w t^{d} \in V\right\}=\frac{1}{t^{d}}(V-\gamma(t))
$$

and we define the limit variety $T_{\gamma, d} V$ of $V$ of order $d$ along $\gamma$ as the set

$$
\begin{array}{r}
T_{\gamma, d} V:=\left\{\zeta \in \mathbb{C}^{n}: \zeta=\lim _{j \rightarrow \infty} z_{j}, \text { where } z_{j} \in V_{\gamma, t_{j}, d} \text { for } j \in \mathbb{N} \text { and }\left(t_{j}\right)_{j \in \mathbb{N}}\right. \text { is a } \\
\text { null-sequence in }] 0, \alpha[\} .
\end{array}
$$

If it is clear from the context we will sometimes write $V_{t, d}$ or just $V_{t}$ instead of $V_{\gamma, t, d}$.

From Theorem 3.2 and Proposition 4.1 of [9], we recall the following results.

2.7. Theorem. Let $V$ be an algebraic variety of pure dimension $k \geq 1$ which contains the origin, let $\gamma:] 0, \alpha\left[\rightarrow \mathbb{C}^{n}\right.$ be a simple curve in $\mathbb{C}^{n}$ with tangent vector $\xi$ at the origin, and let $d \geq 1$ be given. Then the following assertions hold:

(a) $T_{\gamma, d} V$ is either empty or an algebraic variety of pure dimension $k$.

(b) $T_{\gamma, 1} V=T_{0} V-\xi$.

(c) If $d>1$, then $w \in T_{\gamma, d} V$ if and only if $w+\lambda \xi \in T_{\gamma, d} V$ for each $\lambda \in \mathbb{C}$. 
(d) For each $R>0$ there exists $0<\delta \leq \alpha$ such that $V_{\gamma, t, d}$ is a closed analytic set of $B(0, R)$ for $0<t<\delta$, and for each null-sequence $\left(t_{j}\right)_{j \in \mathbb{N}}$ in $] 0, \delta[$ the varieties $\left(V_{\gamma, t_{j}, d} \cap B(0, R)\right)_{j \in \mathbb{N}}$ converge to $T_{\gamma, d} V \cap B(0, R)$ in the sense of Meise, Taylor, and Vogt 24], 4.3.

For later applications of part (d) of Theorem 2.7 we need the following lemma.

2.8. Lemma. Let $D$ be a domain in $\mathbb{C}^{n}$, let $V_{0}$ and $\left(V_{t}\right)_{t \in] 0, \alpha[}$ be analytic subvarieties of $D$ of pure dimension $k \geq 1$. Assume that for each null-sequence $\left(t_{j}\right)_{j \in \mathbb{N}}$ in ] $0, \alpha\left[\right.$ the sequence $\left(V_{t_{j}}\right)_{j \in \mathbb{N}}$ converges to $V_{0}$ in the sense of Meise, Taylor, and Vogt 24], Definition 4.3. Then for each compact set $K$ in $D$ and each open set $G$ in $D$ with $G \supset V_{0} \cap K$ there exists $0<\tau<\alpha$ such that $V_{t} \cap K \subset G$ for all $\left.t \in\right] 0, \tau[$.

Proof. If the lemma does not hold, then there exist a compact set $K$ in $D$ and an open set $G$ with $K \cap V_{0} \subset G \subset D$ such that for each $j \in \mathbb{N}$ there exists $\left.t_{j} \in\right] 0, \frac{1}{j}$ [ satisfying $K \cap V_{t_{j}} \not \subset G$. Consequently we can choose $z_{j} \in K \cap V_{t_{j}} \backslash G, j \in \mathbb{N}$. Passing to a subsequence, we may assume that $\left(z_{j}\right)_{j \in \mathbb{N}}$ converges to some $z_{0} \in K$. Then the hypothesis implies $z_{0} \in K \cap V_{0} \subset G$. Hence there exists $j_{0} \in \mathbb{N}$ such that $z_{j} \in G$ for all $j \geq j_{0}$. Since this contradicts the choice of the sequence $\left(z_{j}\right)_{j \in \mathbb{N}}$, the proof is complete.

Later we will also need information about equations defining limit varieties $T_{\gamma, d} V$ and about how $T_{\gamma, d} V$ depends on $d \in[1, \infty$ [ for a given real simple curve $\gamma$. To provide it we recall the following definition from [9], 3.15.

2.9. Definition. For $d \in\left[1, \infty\left[\right.\right.$ and $q \in \mathbb{N}$, a polynomial $P$ in $t^{1 / q}$ and $z_{1}, \ldots, z_{n}$,

$$
P(t, z)=\sum_{j \in N, \beta \in M} a_{j, \beta} t^{j / q} z^{\beta}
$$

is called $d$-quasihomogeneous of $d$-degree $\omega$ if $N \subset \mathbb{N}_{0}$ and $M \subset \mathbb{N}_{0}^{n}$ are not empty and if $a_{j, \beta} \neq 0$ and $\frac{j}{q}+d|\beta|=\omega$ for each $j \in N$ and $\beta \in M$.

Remark. If $P$ as in Definition 2.9 is $d$-quasihomogeneous of $d$-degree $\omega$, then $P\left(\lambda t, \lambda^{d} z\right)=\lambda^{\omega} P(t, z)$ for all $\lambda \in \mathbb{C}$ and $\left.(t, z) \in\right] 0, \infty\left[\times \mathbb{C}^{n}\right.$.

2.10. Definition. For $r>0$ let $f: B^{n}(0, r) \rightarrow \mathbb{C}$ be a holomorphic function which vanishes at the origin. For $q \in \mathbb{N}$ let $\gamma(t)=\sum_{j=q}^{\infty} a_{j} t^{j / q}$ be a simple curve. Then we have the expansion

$$
f(\gamma(t)+z)=\sum_{j, \alpha} a_{j, \alpha} z^{\alpha} t^{j / q}
$$

For $d \geq 1$ let

$$
\omega_{0}:=\omega_{0}(d):=\min \left\{\frac{j}{q}+d|\alpha|: a_{j, \alpha} \neq 0\right\}
$$

and regroup the expansion (2.1) as

$$
f(\gamma(t)+z)=F_{\omega_{0}}(t, z)+\sum_{\omega>\omega_{0}} F_{\omega}(t, z)
$$

where $F_{\omega}$ is a $d$-quasihomogeneous polynomial in $t^{1 / q}$ and $z$ of $d$-degree $\omega$. By [9], Corollary 3.17, we have for $V=V(f)$ :

$$
T_{\gamma, d} V=V\left(F_{\omega_{0}}(1, \cdot)\right) .
$$

We will say that $T_{\gamma, d} V$ has multiplicity 1 if $F_{\omega_{0}}(1, \cdot)$ is square-free. 
Remark. In [9] the limit varieties $T_{\gamma, d} V$ are in fact defined as the supports of limit currents $T_{\gamma, d}[V]$. From [9], Corollary 3.16 and 3.17 it follows that $T_{\gamma, d} V$ has multiplicity 1 in the sense of Definition 2.10 if and only if the current $T_{\gamma, d}[V]$ coincides with the current of integration over $T_{\gamma, d} V$ with weight $\equiv 1$.

Note that by Theorem 2.7(b) we have $T_{0} V=T_{\gamma, 1} V+\xi$ for each simple curve $\gamma$ with tangent vector $\xi$ at the origin. Therefore we also have defined when $T_{0} V$ has multiplicity 1 .

2.11. Remark. Let $r>0$ and let $f: B^{n}(0, r) \rightarrow \mathbb{C}$ be a holomorphic function which vanishes of order $m>0$ at the origin. Let $\gamma(t)=\sum_{j=q}^{\infty} a_{j} t^{j / q}$ be a simple curve. It was shown in [9], Proposition 4.3, that there are $p \in \mathbb{N}, 1 \leq p \leq m+1$, and rational numbers $1=d_{1}<\cdots<d_{p}$, such that for $V:=V(f)$

$$
T_{\gamma, d} V=T_{\gamma, \delta} V \text { whenever } d_{j}<d \leq \delta<d_{j+1}, 1 \leq j<p \text { or } d_{p}<d \leq \delta,
$$

and where the set $\left\{d_{1}, \ldots, d_{p}\right\}$ is minimal with respect to this property. It was also shown that the numbers $d_{j}$ can be computed from the expansion (2.1) by considering the Newton diagram $N$ of its support set $M$ which is defined as

$$
M:=\left\{\left(\frac{j}{q}, l\right): j, l \in \mathbb{N}_{0} \text { and } a_{j, \alpha} \neq 0 \text { for some } \alpha \in \mathbb{N}_{0}^{2},|\alpha|=l\right\} .
$$

2.12. Proposition. Under the hypotheses of Remark 2.11 and using the notation introduced there, the following holds:

(a) If $d_{\nu}<d<d_{\nu+1}$ for some $\nu$ satisfying $1 \leq \nu<p$, then $F_{\omega_{0}(d)}(1, \cdot)=$ $\left(F_{\omega_{0}\left(d_{\nu}\right)}(1, \cdot)\right)_{0}$ and hence

$$
T_{\gamma, d} V=V\left(\left(F_{\omega_{0}\left(d_{\nu}\right)}(1, \cdot)\right)_{0}\right) .
$$

(b) If $d>d_{p}$, then $F_{\omega_{0}(d)}(1, \cdot)=\left(F_{\omega_{0}\left(d_{p}\right)}(1, \cdot)\right)_{0}$ and hence

$$
T_{\gamma, d} V=V\left(\left(F_{\omega_{0}\left(d_{p}\right)}(1, \cdot)\right)_{0}\right) \text {. }
$$

(c) $\operatorname{deg} F_{\omega_{0}\left(d_{\nu+1}\right)}(1, \cdot)<\operatorname{deg} F_{\omega_{0}\left(d_{\nu}\right)}(1, \cdot)$ for $1<\nu<p$.

Proof. To prove (a) and (b) we consider the following cases:

Case 1: $\nu=1$. Expand $f=\sum_{k=m}^{\infty} Q_{m}$, where $Q_{k}$ is either homogeneous of degree $k$ or identically zero. If $Q_{k} \not \equiv 0$ consider the Taylor series expansion of $Q_{k}$ at $a_{q}$ :

$$
Q_{k}\left(a_{q}+w\right)=\sum_{\nu=\nu_{k}}^{k} P_{k, \nu}(w)
$$

where $P_{k, \nu}$ is homogeneous of degree $\nu$ or identically zero and where $P_{k, \nu_{k}}=\left(Q_{k}\right)_{a_{q}}$ is the localization of $Q_{k}$ at $a_{q}$. For $\tilde{\gamma}(t):=\gamma(t)-a_{q} t$ this gives

$$
Q_{k}(\gamma(t)+z)=t^{k} Q_{k}\left(a_{q}+\frac{\tilde{\gamma}(t)+z}{t}\right)=\sum_{\nu=\nu_{k}}^{k} t^{k-\nu} P_{k, \nu}(\tilde{\gamma}(t)+z) .
$$

Since $\tilde{\gamma}(t)=\sum_{j=1}^{\infty} a_{q+j} t^{j / q}$, the expansion of $P_{k, \nu}(\tilde{\gamma}(t)+z)$ shows that the values

$$
k-\nu+\frac{q+1}{q} \cdot j+d(\nu-j)=k+(d-1) \nu+j\left(\frac{q+1}{1}-d\right), 0 \leq j \leq \nu,
$$

are candidates for the smallest $d$-degree in the expansion of $Q_{k}(\gamma(t)+z), k \geq m$. From this it follows that

$$
\omega_{0}(1)=m \text { and } F_{\omega_{0}(1)}(1, \cdot)=P_{m, \nu_{m}}=\left(Q_{m}\right)_{a_{q}} .
$$


Moreover, it follows that for some $\varepsilon>0$ and $1<d<1+\varepsilon$ we have

$$
\omega_{0}(d)=m \text { and } F_{\omega_{0}(d)}(1, \cdot)=P_{m, \nu_{m}}=\left(Q_{m}\right)_{a_{q}} .
$$

By 9], Proposition 4.3, (2.3), and the choice of $d_{2}$, this proves (a) in this case.

Case 2: $1<\nu<p$. By [9], 4.3, there is a segment with slope $-\frac{1}{d_{\nu}}$ in $N$. By the definition of the Newton polygon there are $n \geq 2$ points $\left(\frac{j_{k}}{q}, l_{k}\right) \in M, 1 \leq k \leq n$, lying on this segment, i.e., they satisfy

$$
d_{\nu} l_{k}+\frac{j_{k}}{q}=\omega_{0}\left(d_{\nu}\right), \quad 1 \leq k \leq n
$$

while

$$
d_{\nu} l+\frac{j}{q}>\omega_{0}\left(d_{\nu}\right) \text { for all }\left(\frac{j}{q}, l\right) \in M,\left(\frac{j}{q}, l\right) \neq\left(\frac{j_{k}}{q}, l_{k}\right), 1 \leq k \leq n .
$$

Moreover,

$$
F_{\omega_{0}\left(d_{\nu}\right)}(t, z)=\sum_{k=1}^{n} S_{k}(t, z), \text { where } S_{k}(t, z)=\sum_{d_{\nu}|\alpha|+j_{k} / q=\omega_{0}\left(d_{\nu}\right)} a_{j_{k}, \alpha} z^{\alpha} t^{j_{k} / q} .
$$

In particular, $S_{k}(1, \cdot)$ is homogeneous of degree $l_{k}$ for $1 \leq k \leq n$. Since we may assume $j_{1}<j_{2}<\cdots<j_{n}$, we have $l_{1}>l_{2}>\cdots>l_{n}$. Consequently, the localization of $F_{\omega_{0}\left(d_{\nu}\right)}(1, \cdot)$ at the origin is $S_{n}(1, \cdot)$. To compute $F_{\omega_{0}(d)}$ for $d>d_{\nu}$, note first that the $d$-degree of $S_{k}$ is $d l_{k}+j_{k} / q$. Since $l_{k}=\frac{1}{d_{\nu}}\left(\omega_{0}\left(d_{\nu}\right)-j_{k} / q\right)$, we get for $k<l$

$$
\begin{aligned}
& d l_{k}+\frac{j_{k}}{q}=\frac{d}{d_{\nu}}\left(\omega_{0}\left(d_{\nu}\right)-\frac{j_{k}}{q}\right)+\frac{j_{k}}{q}=\frac{d \omega_{0}\left(d_{\nu}\right)}{d_{\nu}}-\frac{j_{k}}{q}\left(\frac{d}{d_{\nu}}-1\right) \\
&>\frac{d \omega_{0}\left(d_{\nu}\right)}{d_{\nu}}-\frac{j_{l}}{q}\left(\frac{d}{d_{\nu}}-1\right)=d l_{l}+\frac{j_{l}}{q} .
\end{aligned}
$$

Hence, among $S_{1}, \ldots, S_{n}$, the term $S_{n}$ has minimal $d$-degree $d l_{n}+j_{n} / q$. To check the $d$-degree of the remaining points in $M$, note that the set of $\left(\frac{j}{q}, l\right) \in M$ described in (2.4) is either empty or the minimal $d_{\nu}$-degree in this set is attained at some point $\left(\frac{j_{0}}{q}, l_{0}\right)$. Then $(2.4)$ implies

$$
d l_{0}+\frac{j_{0}}{q}>\omega_{0}\left(d_{\nu}\right) \geq d l_{n}+\frac{j_{n}}{q}
$$

for $d=d_{\nu}$. Hence there exists $\varepsilon>0$ such that (2.5) also holds for $d_{\nu}<d<d_{\nu}+\varepsilon$ and consequently

$$
\omega_{0}(d)=d l_{n}+\frac{j_{n}}{q} \text { and } F_{\omega_{0}(d)}=S_{n} \text { for } d_{\nu}<d<d_{\nu}+\varepsilon .
$$

By 9], Proposition 4.3, (2.3), and the above, this implies

$$
T_{\gamma, d} V=V\left(F_{\omega_{0}(d)}(1, \cdot)\right)=V\left(S_{n}(1, \cdot)\right)=V\left(\left(F_{\omega_{0}\left(d_{\nu}\right)}(1, \cdot)\right)_{0}\right) .
$$

Since $T_{\gamma, d} V=T_{\gamma, \delta} V$ for $d_{\nu}<d, \delta<d_{\nu+1}$, we proved (a) in this case.

Case 3: $\nu=p$ and $p>1$. In this case we can argue as in the previous one to get $n \geq 2$ and points $\left(\frac{j_{k}}{q}, l_{k}\right) \in M, 1 \leq k \leq n$, lying on the segment in $N$ with slope $-\frac{1}{d_{p}}$. Assuming $j_{1}<j_{2}<\cdots<j_{n}$ as before, we have

$$
F_{\omega_{0}\left(d_{p}\right)}(t, z)=\sum_{k=1}^{n} S_{k}(t, z), \text { where } S_{k}(t, z)=\sum_{d_{p}|\alpha|+j_{k} / q=\omega_{0}\left(d_{p}\right)} a_{j, \alpha} z^{\alpha} t^{j_{k} / q},
$$


using the same notation as above. The definition of $p$ and of the Newton polygon implies that either there are no points $\left(\frac{j}{q}, l\right)$ in $M$ satisfying $j>j_{n}$ or $l \geq l_{n}$ if $\left(\frac{j}{q}, l\right) \in M$ with $j>j_{n}$. Hence we have

$$
\frac{j}{q}+d l>\frac{j_{n}}{q}+d l_{n} \text { for each such point and } d>d_{p} .
$$

From this we get as above that $\omega_{0}(d)=\frac{j_{n}}{q}+d l_{n}$ for $d>d_{p}$ and $F_{\omega_{0}(d)}=S_{n}$. As before, this implies (b).

(c) The considerations in case 2 above imply

$$
\operatorname{deg} F_{\omega_{0}\left(d_{\nu}\right)}(1, \cdot)=l_{1}>l_{n}=\operatorname{deg} S_{n}(1, \cdot) .
$$

The definition of the Newton polygon gives $F_{\omega_{0}\left(d_{\nu+1}\right)}=S_{n}+R$, where the degree of $R(1, \cdot)$ is smaller than $l_{n}$. Hence (c) holds.

2.13. Definition. Let $\gamma:] 0, \alpha\left[\rightarrow \mathbb{R}^{n}\right.$ be a real simple curve, let $d \geq 1$, a subset $U$ of $\mathbb{C}^{n}$, and $0<R \leq \alpha$ be given. We call

$$
\Gamma(\gamma, d, U, R):=\bigcup_{0<t<R}\left(\gamma(t)+t^{d} U\right)
$$

the conoid with core $\gamma$, opening exponent $d$, and profile $U$, truncated at $R$, provided that the origin does not belong to this set.

2.14. Definition. Two simple curves $\gamma$ and $\sigma$ in $\mathbb{C}^{n}$ are called equivalent modulo $d \geq 1$ if for each zero-neighborhood $U$ in $\mathbb{C}^{n}$ and each $R>0$ for which $\Gamma(\gamma, d, U, R)$ and $\Gamma(\sigma, d, U, R)$ are conoids, we have

$$
\Gamma(\gamma, d, U, R) \cap \Gamma(\sigma, d, U, R) \neq \emptyset .
$$

If $T$ is the germ of a branch of $V \cap \mathbb{R}^{n} \backslash\{0\}$ as in Lemma 2.5 then $T$ is said to be equivalent to $\gamma$ modulo $d$ if there exists a real simple curve $\sigma$ which is equivalent to $\gamma$ modulo $d$ and satisfies $\operatorname{tr}(\sigma)=T$.

2.15. Lemma. Let $\gamma$ and $\beta$ be two real simple curves in $\mathbb{R}^{n}$ defined on $] 0, \alpha[$ for some $\alpha>0$. Then the following assertions hold:

(a) If $\gamma$ and $\beta$ are equivalent modulo $d$ for some $d \geq 1$, then their tangent vectors in the origin coincide.

(b) If $\gamma(t)=\sum_{j=q}^{\infty} a_{j} t^{j / q}$ and $\beta(t)=\sum_{j=l}^{\infty} b_{j} t^{j / l}$ are standard parametrization of $\gamma$ and $\beta$ with respect to the same real basis $\mathfrak{X}$ in the $\mathfrak{X}$-coordinates, then $\gamma$ and $\beta$ are equivalent modulo $d>1$ if and only if

$$
\left.\sum_{j=q}^{d q} a_{j} t^{j / q}=\sum_{j=l}^{d l} b_{j} t^{j / l}, \quad t \in\right] 0, \alpha[.
$$

(c) The relation "equivalence modulo d" defined in Definition 2.14 is an equivalence relation for real simple curves.

Proof. Let $\xi$ denote the tangent vector of $\gamma$ in the origin. It is easy to check that for each sequence $\left(p_{k}\right)_{k \in \mathbb{N}}$ which satisfies $p_{k} \in \Gamma\left(\gamma, d, B\left(0, \frac{1}{k}\right), \frac{1}{k}\right)$ for $k \in \mathbb{N}$, we have $\lim _{k \rightarrow \infty} p_{k} /\left|p_{k}\right|=\xi$. Obviously, this implies (a). 
To prove (b), assume first that (2.7) holds. Then for each $0<\varepsilon<1$ and all small $R>0$ we have

$$
\sum_{j=q}^{d q} a_{j} t^{j / q} \in \Gamma(\gamma, d, B(0, \varepsilon), R) \cap \Gamma(\beta, d, B(0, \varepsilon), R)
$$

provided that $t>0$ is sufficiently small. Hence $\gamma$ and $\beta$ are equivalent modulo $d$.

For the proof of the converse implication, replace $q$ and $l$ by a common multiple if necessary, so that $q=l$. To prove inductively that $a_{j}=b_{j}$ for all $j$ satisfying $q \leq j \leq d q$, note that by part (a) this holds for $j=q$. Assume now that for some $J$ with $q<J \leq d q$ we already know $a_{j}=b_{j}$ for all $q \leq j<J$. Since $\gamma$ and $\beta$ are equivalent modulo $d$, there exists a sequence $\left(p_{k}\right)_{k \in \mathbb{N}}$ satisfying $p_{k} \in$ $\Gamma(\gamma, d, B(0,1 / k), 1 / k) \cap \Gamma\left(\beta, d, B(0,1 / k), \frac{1}{k}\right), k \in \mathbb{N}$. Since $\gamma$ and $\beta$ are in standard parametrization with respect to the same basis, there are null-sequences $\left(t_{k}\right)_{k \in \mathbb{N}}$ and $\left(\tau_{k}\right)_{k \in \mathbb{N}}$ in $] 0, \infty\left[\right.$ and $\left(g_{k}\right)_{k \in \mathbb{N}}$ and $\left(h_{k}\right)_{k \in \mathbb{N}}$ in $\mathbb{C}^{n}$ such that

$$
\gamma\left(t_{k}\right)+g_{k}=p_{k}=\beta\left(\tau_{k}\right)+h_{k},\left|g_{k}\right|<\frac{1}{k} t_{k}^{d},\left|h_{k}\right|<\frac{1}{k} \tau_{k}^{d},
$$

and hence

$$
\left|t_{k}-\tau_{k}\right| \leq\left|g_{k}-h_{k}\right| \leq \frac{1}{k}\left(t_{k}^{d}+\tau_{k}^{d}\right) .
$$

Since $\lim _{k \rightarrow \infty}\left|p_{k}\right| / t_{k}=|\xi|=\lim _{k \rightarrow \infty}\left|p_{k}\right| / \tau_{k}$ and $|\xi|=1$, this implies $\left|t_{k}-\tau_{k}\right| \leq$ $\frac{3}{k}\left|p_{k}\right|^{d}$ for all sufficiently large $k$. Moreover, $J \leq q d$ and $0<t_{k}, \tau_{k}<1$ imply

$$
0 \leq \lim _{k \rightarrow \infty} \frac{\left|g_{k}\right|}{t_{k}^{J / q}} \leq \lim _{k \rightarrow \infty} \frac{\left|g_{k}\right|}{t_{k}^{d}}=0 \quad \text { and } \quad 0 \leq \lim _{k \rightarrow \infty} \frac{\left|h_{k}\right|}{\tau_{k}^{J / q}} \leq \lim _{k \rightarrow \infty} \frac{\left|h_{k}\right|}{\tau_{k}^{d}}=0 .
$$

From this it follows that

$$
\lim _{k \rightarrow \infty} \frac{1}{\left|p_{k}\right|^{J / q}}\left(p_{k}-\sum_{j=q}^{J-1} a_{j} t_{k}^{j / q}\right)=a_{J}, \lim _{k \rightarrow \infty} \frac{1}{\left|p_{k}\right|^{J / q}}\left(p_{k}-\sum_{j=q}^{J-1} b_{j} \tau_{k}^{j / q}\right)=b_{J} .
$$

Since for $q \leq j \leq J-1$ we have

$$
\frac{1}{\left|p_{k}\right|^{J / q}}\left|t_{k}^{j / q}-\tau_{k}^{j / q}\right| \leq \frac{1}{\left|p_{k}\right|^{J / q}} \frac{j}{q} \sup _{s \in[0,1]}\left|t_{k}+s\left(\tau_{k}-t_{k}\right)\right|^{j / q-1} \frac{3\left|p_{k}\right|^{d}}{k},
$$

the induction hypothesis $a_{j}=b_{j}$ for $q \leq j \leq J-1$ implies $a_{J}=b_{J}$, which completes the proof of part (b).

Part (c) is an obvious consequence of (a) and (b).

Later we shall also need the following definition.

2.16. Definition. Let $V$ be an analytic variety in $\mathbb{C}^{n}$ and let $\Omega$ be an open subset of $V$. A function $u: \Omega \rightarrow[-\infty, \infty$ [ is called plurisubharmonic if it is locally bounded above, plurisubharmonic in the usual sense on $\Omega_{\text {reg }}$, the set of all regular points of $V$ in $\Omega$, and satisfies

$$
u(z)=\limsup _{\zeta \in \Omega_{\mathrm{reg}}, \zeta \rightarrow z} u(\zeta)
$$

at the singular points of $V$ in $\Omega$. By $\operatorname{PSH}(\Omega)$ we denote the set of all plurisubharmonic functions on $\Omega$. 


\section{NeCessary COnditions and $(\gamma, d)$-Hyperbolicity}

In this section we will derive necessary conditions for an analytic variety $V$ of pure dimension $k$ in $\mathbb{C}^{n}$ to satisfy $\mathrm{PL}_{\text {loc }}$ at one of its real points. We also show that for $k=1$, this necessary condition is sufficient. The next two sections are then needed to prove the sufficiency of this condition for two-dimensional analytic varieties in $\mathbb{C}^{3}$. First we recall the definition of the local Phragmén-Lindelöf condition $\mathrm{PL}_{\text {loc }}$.

3.1. Definition. For $\xi \in \mathbb{R}^{n}$ and $r_{0}>0$ let $V$ be an analytic variety in $B\left(\xi, r_{0}\right)$ which contains $\xi$. We say that $V$ satisfies the condition $\mathrm{PL}_{\text {loc }}(\xi)$ if there exist positive numbers $A$ and $r_{0} \geq r_{1} \geq r_{2}$ such that each $u \in \operatorname{PSH}\left(V \cap B\left(\xi, r_{1}\right)\right)$ satisfying

( $\alpha) u(z) \leq 1, z \in V \cap B\left(\xi, r_{1}\right)$ and

( $\beta) u(z) \leq 0, z \in V \cap \mathbb{R}^{n} \cap B\left(\xi, r_{1}\right)$

also satisfies

$(\gamma) u(z) \leq A|\operatorname{Im} z|, z \in V \cap B\left(\xi, r_{2}\right)$.

To derive several equivalent formulations for $\mathrm{PL}_{\mathrm{loc}}$, we recall the following lemma from Meise, Taylor, and Vogt [24, 2.9:

3.2. Lemma. The function $H: \mathbb{C}^{n} \rightarrow \mathbb{R}, H(z):=\frac{1}{2}\left(|\operatorname{Im} z|^{2}-|\operatorname{Re} z|^{2}\right)$ is plurisubharmonic and has the following properties:
(a) $H(z) \leq|\operatorname{Im} z|,|z| \leq 1$,
(c) $H(x) \leq 0, x \in \mathbb{R}^{n}$,
(b) $H(z) \leq|\operatorname{Im} z|-\frac{1}{2},|z|=1$,
(d) $H(i y) \geq 0, y \in \mathbb{R}^{n}$.

3.3. Lemma. For $\xi \in \mathbb{R}^{n}$ let $V$ be an analytic variety in $B\left(\xi, r_{0}\right), r_{0}>0$, which contains $\xi$. For positive numbers $A, r_{3}<r_{2} \leq r_{1} \leq r_{0}$, and functions $u$ defined on $V \cap B\left(\xi, r_{1}\right)$, consider the estimates

( $\alpha) u(z) \leq 1, \quad z \in V \cap B\left(\xi, r_{1}\right)$.

( $\beta) u(z) \leq 0, \quad z \in V \cap \mathbb{R}^{n} \cap B\left(\xi, r_{2}\right)$.

$(\gamma) u(z) \leq A|\operatorname{Im} z|, \quad z \in V \cap B\left(\xi, r_{3}\right)$.

Then the following conditions are equivalent:

(a) For each choice of $r_{1}$ and $r_{2}$ there exist $r_{3}$ and $A$ such that for each $u \in$ $\operatorname{PSH}\left(V \cap B\left(\xi, r_{1}\right)\right)$ the estimates $(\alpha)$ and $(\beta)$ imply the estimate $(\gamma)$.

(b) There exist $r_{1}, r_{2}, r_{3}$ and $A$ such that for each $u \in \operatorname{PSH}\left(V \cap B\left(\xi, r_{1}\right)\right)$ the estimates $(\alpha)$ and $(\beta)$ imply the estimate $(\gamma)$.

(c) $V$ satisfies $\mathrm{PL}_{\mathrm{loc}}(\xi)$.

In particular, the set

$$
\left\{\xi \in V \cap \mathbb{R}^{n}: V \text { satisfies } \mathrm{PL}_{\mathrm{loc}}(\xi)\right\}
$$

is open in $V \cap \mathbb{R}^{n}$.

Proof. Obviously, (a) implies (c) and (c) implies (b). Hence it suffices to show that (b) implies (a). To do so, assume that (b) holds with the parameters $0<s_{3}<s_{2} \leq$ $s_{1} \leq r_{0}$ and $A_{0}>0$. Then let $0<r_{2} \leq r_{1} \leq r_{0}$ be given, let

$$
r_{3}:=\min \left(s_{3}, r_{2} / 2\right), B:=\frac{5}{r_{2}}, \text { and } A:=\left(1+\frac{5 s_{1}}{r_{2}}\right) A_{0},
$$


and fix $u \in \operatorname{PSH}\left(V \cap B\left(\xi, r_{1}\right)\right)$ satisfying $(\alpha)$ and $(\beta)$. To show that $u$ also satisfies $(\gamma)$, fix $z_{0} \in V \cap B\left(\xi, r_{3}\right)$ and define $w: V \rightarrow[-\infty, \infty[$ by

$$
w(z):= \begin{cases}\max \left\{u(z)+B \frac{r_{2}}{2} H\left(\frac{2\left(z-\operatorname{Re} z_{0}\right)}{r_{2}}\right), B|\operatorname{Im} z|\right\}, & \left|z-\operatorname{Re} z_{0}\right|<\frac{r_{2}}{2}, \\ B|\operatorname{Im} z|, & \text { otherwise. }\end{cases}
$$

To see that $w$ is plurisubharmonic on $V$, note that by 3.2(b) we have for $z \in V$ satisfying $\left|z-\operatorname{Re} z_{0}\right|=\frac{r_{2}}{2}$ :

$$
\begin{aligned}
u(z)+B \frac{r_{2}}{2} H\left(\frac{2\left(z-\operatorname{Re} z_{0}\right)}{r_{2}}\right) & \leq 1+B \frac{r_{2}}{2}\left(\left|\operatorname{Im} \frac{2 z}{r_{2}}\right|-\frac{1}{2}\right) \\
& =B|\operatorname{Im} z|+1-B \frac{r_{2}}{4}<B|\operatorname{Im} z| .
\end{aligned}
$$

It is easy to check that from $(\alpha)$ for $u, 3.2(\mathrm{a})$, and the definition of $w$ we get the estimate

$$
w(z) \leq 1+B s_{1}, \quad z \in V \cap B\left(\xi, s_{1}\right) .
$$

Since $u$ satisfies $(\beta)$ and since $z \in V$ and $\left|z-\operatorname{Re} z_{0}\right| \leq \frac{r_{2}}{2}$ imply $z \in V \cap B\left(\xi, r_{2}\right)$, property $3.2(\mathrm{c})$ implies $w(z) \leq 0$ for $z \in V \cap \mathbb{R}^{n}$. Hence the present hypothesis implies

$$
w(z) \leq A_{0}\left(1+B s_{1}\right)|\operatorname{Im} z|, \quad z \in V \cap B\left(\xi, s_{3}\right) .
$$

By the definition of $r_{3}$, we have $r_{3} \leq s_{3}$ and

$$
\left|z_{0}-\operatorname{Re} z_{0}\right|=\left|\operatorname{Im} z_{0}\right| \leq\left|z_{0}\right|<r_{3} \leq \frac{r_{2}}{2} .
$$

Consequently, the definition of $w$ implies by $3.2(\mathrm{~d})$ :

$$
A_{0}\left(1+B s_{1}\right)\left|\operatorname{Im} z_{0}\right| \geq w\left(z_{0}\right) \geq u\left(z_{0}\right)+B \frac{r_{2}}{2} H\left(\frac{2 i \operatorname{Im} z_{0}}{r_{2}}\right) \geq u\left(z_{0}\right)
$$

and hence

$$
u\left(z_{0}\right) \leq A_{0}\left(1+\frac{5 s_{1}}{r_{2}}\right)\left|\operatorname{Im} z_{0}\right|=A\left|\operatorname{Im} z_{0}\right|, z_{0} \in V \cap B\left(\xi, r_{3}\right) .
$$

To prove the last statement of the lemma, assume that $V$ satisfies $\mathrm{PL}_{\text {loc }}\left(\xi_{0}\right)$ for some $\xi_{0} \in V \cap \mathbb{R}^{n}$ and choose $\rho_{1}>0$ such that $V$ is a closed analytic subset of $B\left(\xi_{0}, 2 \rho_{1}\right)$. Then let $r_{1}:=r_{2}:=\rho_{1} / 2$ and apply (a) to get $0<r_{3}<r_{2}$ and $A>0$ such that the estimates $(\alpha)$ and $(\beta)$ at $\xi_{0}$ imply $(\gamma)$ for $u \in \operatorname{PSH}\left(V \cap B\left(\xi_{0}, r_{1}\right)\right)$. We claim that for each $\xi \in V \cap \mathbb{R}^{n}$ satisfying

$$
\left|\xi-\xi_{0}\right|<\min \left(\frac{\rho_{1}}{2}, \frac{r_{3}}{2}\right)
$$

$V$ satisfies $\mathrm{PL}_{\text {loc }}(\xi)$. To prove this, fix such a point $\xi$ and $\varphi \in \operatorname{PSH}\left(V \cap B\left(\xi, \rho_{1}\right)\right)$ satisfying

$$
\varphi(z) \leq 1, z \in V \cap B\left(\xi, \rho_{1}\right) \text { and } \varphi(z) \leq 0, z \in V \cap \mathbb{R}^{n} \cap B\left(\xi, \rho_{1}\right) .
$$

By our choices we have $B\left(\xi_{0}, r_{1}\right) \subset B\left(\xi, \rho_{1}\right)$. Hence $u:=\left.\varphi\right|_{V \cap B\left(\xi_{0}, r_{1}\right)}$ satisfies $(\alpha)$ and $(\beta)$ for $\xi_{0}$ and consequently

$$
\varphi(z)=u(z) \leq A|\operatorname{Im} z|, \quad z \in V \cap B\left(\xi_{0}, r_{3}\right) .
$$

Of course, this implies

$$
\varphi(z) \leq A|\operatorname{Im} z|, \quad z \in V \cap B\left(\xi, r_{3} / 2\right) .
$$

Hence $V$ satisfies $\mathrm{PL}_{\mathrm{loc}}(\xi)$ because of $(\mathrm{b})$. 
3.4. Lemma. Let $V$ be an analytic variety of pure dimension $k$ in $\mathbb{C}^{n}$ satisfying $0 \in V$ and let $d \geq 1$ and a real simple curve $\gamma:] 0, \alpha\left[\rightarrow \mathbb{R}^{n}\right.$ be given. If $V$ satisfies $\mathrm{PL}_{\mathrm{loc}}(0)$, then the following holds:

For each open set $D$ in $\mathbb{C}^{n}$ and each compact subset $K$ of $D$ there exist $A_{0}$ and $0<t_{0}<\alpha$ such that each family $\left(u_{t}\right)_{t \in] 0, \alpha[}$ of plurisubharmonic functions $u_{t}$ on $V_{t, d} \cap D$ which satisfies

$\left.(\alpha) u_{t}(z) \leq 1, z \in V_{t, d} \cap D, t \in\right] 0, \alpha[$ and

( $\left.\beta) u_{t}(z) \leq 0, z \in V_{t, d} \cap D \cap \mathbb{R}^{n}, t \in\right] 0, \alpha[$

also satisfies

$(\gamma) u_{t}(z) \leq A_{0}|\operatorname{Im} z|, z \in V_{t, d} \cap K, 0<t \leq t_{0}$.

Proof. To prove the lemma fix an open set $D$ in $\mathbb{C}^{n}$ and a compact subset $K$ of $D$. Then choose $\eta$ with $0<\eta<1$ so that $K+B(0,2 \eta) \subset D$. Since $V$ satisfies $\mathrm{PL}_{\mathrm{loc}}(0)$ by hypothesis, there are positive numbers $A, r_{1}$, and $r_{2}$ according to Definition 3.1. Choose $0<t_{0}<\alpha$ so that

$$
\begin{aligned}
& \left\{\gamma(t)+t^{d} z: 0<t<t_{0}, z \in K\right\} \subset B\left(0, r_{2}\right), \\
& \gamma(t) \neq 0 \text { for } 0<t \leq t_{0}, \text { and } \frac{\eta}{2} t_{0}^{d}+r_{1}<2 r_{1},
\end{aligned}
$$

and let $A_{0}:=\max \left(\frac{4 A r_{1}}{\eta}, \frac{1}{\eta}\right)$. Then fix an arbitrary family $\left(u_{t}\right)_{t \in] 0, \alpha[}$ of plurisubharmonic functions which have the properties given in the lemma. To prove $(\gamma)$ fix an arbitrary point $z_{0} \in V_{t, d} \cap K$.

If $\left|\operatorname{Im} z_{0}\right| \geq \eta$, then condition $(\alpha)$ implies

$$
u_{t}\left(z_{0}\right) \leq 1 \leq \frac{1}{\eta}\left|\operatorname{Im} z_{0}\right| \leq A_{0}\left|\operatorname{Im} z_{0}\right| .
$$

Hence $(\gamma)$ holds in this case.

If $\left|\operatorname{Im} z_{0}\right|<\eta$, then we will apply a localization argument as in the proof of Lemma 3.3. To do so note that for each $0<t<\alpha$ the map

$$
F_{t, d}: V_{t, d} \rightarrow V, \quad F_{t, d}(z)=\gamma(t)+t^{d} z,
$$

is a holomorphic bijection by the definition of $V_{t, d}$. Moreover, for $z \in V_{t, d}$ with $\left|z-\operatorname{Re} z_{0}\right| \leq \eta$ the choice of $\eta$ implies

$$
z=z-\operatorname{Re} z_{0}-i \operatorname{Im} z_{0}+z_{0} \in K+B(0,2 \eta) \subset D,
$$

since $\left|\operatorname{Im} z_{0}\right|<\eta$. Hence we can define a map $\varphi_{t}: V \rightarrow \mathbb{R}$ as follows: If $w=$ $\gamma(t)+t^{d} z \in V$ and $\left|z-\operatorname{Re} z_{0}\right|<\eta$, then denote by $H$ the function from Lemma 3.2 and let

$$
\varphi_{t}(w):=t^{d} \max \left\{\frac{\eta}{2}\left(u_{t}(z)+2 H\left(\frac{z-\operatorname{Re} z_{0}}{\eta}\right)\right),|\operatorname{Im} z|\right\} .
$$

Otherwise define

$$
\varphi_{t}(w):=|\operatorname{Im} w| .
$$

It is easy to check that $\varphi_{t}$ is plurisubharmonic on $V$ since for each point $w=$ $\gamma(t)+t^{d} z$ in the boundary with respect to $V$ of the set

$$
\left\{\gamma(t)+t^{d} z: z \in V_{t, d},\left|z-\operatorname{Re} z_{0}\right|<\eta\right\},
$$


condition $(\alpha)$ and the property 3.2(b) of $H$ imply the following estimate:

$$
\begin{aligned}
t^{d} \max & \left\{\frac{\eta}{2}\left(u_{t}(z)+2 H\left(\frac{z-\operatorname{Re} z_{0}}{\eta}\right)\right),|\operatorname{Im} z|\right\} \\
& \leq t^{d} \max \left\{\frac{\eta}{2}\left(1+2\left|\operatorname{Im}\left(\frac{z-\operatorname{Re} z_{0}}{\eta}\right)\right|-1\right),|\operatorname{Im} z|\right\} \\
& =t^{d}|\operatorname{Im} z|=t^{d}\left|\operatorname{Im}\left(\frac{\gamma(t)}{t^{d}}+z\right)\right|=|\operatorname{Im} w| .
\end{aligned}
$$

Next note that for $w \in V \cap B\left(0, r_{1}\right)$ the definition of $\varphi$ and the property 3.2(a) of $H$ imply

$$
\varphi_{t}(w) \leq \max \left(t^{d} \frac{\eta}{2}+r_{1}, r_{1}\right) \leq 2 r_{1},
$$

by our choice of $\eta$ and $t_{0}$. From condition $(\beta)$, the definition of $\varphi_{t}$, and the property $3.2(\mathrm{c})$ of $H$ it follows that

$$
\varphi_{t}(w) \leq 0, w \in V \cap B\left(0, r_{1}\right) \cap \mathbb{R}^{n} .
$$

Since $V$ satisfies $\mathrm{PL}_{\mathrm{loc}}(0)$ we conclude from this that

$$
\varphi_{t}(w) \leq 2 r_{1} A|\operatorname{Im} w|, w \in V \cap B\left(0, r_{2}\right) .
$$

Since $z_{0} \in V_{t, d} \cap K$ and $0<t \leq t_{0}$ the point $w_{0}:=\gamma(t)+t^{d} z_{0}$ belongs to $V \cap B\left(0, r_{2}\right)$ by the choice of $t_{0}$. Hence the definition of $\varphi_{t}$ and the property 3.2(d) of $H$ imply

$$
\begin{aligned}
2 r_{1} A t^{d}\left|\operatorname{Im} z_{0}\right| & =2 r_{1} A\left|\operatorname{Im} w_{0}\right| \geq \varphi_{t}\left(w_{0}\right) \\
& \geq \frac{\eta t^{d}}{2}\left(u_{t}\left(z_{0}\right)+2 H\left(\frac{z_{0}-\operatorname{Re} z_{0}}{\eta}\right)\right) \geq \frac{\eta t^{d}}{2} u_{t}\left(z_{0}\right) .
\end{aligned}
$$

Hence we get the estimate

$$
u_{t}\left(z_{0}\right) \leq \frac{4 A r_{1}}{\eta}\left|\operatorname{Im} z_{0}\right| \leq A_{0}\left|\operatorname{Im} z_{0}\right|,
$$

which proves $(\gamma)$.

From [7, Theorem 3.7, it follows that for each pure dimensional algebraic variety $V$ in $\mathbb{C}^{n}$ which satisfies $\mathrm{PL}_{\text {loc }}(\xi)$ for $\xi \in V \cap \mathbb{R}^{n}$, the tangent variety $T_{\xi} V$ satisfies $\mathrm{PL}_{\text {loc }}(0)$. Using Lemma 3.4 and basic results from Meise, Taylor, and Vogt [24, Section 4 , we now show that $\mathrm{PL}_{\mathrm{loc}}$ is inherited even by tangent varieties along real simple curves.

3.5. Proposition. Let $V$ be an analytic variety of pure dimension $k$ in $\mathbb{C}^{n}$ which satisfies $0 \in V$. If $V$ satisfies $\mathrm{PL}_{\mathrm{loc}}(0)$, then the following condition is satisfied:

(*) For each simple curve $\gamma$ and each $d \in\left[1, \infty\left[\right.\right.$ the variety $T_{\gamma, d} V$ satisfies $\mathrm{PL}_{\text {loc }}(\xi)$ at each point $\xi \in T_{\gamma, d} V \cap \mathbb{R}^{n}$.

In particular, $T_{0} V$ satisfies $\mathrm{PL}_{\mathrm{loc}}(0)$.

Proof. To prove $(*)$ we will use extremal plurisubharmonic functions which are defined as follows (see Siciak [30], Zeriahi [36], or Meise, Taylor, and Vogt [24]): Let $V$ be an analytic variety in a domain $D$ in $\mathbb{C}^{n}$ and let $E$ be a subset of $D$. Then the extremal plurisubharmonic function $U_{E}(\cdot ; V, D)$ of $E$ relative to $V$ and $D$ is defined as

$$
U_{E}(z ; V, D):=\sup \left\{u(z): u \in \operatorname{PSH}(V), u(\zeta) \leq 1, \zeta \in V \cap D,\left.u\right|_{E \cap V}=0\right\} .
$$

This is Definition 4.1 in [24] with $h \equiv 1$. 
Next fix $\xi \in T_{\gamma, d} V \cap \mathbb{R}^{n}$ and let $D_{0}:=B(\xi, 3)$. It is no restriction to assume that $\gamma$ is defined on $] 0, \alpha$ [ and that $V_{t, d} \cap D_{0}$ is a closed analytic subset of $D_{0}$ for each $t \in] 0, \alpha[$. Then let

$$
D:=B(\xi, 2) \quad \text { and } \quad K:=\overline{B(\xi, 1)},
$$

apply Lemma 3.4 with $D$ and $K$ to get positive numbers $A_{0}$ and $t_{0}$ according to 3.4 , and let

$$
K_{0}:=\left\{x \in \mathbb{R}^{n}:|x-\xi| \leq 2\right\} .
$$

To obtain a suitable estimate of the extremal functions $U_{K_{0}}\left(\cdot ; V_{t, d} \cap D_{0}, D_{0}\right)$ on $K$, fix $0<t \leq t_{0}$, and $u \in \operatorname{PSH}\left(V_{t, d} \cap D_{0}\right)$ and assume that

$$
u(z) \leq 1, z \in V_{t, d} \cap D_{0} \quad \text { and }\left.\quad u\right|_{K_{0} \cap V_{t, d}}=0 .
$$

Then define the family $\left(u_{\tau}\right)_{\tau \in] 0, \alpha[}$ by

$$
\left.u_{\tau}:=0 \text { for } \tau \in\right] 0, \alpha\left[\text { and } \tau \neq t \text { and } u_{t}:=u\right. \text {. }
$$

Obviously, $\left(u_{\tau}\right)_{\tau \in] 0, \alpha[}$ satisfies the hypotheses of Lemma 3.4 and consequently

$$
u(z) \leq A_{0}|\operatorname{Im} z|, \quad z \in V_{t, d} \cap K .
$$

By the definition of the extremal function, this implies

$$
U_{K_{0}}\left(z ; V_{t, d} \cap D_{0}, D_{0}\right) \leq A_{0}|\operatorname{Im} z|, z \in V_{t, d} \cap K, 0<t \leq t_{0} .
$$

Now fix some sequence $\left(t_{j}\right)_{j \in \mathbb{N}}$ in $] 0, \alpha\left[\right.$ with $\lim _{j \rightarrow \infty} t_{j}=0$. By Theorem 2.7(d) the sequence $\left(V_{t_{j}, d} \cap D_{0}\right)_{j \in \mathbb{N}}$ of varieties converges to $T_{\gamma, d} \cap D_{0}$ in the sense of Meise, Taylor, and Vogt [24], Definition 4.3. Hence it follows from [24], Theorem 4.4, that for each regular point $z$ of $T_{\gamma, d} V$ in $B(\xi, 1)$ and each sequence $\left(z_{j}\right)_{j \in \mathbb{N}}$ satisfying $z_{j} \in V_{t_{j}, d}$ and $z_{j} \rightarrow z$, we have

$$
U_{K_{0}}\left(z ; T_{\gamma, d} V \cap D_{0}, D_{0}\right) \leq \liminf _{j \rightarrow \infty} U_{K_{0}}\left(z_{j}, V_{t_{j}, d} \cap D_{0}, D_{0}\right) .
$$

From this estimate and (3.1) it follows that

$$
U_{K_{0}}\left(z ; T_{\gamma, d} V \cap D_{0}, D_{0}\right) \leq A_{0}|\operatorname{Im} z|, z \in\left(T_{\gamma, d} V\right)_{\operatorname{reg}} \cap B(\xi, 1) .
$$

To derive from (3.2) that $T_{\gamma, d} V$ satisfies $\mathrm{PL}_{\text {loc }}(\xi)$ fix $u \in \operatorname{PSH}\left(T_{\gamma, d} V \cap D_{0}\right)$ satisfying

$$
u(z) \leq 1, z \in T_{\gamma, d} V \cap D_{0} \text {, and } u(z) \leq 0, z \in T_{\gamma, d} V \cap B\left(\xi, \frac{5}{2}\right) \cap \mathbb{R}^{n} .
$$

Then the definition of the extremal plurisubharmonic function

$$
U_{K_{0}}\left(\cdot ; T_{\gamma, d} V \cap D_{0}, D_{0}\right)
$$

implies

$$
u(z) \leq U_{K_{0}}\left(z ; T_{\gamma, d} V \cap D_{0}, D_{0}\right), z \in T_{\gamma, d} V \cap D_{0} .
$$

By (3.2), this estimate gives

$$
u(z) \leq A_{0}|\operatorname{Im} z|, z \in\left(T_{\gamma, d} V\right)_{\mathrm{reg}} \cap B(\xi, 1) .
$$

Now our definition of a plurisubharmonic function implies that this estimate holds on $T_{\gamma, d} V \cap B(\xi, 1)$. Hence $u$ satisfies the conditions of Lemma 3.3(b) with $r_{1}=$ $3, r_{2}=5 / 2, r_{3}=1$, and $A=A_{0}$. Consequently, $T_{\gamma, d} V$ satisfies $\mathrm{PL}_{\mathrm{loc}}(\xi)$.

To prove the additional statement, fix $\xi \in \mathbb{R}^{n}$ with $|\xi|=1$ and let $\left.\gamma:=\right] 0,1[\rightarrow$ $\mathbb{R}^{n}, \gamma(t):=t \xi$. Then 2.7(b) implies $T_{\gamma, 1} V=T_{0} V-\xi$. By $(*), T_{\gamma, 1} V$ satisfies $\mathrm{PL}_{\mathrm{loc}}(-\xi)$, hence $T_{0} V$ satisfies $\mathrm{PL}_{\mathrm{loc}}(0)$. 
In order to derive further necessary conditions for $\mathrm{PL}_{\mathrm{loc}}$, we recall the local radial Phragmén-Lindelöf condition $\mathrm{RPL}_{\text {loc }}$ from Meise, Taylor, and Vogt [24, 2.3. It is easy to see that the definition given here is equivalent to the one in [24], 2.3. Further equivalent formulations - analogous to those given for $\mathrm{PL}_{\mathrm{loc}}$ in Lemma 3.3 - can be derived from [8], Lemma 8.

3.6. Definition. For $\xi \in \mathbb{R}^{n}$ and $r_{0}>0$ let $V$ be an analytic variety in $B(\xi, r)$ which contains $\xi$. We say that $V$ satisfies the condition $\operatorname{RPL}_{\text {loc }}(\xi)$ if there exist positive numbers $A$ and $r_{0} \geq r_{1} \geq r_{2}$ such that each $u \in \operatorname{PSH}\left(V \cap B\left(0, r_{1}\right)\right)$ satisfying

$(\alpha) u(z) \leq 1, z \in V \cap B\left(0, r_{1}\right)$ and

( $\beta) u(z) \leq 0, z \in V \cap \mathbb{R}^{n} \cap B\left(0, r_{2}\right)$

also satisfies

$(\gamma)^{\prime} u(z) \leq A|z-\xi|, z \in V \cap B\left(0, r_{1}\right)$.

3.7. Remark. Let $V$ be an analytic variety in some open set in $\mathbb{C}^{n}$. If $V$ satisfies $\mathrm{PL}_{\text {loc }}(\xi)$ at some $\xi \in V \cap \mathbb{R}^{n}$, then Lemma 3.3 implies obviously that $V$ satisfies $\mathrm{RPL}_{\text {loc }}(\xi)$. Hence it follows from Meise, Taylor, and Vogt [24], Lemma 2.8, that $V$ satisfies the dimension condition at $\xi$, which is defined as follows:

3.8. Definition. Let $V$ be an analytic variety in a neighborhood of a point $\xi \epsilon$ $V \cap \mathbb{R}^{n}$. We say that $V$ satisfies the dimension condition at $\xi$ if for each locally irreducible component $W$ of $V$ at $\xi$, the dimension of $W \cap \mathbb{R}^{n}$ as a real analytic variety is equal to the dimension of $W$ at $\xi$ as a complex variety.

In order to state and to prove further necessary conditions for $\mathrm{PL}_{\text {loc }}(\xi)$ we introduce the following notions:

3.9. Definition. Let $V$ be an analytic variety in $\mathbb{C}^{n}$ which is of pure dimension $k \geq 1$ in $\zeta \in V$. A projection $\pi: \mathbb{C}^{n} \rightarrow \mathbb{C}^{n}$ is called noncharacteristic for $V$ at $\zeta$ if its rank is $k$, its image and its kernel are spanned by real vectors, and $T_{\zeta} V \cap \operatorname{ker} \pi=\{0\}$.

Remark. Let $V$ be an analytic variety of pure dimension $k$ in a neighborhood of $\zeta \in \mathbb{C}^{n}$ with $\zeta \in V$. Then there exist noncharacteristic projections for $V$ at $\zeta$.

Proof. We may assume $\zeta=0$. Let $\operatorname{Grass}(n-k, n)$ denote the (complex) Grassmannian, i.e., the manifold of all linear subspaces of $\mathbb{C}^{n}$ of dimension $n-k$, and let $\operatorname{Grass}_{\mathbb{R}}(n-k, n)$ be the real Grassmannian. Then $\operatorname{Grass}_{\mathbb{R}}(n-k, n) \subset \operatorname{Grass}(n-k, n)$ canonically. Note that $L \in \operatorname{Grass}(n-k, n)$ is in $\operatorname{Grass}_{\mathbb{R}}(n-k, n)$ if and only if all its Plücker coordinates are real. Since no nonzero polynomial vanishes on all real points, this implies that the real Grassmannian $\operatorname{Grass}_{\mathbb{R}}(n-k, n)$ is dense in $\operatorname{Grass}(n-k, n)$ in the Zariski topology. On the other hand, it is well known that the linear subspaces of $\mathbb{C}^{n}$ of dimension $n-k$ intersecting $V$ transversally at 0 form an open and dense subset of $\operatorname{Grass}(n-k, n)$ (cf., e.g., 14, 3.8, proof of Corollary 2). Hence there is also $L \in \operatorname{Grass}_{\mathbb{R}}(n-k, n)$ intersecting $V$ transversally at 0 . Any projection $\pi$ whose kernel is the complexification of $L$ and whose image is spanned by real vectors is noncharacteristic for $V$ at 0 .

3.10. Lemma. Let $V$ be an analytic variety in $\mathbb{C}^{n}$ which contains the origin and which is of pure dimension $k \geq 1$. If $\pi: \mathbb{C}^{n} \rightarrow \mathbb{C}^{n}, \pi\left(z^{\prime}, z^{\prime \prime}\right) \in \mathbb{C}^{k} \times \mathbb{C}^{n-k}$ is noncharacteristic for $V$ at 0 , then there exist $C \geq 1$ and $0<r_{0}<1$ such that for $0<r<r_{0}$ we have

$$
V \cap\left(B^{k}(0, r) \times B^{n-k}(0,4 C r)\right) \subset V \cap\left(B^{k}(0, r) \times B^{n-k}(0,3 C r)\right) .
$$


Proof. At first we claim that there is $C \geq 1$ such that

$$
\left|z^{\prime \prime}\right| \leq C\left|z^{\prime}\right| \text { for all }\left(z^{\prime}, z^{\prime \prime}\right) \in T_{0} V \text {. }
$$

To prove this let

$$
\delta:=\inf \left\{|\pi(w)|: w \in T_{0} V,\left|w^{\prime \prime}\right|=1\right\} .
$$

If we assume that $\delta=0$, then there exists a sequence $\left(w_{j}\right)_{j \in \mathbb{N}}$ in $T_{0} V, w_{j}=\left(w_{j}^{\prime}, w_{j}^{\prime \prime}\right)$ satisfying $\lim _{j \rightarrow \infty} w_{j}^{\prime}=0$ and $\left|w_{j}^{\prime \prime}\right|=1$ for all $j \in \mathbb{N}$. Passing to a subsequence we find $\left(0, w_{0}^{\prime \prime}\right) \in T_{0} V$ with $\left|w_{0}^{\prime \prime}\right|=1$, in contradiction to $\pi$ being noncharacteristic for $V$ at the origin. Hence we have $\delta>0$. From this and a standard scaling argument we get (3.3) with $C=1 / \delta$. Of course, we may assume $C \geq 1$.

Next let $V_{t}:=\frac{1}{t} V$ for $0<t<1$ and define

$$
K:=\overline{B^{k}(0,2)} \times \overline{B^{n-k}(0,4 C)} \text { and } G:=\left(T_{0} V \cap K\right)+B^{k}(0,1) \times B^{n-k}(0,1) .
$$

By 9, 3.13, and Lemma 2.8 there exists $0<\tau<1$ such that

$$
\left.V_{t} \cap K \subset G \text { for all } t \in\right] 0, \tau[\text {. }
$$

Now fix $0<t<\tau$ and $z \in V_{t} \cap B^{k}(0,1) \times B^{n-k}(0,4 C)$. Then $z=\left(z^{\prime}, z^{\prime \prime}\right)$ belongs to $V_{t} \cap K$ and hence to $G$ by (3.4). This implies the existence of $\zeta \in T_{0} V \cap K$ such that

$$
\left|z^{\prime}-\zeta^{\prime}\right|<1 \text { and }\left|z^{\prime \prime}-\zeta^{\prime \prime}\right|<1
$$

From this and (3.3) we get $\left|\zeta^{\prime}\right|<2$ and

$$
\left|z^{\prime \prime}\right| \leq\left|z^{\prime \prime}-\zeta^{\prime \prime}\right|+\left|\zeta^{\prime \prime}\right|<1+C\left|\zeta^{\prime}\right|<1+2 C \leq 3 C .
$$

Thus we have shown

$$
V_{t} \cap\left(B^{k}(0,1) \times B^{n-k}(0,4 C)\right) \subset V_{t} \cap\left(B^{k}(0,1) \times B^{n-k}(0,3 C)\right), 0<t<t .
$$

Since $V_{t}=\frac{1}{t} V$, this implies the assertion of the lemma.

3.11. Definition. Let $V$ be an analytic variety of pure dimension $k$ in $\mathbb{C}^{n}$ which contains the origin, let $\gamma$ be a real simple curve, let $d \geq 1$, and let $\zeta \in T_{\gamma, d} V \cap \mathbb{R}^{n}$. We say that $V$ is $(\gamma, d)$-hyperbolic at $\zeta$ with respect to a projection $\pi: \mathbb{C}^{n} \rightarrow \mathbb{C}^{n}$ which is noncharacteristic for $T_{\gamma, d} V$ at $\zeta$ if there exist a zero neighborhood $U$ in $\mathbb{C}^{n}$ and $r>0$ such that $z \in V \cap \Gamma(\gamma, d, \zeta+U, r)$ is real whenever $\pi(z)$ is real. $V$ is called $(\gamma, d)$-hyperbolic at $\zeta$ if it is $(\gamma, d)$-hyperbolic at $\zeta$ with respect to some projection $\pi$ as above.

3.12. Proposition. Let $V \subset \mathbb{C}^{n}$ be a pure $k$-dimensional analytic variety in $\mathbb{C}^{n}$ which contains the origin and satisfies $\mathrm{PL}_{\mathrm{loc}}(0)$, and let $d \geq 1$. Then for each real simple curve $\gamma:] 0, \alpha\left[\rightarrow \mathbb{R}^{n}\right.$, each $\xi \in\left(T_{\gamma, d} V\right)_{\text {reg }} \cap \mathbb{R}^{n}$, and each projection $\pi: \mathbb{C}^{n} \rightarrow \mathbb{C}^{n}$ which is noncharacteristic for $T_{\gamma, d} V$ at $\xi, V$ is $(\gamma, d)$-hyperbolic at $\xi$ with respect to $\pi$.

Proof. After a real linear change of variables we may assume $\mathbb{C}^{n}=\mathbb{C}^{k} \times \mathbb{C}^{n-k}$ and $\pi\left(z^{\prime}, z^{\prime \prime}\right)=\left(z^{\prime}, 0\right)$ for $z=\left(z^{\prime}, z^{\prime \prime}\right) \in \mathbb{C}^{k} \times \mathbb{C}^{n-k}$. Since $\pi$ is noncharacteristic for $T_{\gamma, d} V$ in $\xi$ we get from Lemma 3.10 the existence of numbers $\varepsilon^{\prime}>0$ and $0<\varepsilon_{1}^{\prime \prime}<\varepsilon^{\prime \prime}$ such that with $\xi=\left(\xi^{\prime}, \xi^{\prime \prime}\right)$ we have

$$
B^{k}\left(\xi^{\prime}, \varepsilon^{\prime}\right) \times B^{n-k}\left(\xi^{\prime \prime}, \varepsilon^{\prime \prime}\right) \cap T_{\gamma, d} V \subset B^{k}\left(\xi^{\prime}, \varepsilon^{\prime}\right) \times B^{n-k}\left(\xi^{\prime \prime}, \varepsilon_{1}^{\prime \prime}\right) .
$$

Since $\xi$ is a regular point of $T_{\gamma, d} V$, we may assume that $\varepsilon^{\prime}$ is chosen so that there exists a holomorphic map $g: B^{k}\left(\xi^{\prime}, \varepsilon^{\prime}\right) \rightarrow B^{n-k}\left(\xi^{\prime \prime}, \varepsilon_{1}^{\prime \prime}\right)$ such that

$$
T_{\gamma, d} V \cap B^{k}\left(\xi^{\prime}, \varepsilon^{\prime}\right) \times B^{n-k}\left(\xi^{\prime \prime}, \varepsilon_{1}^{\prime \prime}\right)=\left\{(w, g(w)): w \in B^{k}\left(\xi^{\prime}, \varepsilon^{\prime}\right)\right\} .
$$


Next fix $0<\varepsilon_{1}^{\prime}<\varepsilon_{2}^{\prime}<\varepsilon^{\prime}$ and $\varepsilon_{1}^{\prime \prime}<\varepsilon_{2}^{\prime \prime}<\varepsilon^{\prime \prime}$ and let

$$
D:=B^{k}\left(\xi^{\prime}, \varepsilon_{2}^{\prime}\right) \times B^{n-k}\left(\xi^{\prime \prime}, \varepsilon_{2}^{\prime \prime}\right), Q:=\bar{D} \text {, and } K:=\overline{B^{k}\left(\xi^{\prime}, \varepsilon_{1}^{\prime}\right) \times B^{n-k}\left(\xi^{\prime \prime}, \varepsilon_{1}^{\prime \prime}\right)} \text {. }
$$

Since $K$ is a compact subset of $D$ we can apply Lemma 3.4 to these sets and $\left(V_{t, d}\right)_{t \in] 0, \alpha[}$ to get $A_{0}>0$ and $0<t_{0}<\alpha$ so that the conclusion of that lemma holds. Furthermore, the differentiability of $g$ on $B^{k}\left(\xi^{\prime}, \varepsilon^{\prime}\right)$ implies the existence of $C>0$ such that

$$
|g(u)-g(w)| \leq C|u-w|, \quad u, w \in \overline{B^{k}\left(\xi^{\prime}, \varepsilon_{2}^{\prime}\right)} .
$$

Now choose $0<\eta \leq\left(1+A_{0}\right)^{-1}(1+C)^{-1}$ so small that

$$
Q+B^{n}(0, \eta) \subset B^{k}\left(\xi^{\prime}, \varepsilon^{\prime}\right) \times B^{n-k}\left(\xi^{\prime \prime}, \varepsilon^{\prime \prime}\right) .
$$

Then note that by Lemma 2.8 there exists $0<t_{1} \leq t_{0}$ such that

$$
V_{t, d} \cap Q \subset T_{\gamma, d} V \cap\left(Q+B^{n}(0, \eta)\right), \quad 0<t<t_{1} .
$$

In order to apply Lemma 3.4 define the family $\left(u_{t}\right)_{t \in] 0, \alpha[}$ of functions $u_{t} \in$ $\operatorname{PSH}\left(V_{t, d} \cap D\right)$ as follows: If $t_{1} \leq t<\alpha$ then $u_{t}:=0$ and if $0<t<t_{1}$ then

$$
u_{t}(w, s):=\left(A_{0}+1\right)|\operatorname{Im}(s-g(w))|, \quad(w, s) \in V_{t, d} \cap D .
$$

To show that $\left(u_{t}\right)_{t \in] 0, \alpha[}$ satisfies the condition 3.4( $\left.\alpha\right)$, note first that

$$
T_{\gamma, d} V \cap Q=\left\{(u, g(u)):\left|\xi^{\prime}-u\right| \leq \varepsilon_{2}^{\prime}\right\} .
$$

Then fix $0<t<t_{1}$ and $(w, s) \in V_{t, d} \cap D \subset V_{t, d} \cap Q$. By the choice of $t_{1}$ there exists $(u, g(u)) \in T_{\gamma, d} V \cap Q$ such that

$$
|(w, s)-(u, g(u))|<\eta .
$$

From this and our choices above we get

$$
|s-g(w)| \leq|s-g(u)|+|g(u)-g(w)| \leq \eta+C|u-w| \leq \eta(C+1) \leq \frac{1}{A_{0}+1} .
$$

Obviously, this implies

$$
u_{t}(w, s)=\left(A_{0}+1\right)|\operatorname{Im}(s-g(w))| \leq 1,(w, s) \in V_{t, d} \cap D, 0<t<t_{1} .
$$

Hence the estimate $3.4(\alpha)$ holds.

To show that $3.4(\beta)$ also holds, note first that $T_{\gamma, d} V$ satisfies $\mathrm{PL}_{\text {loc }}(\xi)$ by Proposition 3.5. Hence $T_{\gamma, d} V$ satisfies the dimension condition at $\xi$, by Remark 3.7. Since $\xi$ is a regular point of $T_{\gamma, d} V$, this implies that $g(w)$ is real for real $w$ and hence

$$
u_{t}(w, s) \leq 0 \text { if }(w, s) \in V_{t, d} \cap D \cap \mathbb{R}^{n}, 0<t<t_{1} .
$$

Thus $\left(u_{t}\right)_{t \in] 0, \alpha[}$ also satisfies condition $3.4(\beta)$. By Lemma 3.4, $\left(u_{t}\right)_{t \in] 0, \alpha[}$ also satisfies $(\gamma)$, i.e.,

$$
u_{t}(z) \leq A_{0}|\operatorname{Im} z|, \quad z \in V_{t, d} \cap K, \quad 0<t<t_{1} .
$$

Now fix $(w, s) \in V_{t, d} \cap K$ and assume that $\pi(w, s) \in \mathbb{R}^{n}$. Then $w \in \mathbb{R}^{k}$ implies $g(w) \in \mathbb{R}^{n-k}$ and consequently

$$
\left(A_{0}+1\right)|\operatorname{Im} s|=\left(A_{0}+1\right)|\operatorname{Im}(s-g(w))|=u_{t}(w, s) \leq A_{0}|\operatorname{Im} s| .
$$

From this we conclude that $\operatorname{Im} s=0$, i.e., $(w, s) \in \mathbb{R}^{n}$.

Let $U:=B^{k}\left(0, \varepsilon_{1}^{\prime}\right) \times B^{n-k}\left(0, \varepsilon_{1}^{\prime \prime}\right)$ and assume that for

$$
(\zeta, \sigma) \in V \cap \Gamma\left(\gamma, d, \xi+U, t_{1}\right),
$$

$\pi(\zeta, \sigma)=\zeta$ is real. Then there exist $t \in] 0, t_{1}[$ and $(w, s) \in \xi+U$ such that $(\zeta, \sigma)=\gamma(t)+t^{d}(w, s)$. Hence $(w, s) \in V_{t, d} \cap K$ and $w \in \mathbb{R}^{n}$. By the above, this 
implies $s \in \mathbb{R}^{n-k}$ and consequently $(\zeta, \sigma) \in \mathbb{R}^{n}$. Thus $V$ is $(\gamma, d)$-hyperbolic at $\xi$ with respect to $\pi$.

Combining Proposition 3.5 and Proposition 3.12, we have proved the following necessary condition for $\mathrm{PL}_{\mathrm{loc}}$.

3.13. Theorem. Let $V$ be an analytic variety of pure dimension $k \geq 1$ in $\mathbb{C}^{n}$ which contains the origin. If $V$ satisfies $\mathrm{PL}_{\mathrm{loc}}(0)$, then for each real simple curve $\gamma$ in $\mathbb{C}^{n}$ and for each $d \geq 1$ for which $T_{\gamma, d} V \neq \emptyset$, the following two conditions hold:

(a) $T_{\gamma, d} V$ satisfies $\mathrm{PL}_{\mathrm{loc}}(\zeta)$ for each $\zeta \in T_{\gamma, d} V \cap \mathbb{R}^{n}$.

(b) $V$ is $(\gamma, d)$-hyperbolic at every real point of $T_{\gamma, d} V$ which is regular.

3.14. Definition. If $V$ satisfies the conditions of Theorem 3.13, then we will say that $V$ is hyperbolic in conoids at the origin.

For analytic curves in $\mathbb{C}^{n}$ and for surfaces in $\mathbb{C}^{3}$ the conditions in Theorem 3.13 are actually characterizing. To prove the first statement together with another equivalent formulation, we recall the following definition from Andersson [1], resp. Hörmander [17, Definition 6.4.

3.15. Definition. Let $V$ be an analytic variety of pure dimension $k$ in $\mathbb{C}^{n}$ and let $\xi \in V \cap \mathbb{R}^{n}$. We say that $V$ is locally hyperbolic at $\xi$ if there are a neighborhood $U$ of $\xi$ and a projection $\pi: \mathbb{C}^{n} \rightarrow \mathbb{C}^{n}$ which is noncharacteristic for $V$ at $\xi$ such that $z \in V \cap U$ is real whenever $\pi(z)$ is real.

For $n=2$ the following result is already contained in Braun [5] and Zampieri 33.

3.16. Proposition. Let $V \subset \mathbb{C}^{n}$ be an analytic variety of pure dimension 1 which contains the origin. Then the following assertions are equivalent:

(a) $V$ satisfies $\mathrm{PL}_{\text {loc }}(0)$.

(b) There exist a real linear change of variables as well as positive numbers $\rho$ and $\delta, k \in \mathbb{N}$ and holomorphic maps $g_{j}: B^{1}(0, \rho) \rightarrow \mathbb{C}^{n-1}$ satisfying $g_{j}\left(B^{1}(0, \rho) \cap \mathbb{R}\right) \subset \mathbb{R}^{n-1}, 1 \leq j \leq k$, such that

$$
V \cap\left(B^{1}(0, \rho) \times B^{n-1}(0, \delta)\right)=\bigcup_{j=1}^{k}\left\{\left(z_{1}, g_{j}\left(z_{1}\right)\right): z_{1} \in B^{1}(0, \rho)\right\} .
$$

(c) $V$ is locally hyperbolic at 0.

Proof. (a) $\Rightarrow\left(\right.$ b): Since $V$ satisfies $\mathrm{PL}_{\mathrm{loc}}(0)$ if and only if each irreducible component of $V$ has this property, assume first that $V$ is locally irreducible in 0 . Then there exists $a \in \mathbb{C}^{n}$ such that $T_{0} V=\mathbb{C} \cdot a$, by Chirka [14, Proposition 8.1. Since $T_{0} V$ satisfies $\mathrm{PL}_{\mathrm{loc}}(0)$ by Proposition 3.5 and consequently the dimension condition, it follows that $a=\lambda b$ for some $b \in \mathbb{R}^{n}, \lambda \in \mathbb{C}$. Performing a real linear change of variables, we may assume that $b=e_{1}$, the first canonical basis vector of $\mathbb{C}^{n}$. Then $\pi: \mathbb{C}^{n} \rightarrow \mathbb{C}^{n}, \quad \pi(z)=\left(z_{1}, 0, \cdots, 0\right)$ is a noncharacteristic projection for $V$. Moreover, it follows from Chirka [14, Proposition 8.1, that there are $\varepsilon_{1}, \varepsilon_{2}>0$ such that for $U:=B^{1}\left(0, \varepsilon_{1}\right) \times B^{n-1}\left(0, \varepsilon_{2}\right)$ we have

$$
V \cap U=\left\{\left(z^{q}, \sum_{j>q} a_{j} z^{j}\right), \quad|z|<\varepsilon_{1}\right\},
$$


where $a_{j} \in \mathbb{C}^{n-1}$ for $j>q$. For $\gamma_{ \pm}: t \mapsto \pm e_{1} t, t>0$, we get by (3.5) from Theorem 2.7(b) that $T_{\gamma_{ \pm}, 1} V=T_{0} V \mp e_{1}$. Since $\pm e_{1}$ is a regular point of $T_{0} V, 0$ is a regular point of $T_{\gamma_{ \pm}, 1} V$. By Proposition 3.12 this implies that $V$ is $\left(\gamma_{ \pm}, 1\right)$-hyperbolic in the cones $\Gamma\left(\gamma_{ \pm}, 1, U^{ \pm}, r\right)$ for the projection $\pi$ defined above, some $r>0$, and some zero neighborhoods $U^{ \pm}$in $\mathbb{C}^{n}$. Consequently we have

$$
\left.g(z):=\sum_{j>q} a_{j} z^{j} \text { is real whenever } z^{q} \in\right]-r, r[.
$$

In particular, $g(z)$ is real for real $z$ near the origin and hence $a_{j} \in \mathbb{R}^{n-1}$ for all $j>q$. Now this and (3.6) imply $a_{j}=0$ whenever $j$ is not a multiple of $q$. Therefore we may assume $q=1$ and obtain the assertion (b) if $V$ is irreducible.

If $V$ is reducible, we decompose $V=\bigcup_{j=1}^{k} V_{j}$ into its irreducible branches. Then the hypothesis in (a) implies that we have $T_{0} V=\bigcup_{\nu=1}^{k} \mathbb{C} b_{\nu}$, where $b_{\nu} \in \mathbb{R}^{n} \backslash\{0\}$ for $1 \leq \nu \leq k$. Now choose a real hyperplane $H$ in $\mathbb{R}^{n}$ satisfying $b_{\nu} \notin H, 1 \leq \nu \leq k$, and $\eta_{1} \in \mathbb{R}^{n}, \eta_{1} \notin H$. If $H=\operatorname{span}\left\{\eta_{2} \cdots, \eta_{n}\right\}$, then it follows easily from the considerations in the first part of the proof that (b) holds in the coordinates induced by the basis $\left(\eta_{1}, \cdots, \eta_{n}\right)$.

(b) $\Rightarrow(\mathrm{c})$ : Denote by $\pi: \mathbb{C}^{n} \rightarrow \mathbb{C}^{n}$ the map which is defined by $\pi(z):=$ $\left(z_{1}, 0, \cdots, 0\right)$ in the coordinates existing by (b) and let $U:=B^{1}(0, \rho) \times B^{n-1}(0, \delta)$. Then it is an obvious consequence of (b) that $V$ is locally hyperbolic in 0 .

(c) $\Rightarrow$ (a): It is no restriction to assume that the projection $\pi$ which exists by (c) has the form $\pi(z)=\left(z_{1}, 0, \cdots, 0\right)$. Since $\pi$ is noncharacteristic, we have $T_{0} V \cap \operatorname{ker} \pi=\{0\}$. Hence we can choose $\rho>0$ and $\delta>0$ so that

$$
V \cap\left\{\left(z_{1}, z^{\prime}\right) \in \mathbb{C}^{n}:\left|z_{1}\right| \leq \rho,\left|z^{\prime}\right|=\delta\right\}=\emptyset .
$$

To prove that $V$ satisfies $\mathrm{PL}_{\text {loc }}(0)$ let $r_{1}:=\min (\rho, \delta)$ and fix $u \in \operatorname{PSH}\left(V \cap B\left(0, r_{1}\right)\right)$ satisfying $(\alpha)$ and $(\beta)$ of Definition 3.1. Then define $\varphi: B^{1}\left(0, r_{1}\right) \rightarrow[-\infty, \infty[$ by

$$
\varphi(\lambda):=\max \{u(z): z \in V, \pi(z)=(\lambda, 0, \cdots, 0)\} .
$$

Then $\varphi$ is subharmonic in $B_{ \pm}:=\left\{\lambda \in \mathbb{C}:|\lambda|<r_{1}, \operatorname{Im} \pm \lambda>0\right\}$ obviously, $\varphi(\lambda) \leq 1$ for $\lambda \in B_{ \pm}$. Since $z$ is real when $\pi(z)$ is real, it follows that $\varphi(\lambda) \leq 0$ for $\lambda \in$ $\partial B_{ \pm} \cap \mathbb{R}$. Hence a classical estimate for the harmonic measure of the half disk (see Nevanlinna 28, 38) implies the existence of $A>0$, depending only on $r_{1}$, such that

$$
\varphi(\lambda) \leq A|\operatorname{Im} \lambda|, \quad \lambda \in B^{1}\left(0, \frac{r_{1}}{2}\right) .
$$

By the definition of $\varphi$ this implies

$$
u(z) \leq A\left|\operatorname{Im} z_{1}\right| \leq A|\operatorname{Im} z|, \quad z \in V \cap B^{n}\left(0, \frac{r_{1}}{2}\right) .
$$

Hence $V$ satisfies $\mathrm{PL}_{\text {loc }}(0)$.

Using Proposition 3.16 we can now reformulate some of the conditions in Theorem 3.13 for surfaces in $\mathbb{C}^{3}$, using the following lemma.

3.17. Lemma. Let $V \subset \mathbb{C}^{3}$ be an analytic surface which contains the origin.

(a) If $V$ satisfies $\mathrm{PL}_{\mathrm{loc}}(0)$, then for each real simple curve $\gamma$ and for each $d>1$ the limit variety $T_{\gamma, d} V$ is locally hyperbolic at each of its real points.

(b) If $T_{0} V$ satisfies $\mathrm{PL}_{\mathrm{loc}}(0)$, then $T_{0} V$ is locally hyperbolic at each $\xi \in T_{0} V \cap$ $\mathbb{R}^{3}, \xi \neq 0$. 
Proof. (a) By Theorem 2.7(c) we can choose coordinates $\mathfrak{X}$ in $\mathbb{C}^{3}$ so that

$$
T_{\gamma, d} V=\mathbb{C} \times C_{\gamma, d, \mathfrak{x}},
$$

where $C_{\gamma, d, \mathfrak{X}}$ is an algebraic curve in $\mathbb{C}^{2}$. Since $T_{\gamma, d} V$ satisfies $\mathrm{PL}_{\text {loc }}(\xi)$ for each

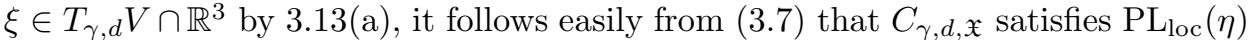
for each $\eta \in C_{\gamma, d, \mathfrak{X}} \cap \mathbb{R}^{2}$. By Proposition 3.16, this property is equivalent to $C_{\gamma, d, \mathfrak{X}}$ being locally hyperbolic at each $\eta \in C_{\gamma, d, \mathfrak{x}} \cap \mathbb{R}^{2}$. From this and (3.7) we get (a).

(b) Since $T_{0} V$ is a homogeneous variety, it suffices to prove (b) for all $\xi \in$ $T_{0} V \cap \mathbb{R}^{3},|\xi|=1$. Fix such a $\xi$ and let $\gamma: t \mapsto t \xi, t>0$. Then Theorem 2.7(b) implies $T_{\gamma, 1} V=T_{0} V-\xi$ and hence $0 \in T_{\gamma, 1} V$. By Proposition 3.5, $T_{\gamma, 1} V$ satisfies $\mathrm{PL}_{\text {loc }}(0)$; consequently $T_{0} V$ satisfies $\mathrm{PL}_{\mathrm{loc}}(\xi)$. By Braun [5], Corollary 12, this implies that $T_{0} V$ is locally hyperbolic at $\xi$.

Remark. Note that in Lemma 3.17(b) $T_{0} V$ need not be locally hyperbolic at the origin, as the example

$$
V:=\left\{(x, y, z) \in \mathbb{C}^{3}: x^{3}+y^{3}+z^{3}=0\right\}
$$

shows. Here $V=T_{0} V$ satisfies $\mathrm{PL}_{\mathrm{loc}}(0)$ but $V$ is not locally hyperbolic at zero.

The following lemma provides a rather coarse but useful necessary condition for $\mathrm{PL}_{\mathrm{loc}}(0)$.

3.18. Lemma. Let $V$ be an analytic variety in $B^{n}\left(0, r_{0}\right), r_{0}>0$, which contains the origin. If $V$ satisfies the dimension condition at 0 , then the vanishing ideal

$$
I(V):=\left\{f \in \mathcal{O}(0):\left.f\right|_{V}=0\right\}
$$

is generated by holomorphic generators having real Taylor coefficients.

Proof. Fix $f \in I(V)$ and choose $r_{1}<r_{0}$ such that $\left.f\right|_{V \cap B\left(0, r_{1}\right)} \equiv 0$ and

$$
f(z)=\sum_{|\alpha|>0} a_{\alpha} z^{\alpha}, \quad z \in B^{n}\left(0, r_{1}\right) .
$$

Let $u_{\alpha}:=\operatorname{Re}\left(a_{\alpha}\right), v_{\alpha}:=\operatorname{Im}\left(a_{\alpha}\right)$ and define

$$
f_{R}(z):=\sum_{|\alpha|>0} u_{\alpha} z^{\alpha}, \quad f_{I}(z):=\sum_{|\alpha|>0} v_{\alpha} z^{\alpha} .
$$

Then $f$ and $f_{R}+i f_{I}$ coincide on $\mathbb{R}^{n} \cap B\left(0, r_{1}\right)$, hence $f_{R}$ and $f_{I}$ vanish on $V \cap$ $\mathbb{R}^{n} \cap B\left(0, r_{1}\right)$. Since $V$ satisfies the dimension condition, this implies that $f_{R}$ and $f_{I}$ belong to $I(V)$.

Remark. It follows from Cartan [13], Propositions 11 and 12 that $V \cap \mathbb{R}^{n}$ is realanalytic coherent if $V$ satisfies the dimension condition at each real point. In particular, Lemma 3.18 is a variant of [13], Proposition 8.

We conclude this section with two lemmas concerning $(\gamma, d)$-hyperbolicity that will be used subsequently.

3.19. Lemma. Let $V$ be an analytic variety of pure dimension $k$ in $\mathbb{C}^{n}$ which contains the origin. Let $\gamma$ be a real simple curve in $\mathbb{C}^{n}, d \geq 1$, and $\kappa \in\left(T_{\gamma, d} V\right)_{\mathrm{reg}} \cap$ $\mathbb{R}^{n}$. If $V$ is $(\gamma, d)$-hyperbolic at $\kappa$, then $V$ is $(\gamma, d)$-hyperbolic at $\kappa$ with respect to each projection $\pi$ which is noncharacteristic for $T_{\gamma, d} V$ at $\kappa$.

The proof of Lemma 3.19 is given in Section 5 following Lemma 5.7 since it requires results and a definition which we provide only in that section. 
To formulate a lemma which will be used in Section 5 for the proof that $\mathrm{PL}_{\text {loc }}(0)$ can be determined by investigating a finite number of conditions we introduce some notation:

Let $V$ be an analytic surface in $\mathbb{C}^{3}$ which contains the origin, let $\gamma$ be a real simple curve in $\mathbb{C}^{3}$, and let $d>1$ be given. The argument for $d=1$ is very similar and will be sketched below.

Let $\xi_{0}$ denote the tangent to $\gamma$ at the origin and choose a basis $\left(e_{1}, e_{2}, e_{3}\right)$ of $\mathbb{R}^{3}$ such that $e_{1}=\xi_{0}$, such that $e_{2}, e_{3} \notin T_{0} V$, and such that $e_{2}$ and $e_{3}$ are noncharacteristic for the algebraic surface $T_{\gamma, d} V \subset \mathbb{C}^{3}$. It is no restriction to assume that $\left(e_{1}, e_{2}, e_{3}\right)$ is the standard basis of $\mathbb{R}^{3}$. Denote by $\pi_{2}$ and $\pi_{3}$ the projections $\pi_{2}\left(z_{1}, z_{2}, z_{3}\right)=\left(z_{1}, z_{3}\right)$ and $\pi_{3}\left(z_{1}, z_{2}, z_{3}\right)=\left(z_{1}, z_{2}\right)$, respectively, and by $B_{j}$ the branch locus of $\pi_{j}: V \rightarrow \pi_{j}(V), j=2,3$, in the sense of Chirka 14, 2.7 (see also Definition 4.2 below). Note that by Theorem 2.7(c) there is an algebraic curve $W$ in $\mathbb{C}^{2}$ such that $T_{\gamma, d} V=\mathbb{C} \times W$. Let $W_{0}:=\{0\} \times W$ and define

$$
M:=\left(T_{\gamma, d} B_{2} \cup T_{\gamma, d} B_{3}\right) \cap\left(T_{\gamma, d} V\right)_{\text {reg }} \cap\left(\{0\} \times \mathbb{R}^{2}\right) .
$$

Note that this implies $M \subset\left(W_{0}\right)_{\text {reg. }}$. Let $F$ be a set which contains exactly one point in each connected component of $\left(W_{0}\right)_{\text {reg }} \cap \mathbb{R}^{3} \backslash M$ and set

$$
\mathcal{F}:=F \cup M \text {. }
$$

For the case $d=1$, choose a basis $\left(e_{1}, e_{2}, e_{3}\right)$ of $\mathbb{R}^{3}$ such that $e_{1}, e_{2}$, and $e_{3}$ are noncharacteristic for $T_{\gamma, 1} V$ and assume again that $\left(e_{1}, e_{2}, e_{3}\right)$ is the standard basis of $\mathbb{R}^{3}$. Let $\pi_{2}$ and $\pi_{3}$ be as before and define $\pi_{1}\left(z_{1}, z_{2}, z_{3}\right)=\left(z_{2}, z_{3}\right)$. Note that $T_{\gamma, 1} V=T_{0} V-\xi_{0}$ by Theorem 2.7(b). Finally, set

$$
M:=\left(T_{\gamma, 1} B_{1} \cup T_{\gamma, 1} B_{2} \cup T_{\gamma, 1} B_{3}\right) \cap\left(T_{\gamma, 1} V\right)_{\text {reg }} \cap\left(S^{2}-\xi_{0}\right)
$$

and define $F$ and $\mathcal{F}$ as before.

Now the following lemma holds:

3.20. Lemma. Under the hypotheses stated above, $\mathcal{F}$ is a finite set, and $V$ is $(\gamma, d)$ hyperbolic at each $\zeta \in\left(T_{\gamma, d} V\right)_{\text {reg }} \cap \mathbb{R}^{3}$ if and only if $V$ is $(\gamma, d)$-hyperbolic at each $\zeta \in \mathcal{F}$.

Proof. The proof will only be given for $d>1$, the other case being completely analogous. For $j=2,3$ the limit variety $T_{\gamma, d} B_{j}$ is the union of a finite number of lines parallel to $e_{1}$ by Theorem 2.7(c). Hence $M$ is finite. This shows in particular that $\left(W_{0}\right)_{\text {reg }} \cap \mathbb{R}^{3} \backslash M$ is semi-algebraic, thus it has only a finite number of connected components by Bochnak, Coste, and Roy 3, Théorème 2.4.4. Hence $\mathcal{F}$ is finite.

Now fix a connected component $G$ of $\left(W_{0}\right)_{\text {reg }} \cap \mathbb{R}^{3} \backslash M$. By the choice of $F$ there is exactly one point, say $\zeta$, in $F \cap G$. Next note that by the definition of $(\gamma, d)$-hyperbolicity, the set

$$
G_{+}:=\{\eta \in G: V \text { is }(\gamma, d) \text {-hyperbolic at } \eta\}
$$

is open in $G$. We will show that

$$
G_{-}:=\{\eta \in G: V \text { is not }(\gamma, d) \text {-hyperbolic at } \eta\}
$$

is also open in $G$. Then it follows that $V$ is $(\gamma, d)$-hyperbolic at each $\eta \in G$ if and only if $V$ is $(\gamma, d)$-hyperbolic at $\zeta$.

To show that $G_{-}$is open, fix $\eta \in G_{-}$. Then $\eta$ is a regular point of $W_{0}$. Hence there exists a neighborhood $U$ of $\eta$ such that at least one of the projections $\pi_{2}$ 
and $\pi_{3}$ is noncharacteristic for each $\kappa \in U \cap W_{0} \cap \mathbb{R}^{3}$. Assume it is $\pi_{2}$. By the choice of $G$, we may assume that $U$ is so small that $\bar{U} \cap T_{\gamma, d} B_{2}=\emptyset$. We claim that this implies the existence of $r>0$ such that $\Gamma:=\Gamma(\gamma, d, U, r)$ satisfies $\Gamma \cap B_{2}=\emptyset$. To show this, assume for contradiction that there are sequences $\left(t_{n}\right)_{n \in \mathbb{N}}$ of positive numbers and $\left(u_{n}\right)_{n \in \mathbb{N}}$ in $U$ with $\gamma\left(t_{n}\right)+t_{n}^{d} u_{n} \in B_{2}$. Then $\left(u_{n}\right)_{n \in \mathbb{N}}$ admits an accumulation point $u \in \bar{U}$. On the other hand, Definition 2.6 implies $u \in T_{\gamma, d} B_{2}$. This is a contradiction.

Since $\pi_{2}$ is noncharacteristic for $T_{\gamma, d} V$ at 0 , an application of Lemmas 2.8 and 3.10 as in the proof of Proposition 3.12 yields a polydisk $U_{1} \subset U$ and $0<r_{1} \leq r$ and hence a conoid $\Gamma_{1}:=\Gamma\left(\gamma, d, U_{1}, r_{1}\right)$ such that $\pi_{2}: V \cap \Gamma_{1} \rightarrow \pi_{2}\left(\Gamma_{1}\right)$ is an analytic cover, which is unramified by the argument of the last paragraph. Hence $V \cap \Gamma_{1}=$ $\bigcup_{l=1}^{L} W_{l}$, where the $W_{l}$ are mutually disjoint and the maps $u_{l}:=\left.\pi_{2}\right|_{W_{l}}: W_{l} \rightarrow$ $\pi_{2}\left(\Gamma_{1}\right)$ are biholomorphic. Since $V$ is not $(\gamma, d)$-hyperbolic at $\eta$, there is a sequence $\left(\eta_{j}\right)_{j \in \mathbb{N}}$ in $V \backslash \mathbb{R}^{3}$ such that $\pi_{2}\left(\eta_{j}\right) \in \mathbb{R}^{3}$ for each $j$ and such that

$$
\lim _{j \rightarrow \infty} t_{j}^{-d}\left(\eta_{j}-\gamma\left(t_{j}\right)\right)=\eta
$$

where $t_{j}$ is the first component of $\eta_{j}$. We may assume without restriction that all $\eta_{j}$ are in $W_{1}$. Then not all coefficients of the Taylor series expansion of the third component of $u_{1}^{-1}$ are real. Hence $V$ is $(\gamma, d)$-hyperbolic at no point in $T_{\gamma, d} W_{1} \cap \mathbb{R}^{3}$. Since the latter is a neighborhood of $\eta$ in $G$, the claim is shown.

\section{Critical levels}

To show that the necessary conditions in Theorem 3.13 are actually sufficient for $\mathrm{PL}_{\mathrm{loc}}(0)$ for analytic surfaces $V$ in $\mathbb{C}^{3}$, we have to analyze very carefully the branching behavior of $V$ near the origin with respect to various coordinate choices. This analysis is prepared in the present section by the concept of critical levels.

4.1. Notation. Let $V$ be an analytic surface in $\mathbb{C}^{3}$ which contains the origin and denote by $V_{1}, \ldots, V_{m}$, the irreducible components of the germ $[V]_{0}$ of $V$ at the origin. Choose $r>0$ so small that $V_{j} \cap B(0, r)$ is the zero set of a holomorphic function $f_{j}$ which is an irreducible element of the ring of holomorphic functions on $B(0, r)$ for $1 \leq j \leq m$. Then $f:=\prod_{j=1}^{m} f_{j}$ is a holomorphic function on $B(0, r)$ which is square-free and has $V \cap B(0, r)$ as its zero set.

4.2. Definition. For $V$ and $f$ as in Notation 4.1 and $\xi \in \mathbb{R}^{3} \backslash T_{0} V$ we define the branch locus $B_{\xi}$ of $V$ with respect to any projection along $\xi$ as in Chirka [14, 2.7. In this particular situation we have

$$
B_{\xi}=\left\{z \in V: \frac{\partial f}{\partial \xi}(z)=0\right\},
$$

where $\frac{\partial f}{\partial \xi}$ denotes the directional derivative of $f$ in direction $\xi$.

4.3. Lemma. For each analytic surface $V$ in $\mathbb{C}^{3}$ which contains the origin, the following assertions hold:

(a) For each $\xi \in \mathbb{R}^{3} \backslash T_{0} V$ the set $B_{\xi}$ is either empty or an analytic variety of pure dimension 1 .

(b) There exists $q \in \mathbb{N}$ such that for each $\xi \in \mathbb{R}^{3} \backslash T_{0} V$ with $0 \in B_{\xi}$, each irreducible component $\left[W_{\xi}\right]_{0}$ of the germ $\left[B_{\xi}\right]_{0}$, and each basis $\left(b_{1}, b_{2}, b_{3}\right)$ of 
$\mathbb{C}^{3}$ with $b_{1} \in T_{0} W_{\xi}$, there are $a_{2, j}, a_{3, j} \in \mathbb{C}, j>q$, such that for a suitable neighborhood $U$ of the origin and suitable $\delta>0$

$$
W_{\xi} \cap U=\left\{t^{q} b_{1}+\sum_{j>q} a_{2, j} t^{j} b_{2}+\sum_{j>q} a_{3, j} t^{j} b_{3}:|t|<\delta\right\} .
$$

Proof. Note first that the case of a general surface $V$ can be reduced to the special case that $[V]_{0}$ is irreducible. To see this, choose $r>0$ such that $V$ is defined in $B(0, r)$ as the zero set of $f$ and such that the assumptions of Notation 4.1 are satisfied. Then we have for each $\xi \in \mathbb{R}^{3} \backslash T_{0} V$

$$
B_{\xi}(V)=\bigcup_{j=1}^{m} B_{\xi}\left(V_{j}\right) \cup \bigcup_{j, k=1, j \neq k}^{m} V_{j} \cap V_{k} .
$$

Hence it suffices to prove the lemma in the special case. In this situation we distinguish two cases:

Case 1: For each $\zeta \in S^{2}$ the function $\langle\nabla f, \zeta\rangle$ does not vanish identically. To prove (a) assume that for some $\xi \in S^{2}$ the assertion in (a) does not hold. Then $\operatorname{dim} B_{\xi}=2$ and it is no restriction to assume that $\xi=(1,0,0)$. Since $f$ is locally irreducible at zero, the assumption implies that $f$ is a factor of $\frac{\partial f}{\partial \xi}=\frac{\partial f}{\partial z_{1}}$. Then let

$$
W:=\left\{\left(z_{2}, z_{3}\right) \in B^{2}(0, r): f\left(0, z_{2}, z_{3}\right)=0\right\},
$$

and note that $0 \in V$ implies $0 \in W$, hence $\operatorname{dim} W \geq 1$. Next fix $\left(w_{2}, w_{3}\right) \in$ $W \backslash\{(0,0)\}$. If we assume that $h: z_{1} \mapsto\left(z_{1}, w_{2}, w_{3}\right)$ does not vanish identically, then there exists $k \in \mathbb{N}$ such that $h$ has a zero of order $k$ at the origin. Hence $\frac{\partial f}{\partial z_{1}}\left(\cdot, w_{2}, w_{3}\right)=\frac{\partial h}{\partial z_{1}}$ has a zero of order $k-1$ at the origin. Since $f$ is a factor of $\frac{\partial f}{\partial z_{1}}$, this leads to a contradiction. Hence $h$ vanishes identically. This implies that $f$ vanishes on $(\mathbb{C} \times W) \cap B^{3}(0, r)$. Since $f$ is irreducible it follows from this that $f=f(0, \cdot, \cdot)$ and hence $\frac{\partial f}{\partial z_{1}} \equiv 0$. Since this contradicts the hypothesis in the present case, we proved part (a) in this case.

For the proof of (b) in the present case note first that it is enough to consider $\xi \in \mathbb{R}^{3}$ with $|\xi|=1$. We show first: For each $\xi_{0} \in S^{2}$ (which may even be tangential) there are $M \in \mathbb{N}$ and $\delta>0$ such that for each $\xi \in S^{2}$ with $\left|\xi-\xi_{0}\right|<\delta$ the multiplicity $\mu_{0}\left(B_{\xi}\right)$ in the sense of Chirka [Ch], 11.1, does not exceed $M$.

To prove this, fix $\xi_{0} \in \mathbb{R}^{3}$. If $0 \in B_{\xi_{0}}$ we choose coordinates so that $\xi_{0}=(1,0,0)$ and so that each $\zeta=\left(\zeta_{1}, \zeta_{2}, \zeta_{3}\right) \in T_{0} B_{\xi_{0}} \backslash\{0\}$ satisfies $\zeta_{2} \neq 0$. Then for $\xi \in S^{2}$ we define

$$
F_{\xi}: B^{3}(0, r) \rightarrow \mathbb{C}^{3}, \quad F_{\xi}(z):=\left(f(z), \frac{\partial f}{\partial \xi}(z), z_{2}\right) .
$$

Note that $0 \in B_{\xi_{0}}$ implies $F_{\xi_{0}}(0)=0$, while the choice of the coordinates implies that the origin is an isolated zero of $F_{\xi_{0}}$. Hence there is a zero neighborhood $U \subset B^{3}(0, r)$ such that

$$
\left\{z \in \bar{U}: F_{\xi_{0}}(z)=0\right\}=\{0\} \text { and } \delta_{1}:=\min _{z \in \partial U}\left|F_{\xi_{0}}(z)\right|>0 .
$$

If $0 \notin B_{\xi_{0}}$, then define $F_{\xi}$ as above for some choice of coordinates. Then $F_{\xi}(0) \neq$ 0 and we can choose a zero neighborhood $U \subset B^{3}(0, r)$ and $\delta_{1}>0$ such that $\min _{z \in \bar{U}}\left|F_{\xi_{0}}(z)\right| \geq \delta_{1}$. Since $F_{\xi}$ depends continuously on $\xi$, there is $\delta>0$ such that

$$
\max _{z \in \partial U}\left|F_{\xi}(z)-F_{\xi_{0}}(z)\right|<\delta_{1} \leq \min _{z \in \partial U}\left|F_{\xi_{0}}(z)\right| \text { for } \xi \in S^{2} \text { with }\left|\xi-\xi_{0}\right|<\delta .
$$


Denote by $\mu_{0}\left(F_{\xi}\right)$ the multiplicity of the map $F_{\xi}$ as defined in Chirka [14, 10.2. Then Rouché's theorem [14, 10.3, Theorem 1, implies $\mu_{0}\left(F_{\xi}\right) \leq \mu_{0}\left(F_{\xi_{0}}\right)=: M$ if $\left|\xi-\xi_{0}\right|<\delta$. The multiplicity is defined as follows: Pick a neighborhood $U_{2}$ of 0 such that $F_{\xi}: U \rightarrow U_{2}$ is proper; then

$$
\mu_{0}\left(F_{\xi}\right)=\varlimsup_{w \rightarrow 0} \#\left(F_{\xi}^{-1}(w) \cap U\right) .
$$

In particular, for $z_{2}$ sufficiently small, there are at most $M$ points in $B_{\xi}$ whose second coordinate equals $z_{2}$. In other words, $\mu_{0}\left(B_{\xi}\right) \leq M$. Since $S^{2}$ is compact, this implies that

$$
m:=\max \left\{\mu_{0}\left(B_{\xi}\right): \xi \in S^{2}\right\}
$$

is a nonnegative integer. We define $q:=m$ !. To complete the proof of part (b), fix $\xi \in \mathbb{R}^{3} \backslash T_{0} V$ with $0 \in B_{\xi}$, let $\left[W_{\xi}\right]_{0}$ be an irreducible component of $\left[B_{\xi}\right]_{0}$, and pick a basis $\left(b_{1}, b_{2}, b_{3}\right)$ of $\mathbb{C}^{3}$ with $b_{1} \in T_{0} W_{\xi}$. By [14], 6.1, $W_{\xi}$ admits a Puiseux series expansion at the origin, i.e., there are $p \in \mathbb{N}$, a neighborhood $U$ of zero, and $a_{2, j}, a_{3, j} \in \mathbb{C}$ such that

$$
W_{\xi} \cap U=\left\{t^{p} b_{1}+\sum_{j=p+1}^{\infty} a_{2, j} t^{j} b_{2}+\sum_{j=p+1}^{\infty} a_{3, j} t^{j} b_{3}:|t|<\delta\right\} .
$$

This representation implies $p=\mu_{0}\left(W_{\xi}\right) \leq \mu_{0}\left(B_{\xi}\right)$. Hence $q$ is a multiple of $p$ and we may as well use $q$ instead of $p$.

Case 2: There exists $\zeta \in S^{2}$ such that $\langle\nabla f, \zeta\rangle \equiv 0$. It is no restriction to assume $\zeta=(0,0,1)$. Then $f$ is a function of the first two coordinates only. We start with the proof of (b). As $\xi \notin T_{0} V$, there is a neighborhood $U$ of 0 such that $V \cap U$ is given by a Weierstraß polynomial in $z_{1}$, which we assume to be $f$. The discriminant $\Delta$ of $f$ with respect to $z_{1}$ is an analytic function of $z_{2}$. Since $f$ is irreducible, it cannot share a common factor with $\frac{\partial f}{\partial z_{1}}$. Hence $\Delta \not \equiv 0$ and thus $\Delta$ admits a finite number $w_{1}, \ldots, w_{k}$ of zeros in a suitable neighborhood of the origin. Since $f$ is a Weierstraß polynomial, no function $f\left(\cdot, w_{j}\right)$ vanishes identically. Hence $B_{\xi}$ is the union of lines of the form $\left\{\left(z_{j, l}, w_{j}\right)\right\} \times \mathbb{C}$. This proves (b). Now claim (a) is immediate.

4.4. Definition. Let $V$ be an analytic surface in $\mathbb{C}^{3}$ which contains the origin, $\gamma$ a real simple curve, $d \geq 1$, and $A \neq \mathbb{C}^{3}$ a closed analytic set in $\mathbb{C}^{3}$. Then $\delta \geq d$ is called $A$-admissible for $\gamma$, if for each $\xi \in \mathbb{R}^{3} \backslash A$, each real simple curve $\tau$ which is equivalent to $\gamma$ modulo $d$ and satisfies $\operatorname{tr}(\tau) \subset B_{\xi}$ is already equivalent to $\gamma$ modulo $\delta$.

4.5. Lemma. Let $V$ be an analytic surface in $\mathbb{C}^{3}$ which contains the origin, $\gamma$ a real simple curve, and $A$ a closed analytic set in $\mathbb{C}^{3}$ which satisfies $T_{0} V \subset A \neq \mathbb{C}^{3}$. Then for each $d \geq 1$ there is $\left.\left.\Delta^{A}(\gamma, d) \in\right] d, \infty\right]$ such that

$$
\{\delta \geq d: \delta \text { is A-admissible for } \gamma\}=\left[d, \Delta^{A}(\gamma, d)[\text {. }\right.
$$

If $\Delta^{A}(\gamma, d)<\infty$, then $\Delta^{A}(\gamma, d)$ is not $A$-admissible for $\gamma$.

Proof. Obviously $d$ is $A$-admissible for $\gamma$. Hence it suffices to show that for each $\Delta \geq d$ which is $A$-admissible, there exists $\varepsilon>0$ such that each $\delta \in[\Delta, \Delta+\varepsilon[$ is $A$-admissible. To do this, fix such a number $\Delta, \xi \in \mathbb{R}^{3} \backslash A$, and a real simple curve $\tau$ which is equivalent to $\gamma$ modulo $d$ and satisfies $\operatorname{tr}(\tau) \subset B_{\xi}$. By Lemma 
4.3 there exists $q \in \mathbb{N}$, only depending on $V$ and not on $\tau$ such that for suitable $b_{j}(\xi) \in \mathbb{R}^{3}, j \geq q$, we have

$$
\tau(t)=\sum_{j=q}^{\infty} b_{j}(\xi) t^{j / q} .
$$

Since $\gamma$ and $\tau$ are equivalent modulo $\Delta$ we may assume by Lemmas 2.15(a) and 2.5 that both $\gamma$ and $\tau$ are in standard parametrization with respect to the same real basis of $\mathbb{R}^{3}$. Assume that in these coordinates, $\gamma(t)=\sum_{j=l}^{\infty} a_{j} t^{j / l}$ for some $l \in \mathbb{N}$. Then Lemma 2.15(b) implies that for some $\eta>0$ we have

$$
\left.\sum_{j=q}^{\Delta q} b_{j}(\xi) t^{j / q}=\sum_{j=l}^{\Delta l} a_{j} t^{j / l}, \quad t \in\right] 0, \eta[.
$$

Obviously, this implies that there exists $\varepsilon>0$, not depending on $\xi$ and $\tau$, such that (4.1) holds for $\Delta$ replaced by $\Delta+\varepsilon$. Hence $\gamma$ and $\tau$ are equivalent modulo $\delta$ for all $\delta \in[\Delta, \Delta+\varepsilon]$. This proves the first assertion. If $\Delta^{A}(\gamma, d)$ would be $A$-admissible, the previous argument shows that for some $\varepsilon>0$ all $\delta$ in $\left[d, \Delta^{A}(\gamma, d)+\varepsilon[\right.$ would be admissible, contrary to the definition of $\Delta^{A}(\gamma, d)$.

Remark. We do not know whether $\Delta^{A}(\gamma, d)$ actually depends on the choice of $A$ as long as $T_{0} V \cup T_{0}\left(T_{\gamma, d} V\right) \subset A$.

4.6. Definition. The number $\Delta^{A}(\gamma, d)$ from Lemma 4.5 is called the $A$-critical level of the pair $(\gamma, d)$.

4.7. Definition. For $V, \gamma, d$ as in Definition 4.4 and $\eta \in T_{\gamma, d} V \cap \mathbb{R}^{3}$, let $A:=$ $T_{\eta}\left(T_{\gamma, d} V\right) \cup T_{0} V$. We say that $\eta$ is a simple point of $T_{\gamma, d} V$ if there exist $\xi \in \mathbb{R}^{3} \backslash A$, a zero neighborhood $D$ in $\mathbb{C}^{3}$, and $\rho>0$ such that there is at most one real branch $T$ of $B_{\xi} \cap \mathbb{R}^{3}$ which is contained in $\Gamma(\gamma, d, \eta+D, \rho)$.

4.8. Lemma. Let $V, \gamma$, and $d \geq 1$ be as in Definition 4.4 and let $q$ denote the number from Lemma 4.3. Let $\eta \in T_{\gamma, d} V \cap \mathbb{R}^{3}$ be arbitrary if $d>1$, set $\eta=0$ if $d=1$, and set $\gamma_{\eta}: t \mapsto \gamma(t)+\eta t^{d}$ in both cases. Assume $\eta \in T_{\gamma, d} V \cap \mathbb{R}^{3}$ is not simple and let $A:=T_{\eta}\left(T_{\gamma, d} V\right) \cup T_{0} V$. Then $A=T_{0}\left(T_{\gamma_{\eta}, d} V\right) \cup T_{0} V$ and there exists $\nu \in \mathbb{N}, \nu>d q$, such that $\Delta^{A}\left(\gamma_{\eta}, d\right)=\frac{\nu}{q}$.

Proof. To show that $\Delta:=\Delta^{A}\left(\gamma_{\eta}, d\right)$ is finite, fix $\xi \in \mathbb{R}^{3} \backslash A$. Since $\eta$ is not a simple point of $T_{\gamma, d} V \cap \mathbb{R}^{3}$, there are at least two branches $S, T$ of $B_{\xi} \cap \mathbb{R}^{3} \backslash\{0\}$ which both have nonempty intersection with $\Gamma(\gamma, d, \eta+D, R)=\Gamma\left(\gamma_{\eta}, d, D, R\right)$ for each zero neighborhood $D$ in $\mathbb{C}^{3}$ and each $R>0$. Thus, $S$ and $T$ are equivalent to $\gamma_{\eta}$ modulo $d$. Since $S$ and $T$ are different, it follows from Lemmas 2.5 and 2.15 that we can choose $\delta>d$ so large that one of these branches is not equivalent to $\gamma_{\eta}$ modulo $\delta$. Hence $\delta$ is not $A$-admissible for $\gamma_{\eta}$ and we have $\Delta \leq \delta<\infty$.

Since $\Delta$ is not $A$-admissible for $\gamma_{\eta}$, there exist $\xi \in \mathbb{R}^{3} \backslash A$ and a real simple curve $\tau$ with $\operatorname{tr}(\tau) \subset B_{\xi}$ which is equivalent to $\gamma_{\eta}$ modulo $\Delta-\varepsilon$ for $0<\varepsilon<\Delta-1$ but which is not equivalent to $\gamma_{\eta}$ modulo $\Delta$. Since $\tau$ and $\gamma$ have the same tangent vector at the origin, it follows from Lemma 2.5 and Lemma 4.3 that we can choose standard parametrizations $\sum_{j=l}^{\infty} a_{j} t^{j / l}$ for $\gamma$ and $\sum_{j=q}^{\infty} b_{j} t^{j / q}$ for $\tau$ with respect to the same coordinates. Then it follows from Lemma 2.15 that for $0<\varepsilon<\Delta-d$ we 
have

$$
\sum_{j=l}^{(\Delta-\varepsilon) l} a_{j} t^{j / l}+\eta t^{d}=\sum_{j=q}^{(\Delta-\varepsilon) q} b_{j} t^{j / q}
$$

while

$$
\sum_{j=l}^{\Delta l} a_{j} t^{j / l}+\eta t^{d} \neq \sum_{j=q}^{\Delta q} b_{j} t^{j / q},
$$

where $\eta=0$ if $d=1$. It is easy to check that this implies $\Delta=\frac{\nu}{q}$ for some $\nu \in \mathbb{N}$. Since $d<\Delta$, we get $d q<\nu$. Since $T_{\gamma_{\eta}, d} V$ is the translate of $T_{\gamma, d}$ by $\eta$, we have $T_{\eta}\left(T_{\gamma, d} V\right)=T_{0}\left(T_{\gamma_{\eta}, d} V\right)$ and the proof is complete.

Remark. In Proposition 4.11 below, a lower estimate for $\Delta^{A}(\gamma, d)$ is derived under stronger hypotheses than those in Lemma 4.8.

4.9. Lemma. Let $V$ be an analytic surface in $\mathbb{C}^{3}$ which contains the origin and let $\gamma$ be a real simple curve in $\mathbb{R}^{3}$ with tangent vector $\xi$ at the origin. Then we have:

(a) Assume $d=1, \gamma(t)=\xi t$, and $\eta \in T_{\gamma, 1} V \cap \mathbb{R}^{3}$. Then $\eta$ is a simple point of $T_{\gamma, 1} V$ if and only if $\tau(\eta+\xi)-\xi$ is a simple point of $T_{\gamma, 1} V$ for each $\tau>0$.

(b) Assume $d>1$ and $\eta \in T_{\gamma, d} V \cap \mathbb{R}^{3}$. Then $\eta$ is a simple point of $T_{\gamma, d} V$ if and only if $\eta+\tau \xi$ is a simple point of $T_{\gamma, d} V$ for each $\tau \in \mathbb{R}$.

Proof. (a) By Theorem 2.7(b), we have $T_{\gamma, 1} V=T_{0} V-\xi$. Since $T_{0} V$ is a complex cone, it follows easily that

$$
\eta \in T_{\gamma, 1} V \cap \mathbb{R}^{3} \text { if and only if } \tau(\eta+\xi)-\xi \in T_{\gamma, 1} V \cap \mathbb{R}^{3} \text { for each } \tau \in \mathbb{R}
$$

and that

$$
T_{\eta}\left(T_{\gamma, 1} V\right)=T_{\eta}\left(T_{0} V-\xi\right)=T_{\eta+\xi}\left(T_{0} V\right)=T_{\tau(\eta+\xi)}\left(T_{0} V\right)=T_{\tau(\eta+\xi)-\xi}\left(T_{\gamma, 1} V\right) .
$$

Next note that for each zero neighborhood $D$ in $\mathbb{C}^{3}, R>0$, and $\tau>0$, we have

$$
\begin{aligned}
\Gamma(\gamma, 1, \tau & \left.(\eta+\xi)-\xi, \tau D, \frac{R}{\tau}\right)=\bigcup_{0<s<R / \tau} s \xi+s(\tau(\eta+\xi)-\xi+\tau D) \\
= & \bigcup_{0<s<R / \tau} s \tau(\eta+\xi)+s \tau D=\bigcup_{0<t<R} \xi t+t(\eta+D)=\Gamma(\gamma, 1, \eta+D, R) .
\end{aligned}
$$

From these facts it is now obvious that (a) holds.

(b) Note first that by Theorem 2.7(c), $\eta \in T_{\gamma, d} V$ is equivalent to $\eta+\tau \xi \in T_{\gamma, d} V$ for each $\tau \in \mathbb{C}$ and that therefore

$$
T_{\eta}\left(T_{\gamma, d} V\right)=T_{\eta+\tau \xi}\left(T_{\gamma, d} V\right) \text { for each } \tau \in \mathbb{C} .
$$

As in part (a) the claim follows if we show that for each $\tau \in \mathbb{R}$, each absolutely convex zero neighborhood $D$ in $\mathbb{C}^{3}$, and each $R>0$, there are zero neighborhoods $D_{1}, D_{2}$ in $\mathbb{C}^{3}$ and $R_{1}, R_{2}<R$ such that

$$
\begin{aligned}
\Gamma\left(\gamma, d, \eta+D, R_{1} / 2\right) & \subset \Gamma\left(\gamma, d, \eta+\tau \xi+D_{1}, R_{1}\right) \text { and } \\
\Gamma\left(\gamma, d, \eta+\tau \xi+D, R_{2} / 2\right) & \subset \Gamma\left(\gamma, d, \eta+D_{2}, R_{2}\right) .
\end{aligned}
$$

To prove this, fix $\tau, D$, and $R$ and assume that $\gamma(t)=\xi t+\sigma(t)$, where $\sigma(t)=$ $\sum_{j=q+1}^{\infty} a_{j} t^{j / q}$ for $0<t \leq \alpha$. Then there is $C>0$ such that

$$
\left|\sigma^{\prime}(t)\right| \leq C t^{1 / q} \text { for } 0<t \leq \alpha / 2 .
$$


If we let $s:=s(t):=t+\tau t^{d}$, it follows from $d>1$ that there is $t_{0}>0$ such that $0<s(t)<R$ if $0<t<t_{0}$. By the mean value theorem we get

$$
|\sigma(t)-\sigma(s)| \leq C \sup _{0<\vartheta<1}|t+\vartheta(s-t)|^{1 / q} \cdot|t-s| \leq C\left(t+\tau t^{d}\right)^{1 / q} \tau t^{d}
$$

and hence

$$
\sigma(t)-\sigma(s(t)) \in t^{d} D \text { whenever } 0<t<R_{1} .
$$

Next note

$$
\left|\left(1+\tau t^{d-1}\right)^{d}-1\right| \leq\left(1+\tau t^{d-1}\right)^{d-1} \tau^{d} t^{d(d-1)} \leq 2 \tau^{d} t^{d(d-1)} .
$$

This implies

$$
s^{d}(\eta+D)=t^{d}(\eta+D)+t^{d}\left[\left(1+\tau t^{d-1}\right)^{d}-1\right](\eta+D) \subset t^{d}(\eta+2 D)
$$

whenever $0<t<R_{1}$, provided that $R_{1}$ is small enough. Altogether we proved that

$$
\begin{aligned}
\gamma(s)+s^{d}(\eta+D) & =s \xi+\sigma(s)+s^{d}(\eta+D)=t \xi+t^{d}(\eta+\tau \xi+D) \\
& =t \xi+\sigma(t)+t^{d}(\eta+\tau \xi+D)+(\sigma(s)-\sigma(t))+s^{d}(\eta+D) \\
& \subset \gamma(t)+t^{d}(\eta+\tau \xi+3 D) .
\end{aligned}
$$

From this we get the first assertion in (4.2). The second one follows by the same arguments.

Remark. Under the hypothesis of Lemma 4.9, for $\eta \in T_{0} V \cap S^{2}$ and $\xi, \zeta \in S^{2}, \eta-\xi$ is a simple point of $T_{\gamma_{\xi}, 1} V$ if and only if $\eta-\zeta$ is a simple point of $T_{\gamma_{\zeta}, 1} V$, where $\gamma_{\xi}: t \mapsto t \xi, \gamma_{\zeta}: t \mapsto t \zeta$. This is true since both conditions mean that there are $\lambda \in \mathbb{R}^{3} \backslash\left(T_{\eta}\left(T_{0} V\right) \cup T_{0} V\right)$, a zero neighborhood $D$ in $\mathbb{C}^{3}$, and $\rho>0$ such that there is at most one real branch $T$ of $B_{\xi} \cap \mathbb{R}^{3}$ which is contained in $\Gamma\left(\gamma_{\eta}, 1, D, \rho\right)$.

4.10. Lemma. Let $V$ be an analytic surface in $\mathbb{C}^{3}$ which contains the origin, and let $\gamma$ be a real simple curve with tangent vector $\xi$ in the origin which is in standard parametrization $\gamma(t)=\sum_{j=q}^{\infty} b_{j} t^{j / q}$, where $q$ is the number from Lemma 4.3. Let

$$
\mathcal{M}_{\gamma, d}:=\left\{\eta \in T_{\gamma, d} V \cap \mathbb{R}^{3}: \eta \text { is not simple }\right\} .
$$

(a) If $d=1$ and $\gamma(t)=\xi$ t, then the set

$$
M:=\left\{\zeta \in T_{0} V \cap S^{2}: \zeta-\xi \in \mathcal{M}_{\gamma, 1}\right\}
$$

is finite and $\mathcal{M}_{\gamma, 1} \backslash\{-\xi\}=\bigcup_{\zeta \in M}\{\tau \zeta-\xi: \tau>0\}$.

(b) If $d=\frac{\nu}{q}$ for some $\nu>q$, then the set

$$
M:=\left\{\zeta \in \mathcal{M}_{\gamma, d}:\langle\zeta, \xi\rangle=0\right\}
$$

is finite and $\mathcal{M}_{\gamma, d}=\bigcup_{\zeta \in M}\{\zeta+\tau \xi: \tau \in \mathbb{R}\}$.

Proof. (a) To argue by contradiction, assume that $M$ is not finite. Then choose a sequence $\left(\zeta_{n}\right)_{n \in \mathbb{N}}$ in $M$ and let

$$
A:=\bigcup_{n \in \mathbb{N}} T_{\zeta_{n}-\xi}\left(T_{\gamma, 1} V\right) \cup T_{0} V
$$

Since $A$ is homogeneous and pluripolar in $\mathbb{C}^{3}$ (for the definition see Klimek [19, Chapter 2.9), but $\mathbb{R}^{3}$ is not, there exists $\xi_{0} \in \mathbb{R}^{3} \backslash A,\left|\xi_{0}\right|=1$. By Lemma 4.3 , there exist real simple curves $\tau_{1}, \ldots, \tau_{m}$ and a zero neighborhood $U$ in $\mathbb{R}^{3}$ such that

$$
B_{\xi_{0}} \cap U=\bigcup_{j=1}^{m} \operatorname{tr}\left(\tau_{j}\right) \cap U .
$$


For $1 \leq j \leq m$ let $\xi_{j}$ be the tangent vector of $\tau_{j}$ at the origin, fix $n \in \mathbb{N}$, let $\zeta:=\zeta_{n}$, and define $\gamma_{\zeta}: t \mapsto \zeta t$. Since $\zeta-\xi$ is not simple and since $\xi_{0} \in \mathbb{R}^{3} \backslash A \subset$ $\mathbb{R}^{3} \backslash\left(T_{\zeta-\xi}\left(T_{\gamma, 1} V\right) \cup T_{0} V\right)$, there are at least two real simple curves $\tau$ which satisfy

$$
\operatorname{tr}(\tau) \subset B_{\xi_{0}} \cap \mathbb{R}^{3} \text { and } \operatorname{tr}(\tau) \cap \Gamma(\gamma, 1, \zeta-\xi+D, R) \neq \emptyset
$$

for each zero neighborhood $D$ and each $R>0$. Since

$$
\Gamma(\gamma, 1, \zeta-\xi+D, R)=\bigcup_{0<t<R} t \xi+t(\zeta-\xi+D)=\Gamma\left(\gamma_{\zeta}, 1, D, R\right),
$$

there exists $1 \leq i \leq m$ such that $\gamma_{\zeta}$ is equivalent to $\tau_{i}$ modulo 1. By Lemma 2.15(a) this implies that $\tau_{i}$ and $\gamma_{\zeta}$ have the same tangent vector $\zeta$ at the origin, i.e., $\zeta \in\left\{\xi_{1}, \ldots, \xi_{m}\right\}$. Consequently, the set $M$ has at most $m$ elements, in contradiction to the assumption that $M$ is infinite. This proves the first part of (a).The second one follows immediately from the first one by Lemma 4.9(a) and Theorem 2.7(b).

(b) It is no restriction to assume $\xi=(1,0,0)$ and that $\gamma$ is in standard parametrization with respect to the canonical basis of $\mathbb{R}^{3}$. If we assume that $M$ is not finite, we can find an infinite set $\left\{\zeta_{j}: j \in \mathbb{N}\right\}$ in $M$. Arguing as in part (a) we find $\xi_{0} \in \mathbb{R}^{3}$ such that (4.3) holds and such that for each $\zeta$ in $\left\{\zeta_{j}: j \in \mathbb{N}\right\}$ there is $\tau_{i}$ as in part (a) satisfying

$$
\operatorname{tr}\left(\tau_{i}\right) \cap \Gamma(\gamma, d, \zeta+D, R) \neq \emptyset
$$

for each zero neighborhood $D$ in $\mathbb{C}^{3}$ and each $R>0$. Now define $\gamma_{\zeta}: t \mapsto \gamma(t)+\zeta t^{d}$. Since $\zeta \in M, \zeta_{1}=0$ and $\gamma_{\zeta}$ is in standard parametrization with respect to the canonical basis. Since

$$
\Gamma(\gamma, d, \zeta+D, R)=\bigcup_{0<t<R}\left(t, \gamma_{2}(t), \gamma_{3}(t)\right)+t^{d}\left(\left(0, \zeta_{2}, \zeta_{3}\right)+D\right)=\Gamma\left(\gamma_{\zeta}, d, D, R\right),
$$

$\tau_{i}$ is equivalent to $\gamma_{\zeta}$ by (4.4). From this and Lemmas 2.15, 2.5, and 4.3 we get that there is a standard parametrization for $\tau_{i}$ with respect to the same coordinates as for $\gamma_{\zeta}$ which is of the form $\tau_{i}(t)=\sum_{j=q}^{\infty} a_{i, j}{ }^{j / q}$. Hence the hypotheses on $\gamma$ and Lemma 2.15(b) imply $a_{i, \nu}=b_{\nu}+\zeta$. Since there are at most $k$ different choices for $a_{i, \nu}$, it follows that $\left\{\zeta_{j}: j \in \mathbb{N}\right\}$ is finite in contradiction to the assumption. By this contradiction, the first part of (a) is proved. The second one follows from Lemma 4.9(b).

4.11. Proposition. For some $r>0$ let $f: B^{3}(0, r) \rightarrow \mathbb{C}$ be a holomorphic function which is real over real points and vanishes at zero. Let $\gamma$ be a real simple curve, let $d \geq 1$ be given, and assume that in the notation of Example 7.6 we have $d_{\nu} \leq$ $d<d_{\nu+1}$ for $1 \leq \nu<p$. Let $V:=V(f)$ and $A:=T_{0}\left(T_{\gamma, d} V\right) \cup T_{0} V$. Assume furthermore that $\left(F_{\omega_{0}\left(d_{\nu}\right)}(1, \cdot)\right)_{0}$ is square-free and that 0 is not a simple point of $T_{\gamma, d} V$. Then $\Delta^{A}(\gamma, d) \geq d_{\nu+1}$.

Proof. To argue by contradiction, we assume $\Delta:=\Delta^{A}(\gamma, d)<d_{\nu+1}$. Then Lemma 4.5 implies $d_{\nu} \leq d<\Delta<d_{\nu+1}$. Since 0 is not a simple point of $T_{\gamma, d} V$, there exist $\xi \in \mathbb{R}^{3} \backslash A$ and a real simple curve $\sigma$ satisfying $\operatorname{tr}(\sigma) \subset B_{\xi}$ such that $\sigma$ is equivalent to $\gamma$ modulo $d$ but not modulo $\Delta$. Consequently, $\gamma$ and $\sigma$ have the same tangent vector at the origin. We choose a basis $\left(e_{1}, e_{2}, e_{3}\right)$ so that this tangent vector equals $e_{1}=(1,0,0)$. Since $e_{1} \in T_{0} V$, we may assume $\xi=e_{3}=(0,0,1)$. By Lemma 2.5 we may assume that $\gamma$ and $\sigma$ are in standard parametrization with respect to this 
basis, say $\gamma(t)=\left(t, \gamma_{2}(t), \gamma_{3}(t)\right)$ and $\sigma(t)=\left(t, \sigma_{2}(t), \sigma_{3}(t)\right)$. If $\gamma(t)=\sum_{j=q}^{\infty} a_{j} t^{j / q}$ and $\sigma(t)=\sum_{j=q}^{\infty} b_{j} t^{j / q}$, then the present hypotheses imply by Lemma 2.15

$$
\sum_{j=q}^{(\Delta-\varepsilon) q} a_{j} t^{j / q}=\sum_{j=q}^{(\Delta-\varepsilon) q} b_{j} t^{j / q} \text { for } 0<t<t_{0} \text { and } 0<\varepsilon<\Delta-1
$$

and

$$
\liminf _{t \downarrow 0} t^{-\Delta}|\gamma(t)-\sigma(t)|>0 .
$$

Next consider the expansion

$$
f(\gamma(t)+z)=\sum_{\alpha \in \mathbb{N}_{0}^{3}, j \geq q} a_{j, \alpha} z^{\alpha} t^{j / q} .
$$

Note that for some $\rho>0$ the series converges absolutely and uniformly for $|z| \leq \rho$ and $0 \leq t \leq \rho$. Using the notation that was introduced in Definition 2.10 and Remark 2.11 denote by $P$ the localization of $F_{\omega_{0}\left(d_{\nu}\right)}(1, \cdot)$ at zero, i.e.,

$$
P:=\left(F_{\omega_{0}\left(d_{\nu}\right)}(1, \cdot)\right)_{0} .
$$

Then $P$ is homogeneous of degree $m$ and does not depend on the first variable. The latter fact follows from Theorem 2.7(c) if $\nu>1$ and from the fact that $e_{1}$ is the tangent vector of $\gamma$ at the origin if $\nu=1$. By hypothesis, $P$ is square-free and by Proposition 2.12(a) we have

$$
F_{\omega_{0}(\delta)}(1, \cdot)=\left(F_{\omega_{0}\left(d_{\nu}\right)}(1, \cdot)\right)_{0}=P \text { for } d_{\nu}<\delta<d_{\nu+1} .
$$

From (4.8), (2.3), and the homogeneity of $P$ we get $T_{0}\left(T_{\gamma, d} V\right)=V(P)$. Consequently $e_{3}=\xi \notin A$ is noncharacteristic for $P$. Therefore there are pairwise different complex numbers $\beta_{1}, \ldots, \beta_{m}$ and $B \in \mathbb{C} \backslash\{0\}$ such that

$$
P\left(w_{2}, w_{3}\right)=B \prod_{j=1}^{m}\left(w_{3}-\beta_{j} w_{2}\right) .
$$

It is no restriction to assume $B=1$.

Next choose $D>0$ and $\rho>0$ such that

$$
\sum_{\alpha \in \mathbb{N}_{0}^{3}, j \geq q}\left|a_{j, \alpha} z^{\alpha} t^{j / q}\right| \leq D \text { for }|z| \leq \rho, 0 \leq t \leq \rho .
$$

Then regroup the expansion (4.7) according to the procedure described in Definition 2.10 , i.e., for $d_{\nu}<\delta<d_{\nu+1}$ let

$$
\omega_{0}:=\omega_{0}(\delta):=\min \left\{\delta|\alpha|+\frac{j}{q}: a_{j, \alpha} \neq 0\right\}
$$

and choose an increasing sequence $\left(\omega_{k}\right)_{k \in \mathbb{N}}=\left(\omega_{k}(\delta)\right)_{k \in \mathbb{N}}$ such that for

$$
F_{\omega_{k}}(t, w):=\sum_{\delta|\alpha|+\frac{j}{q}=\omega_{k}} a_{j, \alpha} w^{\alpha} t^{j / q}
$$

we have

$$
f\left(\gamma(t)+t^{\delta} w\right)=\sum_{k \in \mathbb{N}_{0}} F_{\omega_{k}}\left(t, t^{\delta} w\right)=\sum_{k \in \mathbb{N}_{0}} t^{\omega_{k}} F_{\omega_{k}}(1, w)
$$


From this and (4.8) we get for $d_{\nu}<\delta<d_{\nu+1}$

$$
t^{-\omega_{0}(\delta)} f\left(\gamma(t)+t^{\delta} w\right)=P(w)+\sum_{k \in \mathbb{N}} t^{\omega_{k}(\delta)-\omega_{0}(\delta)} F_{\omega_{k}(\delta)}(1, w) .
$$

Now we claim that

$$
\lim _{t \downarrow 0} \frac{1}{t^{\Delta}}\left(\sigma_{2}(t)-\gamma_{2}(t)\right)=b \neq 0 .
$$

To prove this, note that $\sigma_{2}-\gamma_{2}$ is given by a Puiseux series. Hence it follows from (4.5) that the limit exists. If we assume the limit is zero, then there exists $\varepsilon>0$ such that

$$
\frac{1}{t^{\Delta}}\left|\sigma_{2}(t)-\gamma_{2}(t)\right|=o\left(t^{\varepsilon}\right) .
$$

Then (4.6) implies by the same arguments

$$
\lim _{t \downarrow 0} \frac{1}{t^{\Delta}}\left(\sigma_{3}(t)-\gamma_{3}(t)\right)=d \neq 0 .
$$

Now let $\kappa(t):=t^{-\Delta}(\sigma(t)-\gamma(t))$ and note that $\operatorname{tr}(\sigma) \subset B_{\xi}$ and (4.10) imply for $0<t \leq \rho$ and $\delta=\Delta$

$$
\begin{aligned}
0 & =t^{-\omega_{0}} f(\sigma(t))=t^{-\omega_{0}} f\left(\gamma(t)+t^{\Delta} \kappa(t)\right) \\
& =P(\kappa(t))+t^{\frac{1}{q}} \sum_{k \in \mathbb{N}} t^{\omega_{k}-\omega_{0}-1 / q} F_{\omega_{k}}(1, \kappa(t)) .
\end{aligned}
$$

From (4.12) and (4.13) we get that $\kappa(t)$ is bounded on $] 0, \rho / 2[$ and that

$$
P(\kappa(t))=\prod_{j=1}^{m}\left(\kappa_{3}(t)-\beta_{j} \kappa_{2}(t)\right) \rightarrow d^{m} \text { as } t \rightarrow 0 .
$$

Hence in the limit for $z \rightarrow 0$ we get from (4.14) the contradiction $d^{m}=0$. Therefore we must have (4.11).

Next we claim that the following assertion holds:

There exist $r_{0}>0, M_{0} \geq 1$, and $0<\kappa_{0}<1$ such that for each $d_{\nu}<\delta<d_{\nu+1}, M \geq M_{0}$, and $0<\mu \leq \kappa_{0}$ there exist $\rho_{0}>0$ and $C \geq 1$ such that for each $0<t \leq \rho_{0}$ and each $w_{2} \in \mathbb{C}$ satisfying $C t^{d_{\nu+1}-\delta} \leq\left|w_{2}\right| \leq \frac{r_{0}}{M+1}$ the function $w_{3} \mapsto f\left(\gamma(t)+t^{\delta}\left(0, w_{2}, w_{3}\right)\right)$ has exactly $m$ different zeros of multiplicity one in the disk $\left|w_{3}\right| \leq M\left|w_{2}\right|$, each lying in one of the disks $B^{1}\left(\beta_{j} w_{2}, \mu\left|w_{2}\right|\right), 1 \leq j \leq m$.

To prove this claim using the theorem of Rouché, let $r_{0}:=\min (1, \rho)$, where $\rho$ is chosen as in (4.9). Furthermore let

$$
\begin{gathered}
M_{0}:=1+\max \left\{\left|\beta_{j}\right|: 1 \leq j \leq m\right\}, \\
\kappa:=\frac{1}{2} \min \left\{\left|\beta_{j}-\beta_{k}\right|: 1 \leq j<k \leq m\right\}, \kappa_{0}:=\min \left(\frac{3}{4}, \kappa\right) .
\end{gathered}
$$

It is easy to check that for $w_{2} \in \mathbb{C} \backslash\{0\}, M \geq M_{0}$, and $0<\mu \leq \kappa_{0}$ the following estimates hold:

$$
\begin{gathered}
\min \left\{\left|P\left(w_{2}, w_{3}\right)\right|:\left|w_{3}\right|=M\left|w_{2}\right|\right\} \geq\left|w_{2}\right|^{m}, \\
\min \left\{\left|P\left(w_{2}, w_{3}\right)\right|:\left|w_{3}-\beta_{j} w_{2}\right|=\mu\left|w_{2}\right|\right\} \geq\left(\mu\left|w_{2}\right|\right)^{m}, 1 \leq j \leq m .
\end{gathered}
$$


Now fix $\delta$ satisfying $d_{\nu}<\delta<d_{\nu+1}$ and assume that $w_{2} \in \mathbb{C}$ satisfies

$$
C t^{d_{\nu+1}-\delta} \leq\left|w_{2}\right| \leq \frac{r_{0}}{M+1}
$$

where the number $C$ will be determined later. To estimate the series in the expansion (4.10), let $k_{0}:=\min \left\{k \in \mathbb{N}: k>q m\left(d_{\nu+1}-\delta\right)\right\}$. Then we have for $0 \leq t \leq \rho$ and $|w| \leq \rho$ by the hypotheses of (4.15) and by (4.9)

$$
\left|\sum_{k \geq k_{0}} t^{\omega_{k}-\omega_{0}} F_{\omega_{k}}(1, w)\right| \leq D t^{k_{0} / q}=o\left(t^{m\left(d_{\nu+1}-\delta\right)}\right)=o\left(|w|^{m}\right) .
$$

This implies the existence of $0<\rho_{1} \leq \rho$ such that for $0<t \leq \rho_{1},\left|\left(w_{1}, w_{3}\right)\right| \leq M\left|w_{2}\right|$ and $\left|w_{2}\right| \leq \frac{r_{0}}{M+1}$ we get

$$
\left|\sum_{k \geq k_{0}} t^{\omega_{k}-\omega_{0}} F_{\omega_{k}}(1, w)\right| \leq \frac{1}{3}\left(\mu\left|w_{2}\right|\right)^{m} .
$$

To estimate $t^{k / q} F_{\omega_{k}}(1, w)$ for $1 \leq k<k_{0}$, denote by $m_{k}$ the order of zero of $F_{\omega_{k}}(1, \cdot)$ at the origin. Then there exists $D_{k}>0$ such that $\left|F_{\omega_{k}}(1, w)\right| \leq D_{k}|w|^{m_{k}}$ for $|w| \leq 1$. If $m_{k} \geq m$, then choose $0<\rho_{2}(k) \leq \rho$ such that for $0<t \leq \rho_{2}(k)$, $\left|\left(w_{1}, w_{3}\right)\right| \leq M\left|w_{2}\right|$ and $\left|w_{2}\right| \leq 1$ we have

$$
t^{k / q}\left|F_{\omega_{k}}(1, w)\right| \leq t^{1 / q} D_{k}|w|^{m_{k}} \leq t^{1 / q} D_{k}(M+1)^{m}\left|w_{2}\right|^{m} \leq \frac{1}{3 k_{0}}\left(\mu\left|w_{2}\right|\right)^{m} .
$$

To get a similar estimate when $m_{k}<m$, note that in this case, in $F_{\omega_{k}}(t, w)$ there is a term $a_{j, \alpha} z^{\alpha} t^{j / q}$ satisfying $a_{j, \alpha} \neq 0$ and $|\alpha|=m_{k}$. This implies $\omega_{k}(\delta)=$ $\delta m_{k}+j / q$. Now note that by $(2.6)$ we have

$$
\omega_{0}(\delta)=\delta l_{n}+\frac{j_{n}}{q}=\delta m+\frac{j_{n}}{q},
$$

because $m$ is the degree of $P=\left(F_{\omega_{0}(\delta)}(1, w)\right)_{0}$. Since $m>m_{k}$ and $\omega_{0}(\delta)<\omega_{k}(\delta)$, we must have $j>j_{n}$. Next note that the Newton polygon admits a supporting line of slope $-\frac{1}{\delta}$ touching at $\left(\frac{j}{q}, m_{k}\right)$. Since $d_{\nu}<\delta<d_{\nu+1}$, this implies that the Newton polygon also admits a supporting line of slope $-1 / d_{\nu+1}$ through $\left(\frac{j}{q}, m_{k}\right)$. This gives

$$
\left(\frac{j}{q}-\frac{j_{n}}{q}\right)^{-1}\left(m_{k}-m\right) \geq-\frac{1}{d_{\nu+1}}, \text { hence } \frac{j-j_{n}}{q\left(m-m_{k}\right)} \leq d_{\nu+1} .
$$

From this we get

$$
\frac{\omega_{k}(\delta)-\omega_{0}(\delta)}{m-m_{k}}=\left(m-m_{k}\right)^{-1}\left(\delta m_{k}+\frac{j}{q}-\delta m-\frac{j_{n}}{q}\right)=-\delta+\frac{j-j_{n}}{q\left(m-m_{k}\right)} \leq d_{\nu+1}-\delta=: a .
$$

By (4.18) this implies $0<t<\left(\frac{\left|w_{2}\right|}{C}\right)^{1 / a}$. For $\left|\left(w_{1}, w_{3}\right)\right| \leq M\left|w_{2}\right|$ and $\left|w_{2}\right| \leq \frac{r_{0}}{M+1}$, we now get

$$
\begin{aligned}
& t^{k / q}\left|F_{\omega_{k}}(1, w)\right|=t^{\omega_{k}-\omega_{0}}\left|F_{\omega_{k}}(1, w)\right| \leq t^{\omega_{k}-\omega_{0}} D_{k}|w|^{m_{k}} \\
& \quad \leq D_{k} C^{\left(\omega_{0}-\omega_{k}\right) / a}\left|w_{2}\right|^{\left(\omega_{k}-\omega_{0}\right) / a}|w|^{m_{k}} \leq D_{k}(M+1)^{m_{k}} C^{\left(\omega_{0}-\omega_{k}\right) / a}\left|w_{2}\right|^{m} .
\end{aligned}
$$

Since $\left(\omega_{0}-\omega_{k}\right) / a<0$, we can choose $C>0$ so large that

$$
t^{k / q}\left|F_{\omega_{k}}(1, w)\right| \leq \frac{1}{3 k_{0}}\left(\mu\left|w_{2}\right|\right)^{m}
$$

also in this case. 
From (4.19), (4.20), and (4.21) it follows that there is $C \geq 1$ such that for $\rho_{0}:=\min \left\{\rho_{1}, \rho_{2}(k): 1 \leq k<k_{0}, m_{k}>m\right\}$ we get for $0<t \leq \rho_{0},\left|w_{3}\right| \leq M\left|w_{2}\right|$, and $C t^{d_{\nu+1}-\delta} \leq\left|w_{2}\right| \leq \frac{r_{0}}{M+1}$ from (4.10) that

$$
t^{-\omega_{0}(\delta)}\left|f\left(\gamma(t)+t^{\delta}\left(0, w_{2}, w_{3}\right)\right)-P\left(w_{2}, w_{3}\right)\right|<\left(\mu\left|w_{2}\right|\right)^{m} .
$$

Since $0<\mu \leq \kappa_{0} \leq 1$, our claim now follows from (4.22), (4.16), and (4.17) by the theorem of Rouché.

To complete the proof, let $r_{0}, M_{0}$ and $\kappa_{0}$ be the constants from (4.15), fix $d_{\nu}<$ $\delta<\Delta$ and let

$$
\lambda(t):=t^{\Delta-\delta} \kappa(t)=\frac{1}{t^{\delta}}(\sigma(t)-\gamma(t)) .
$$

Since (4.11) and (4.6) imply

$$
\lim _{t \downarrow 0} \kappa_{2}(t)=b, \lim _{t \downarrow 0} \kappa_{3}(t)=c,
$$

where $b \neq 0$, we can choose $M \geq M_{0}$ such that

$$
\left|\lambda_{3}(t)\right| \leq M\left|\lambda_{2}(t)\right|
$$

For this choice of $M$ and $\mu:=\kappa_{0}$, choose $\rho_{0}>0$ and $C>1$ according to (4.15). Then (4.23) and $\Delta-\delta>0$ imply the existence of $0<\rho_{1} \leq \rho_{0}$ such that for $0<t \leq \rho_{1}$ we have

$$
\left|\lambda_{2}(t)\right| \leq 2|b| t^{\Delta-\delta} \leq \frac{r_{0}}{M+1} .
$$

Moreover, since $\Delta-d_{\nu+1}<0$, there exists $0<\rho_{2} \leq \rho_{0}$ such that

$$
\left|\lambda_{2}(t)\right| \geq \frac{1}{2}|b| t^{\Delta-\delta}=\frac{1}{2 C}|b| t^{\Delta-d_{\nu+1}} C t^{d_{\nu+1}-\delta} \geq C t^{d_{\nu+1}-\delta} .
$$

Since $t^{-\omega_{0}(\delta)} f\left(\gamma(t)+t^{\delta} \lambda(t)\right)=t^{-\omega_{0}(\delta)} f(\sigma(t))=0$, we now get from (4.15) that for $0<t \leq \min \left(\rho_{1}, \rho_{2}\right)$ there exists $1 \leq j \leq m$, such that $\lambda_{3}(t)$ is a simple zero of

$$
w_{3} \mapsto f\left(\gamma(t)+t^{\delta}\left(0, \lambda_{2}(t), w_{3}\right)\right) .
$$

Since $\sigma(t)=\gamma(t)+t^{\delta} \lambda(t)$ is a branch point of $V=V(f)$ with respect to the projection along $\xi=e_{3}$, this is a contradiction. By this contradiction our assumption at the beginning is false. Hence we must have $\Delta \geq d_{\nu+1}$.

\section{SUFFICIENCY}

In this section we will prove the main theorem of this paper, which states that the necessary conditions that were derived in section 3 are also sufficient. In fact we will prove more, namely, that only a finite set of conditions have to be satisfied. To formulate this result, we first construct a specific set of real simple curves.

5.1. Construction of a particular finite set $\mathcal{C}$. Let $V$ be an analytic surface in $\mathbb{C}^{3}$ which contains the origin and denote by $q$ the number from Lemma 4.3. For $\eta \in S^{2}$ define $\sigma_{\eta}: t \mapsto t \eta, t>0$. To begin the construction, we first consider the set of real singular directions in $T_{0} V$ for which there are several real branch curves of $V$ nearby with respect to most real directions. More precisely, for $\xi \in S^{2}$ this set is defined as

$$
M_{1}:=\left\{\eta \in\left(T_{0} V\right)_{\operatorname{sing}} \cap S^{2}: \eta-\xi \text { is not a simple point of } T_{\sigma_{\xi}, 1} V\right\}
$$


and does not depend on $\xi$ by the Remark following Lemma 4.9. If $M_{1}=\emptyset$, then $\mathcal{C}:=\emptyset$. Otherwise let

$$
\mathcal{C}_{0}:=\left\{\left(\sigma_{\eta}, 1\right): \eta \in M_{1}\right\}
$$

We are going to recursively define sets $\mathcal{C}_{j}, j \in \mathbb{N}$, satisfying the following condition:

$\mathcal{C}_{j}$ is finite and for each $(\gamma, d) \in \mathcal{C}_{j}$ there is $\nu \in \mathbb{N}, \nu \geq q+j$, such that $d=\frac{\nu}{q}$ and $\gamma$ has a standard parametrization of the form $\gamma(t)=\sum_{i=q}^{\nu-1} a_{i} t^{i / q}$.

To start the recursive definition, let $A(\eta):=T_{\eta}\left(T_{0} V\right) \cup T_{0} V$ and denote by $\Delta^{A(\eta)}\left(\sigma_{\eta}, 1\right)$ the $A(\eta)$-critical level of $\sigma_{\eta}$, according to Definition 4.6. For $\eta \in M_{1}$, it follows from Lemma 4.8 that $q \Delta^{A(\eta)}\left(\sigma_{\eta}, 1\right)=\nu(\eta) \in \mathbb{N}, \nu(\eta) \geq q+1$. Since $M_{1}$ is finite by Lemma 4.10 , the set $\mathcal{C}_{1}$ defined as

$$
\mathcal{C}_{1}:=\left\{\left(\sigma_{\eta}, \Delta^{A(\eta)}\left(\sigma_{\eta}, 1\right)\right): \eta \in M_{1}\right\}
$$

satisfies the conditions in (5.1) for $j=1$.

Assume now that for some $j \in \mathbb{N}, j \geq 2$, the set $\mathcal{C}_{j-1}$ is defined in such a way that (5.1) holds for $j-1$ and that $\mathcal{C}_{j-1}$ is finite. To define $\mathcal{C}_{j}$, let

$\mathcal{C}_{j-1, c}:=\left\{(\gamma, d) \in \mathcal{C}_{j-1}: \quad\right.$ there exists $\quad \zeta \in\left(T_{\gamma, d} V\right)_{\operatorname{sing}} \cap \mathbb{R}^{3}, \quad \zeta$ is not simple $\}$. If $\mathcal{C}_{j-1, c}=\emptyset$, then let $\mathcal{C}_{j}:=\emptyset$. Otherwise fix $(\gamma, d) \in \mathcal{C}_{j-1, c}$ and denote by $\eta$ the tangent of $\gamma$ in zero. By the induction hypothesis we have $d=\frac{\nu}{q}$ for some $\nu \geq q+j-1$ and $\gamma(t)=\sum_{i=q}^{\nu-1} a_{i} t^{i / q}$. Let

$$
M_{\gamma, d}:=\left\{\zeta \in\left(T_{\gamma, d} V\right)_{\operatorname{sing}} \cap \mathbb{R}^{3}:\langle\zeta, \eta\rangle=0 \text { and } \zeta \text { is not simple }\right\} .
$$

By Lemma 4.10, $M_{\gamma, d}$ is finite. For $\zeta \in M_{\gamma, d}$ define

$$
\gamma_{\zeta}: t \mapsto \gamma(t)+\zeta t^{d}=\sum_{i=q}^{\nu} a_{i} t^{i / q}, \quad a_{\nu}=\zeta .
$$

Since $\langle\zeta, \eta\rangle=0, \gamma_{\zeta}$ is in standard parametrization. Next let $A(\gamma, \zeta):=T_{\zeta}\left(T_{\gamma, d} V\right) \cup$ $T_{0} V$. Then $\Delta^{A(\gamma, \zeta)}(\gamma, d)=\frac{\mu}{q}$ for some $\mu>\nu \geq q+j-1$ by Lemma 4.8. Hence the set

$$
\mathcal{C}_{j}:=\left\{\left(\gamma_{\zeta}, \Delta^{A(\gamma, \zeta)}(\gamma, d)\right):(\gamma, d) \in \mathcal{C}_{j-1, c}, \zeta \in M_{\gamma, d}\right\}
$$

is finite and all conditions in (5.1) are satisfied. Consequently, the induction step is complete.

Now we claim

$$
\begin{aligned}
& \text { There exists } N \in \mathbb{N} \text { such that for each }(\gamma, d) \in \mathcal{C}_{N} \text { all points in } \\
& \left(T_{\gamma, d} V\right)_{\text {sing }} \cap \mathbb{R}^{3} \text { are simple. }
\end{aligned}
$$

To prove this, we argue by contradiction. If (5.2) does not hold, then $\mathcal{C}_{j, c} \neq \emptyset$ for each $j \in \mathbb{N}$. Now let

$$
A:=T_{0} V \cup \bigcup_{\eta \in M_{1}} T_{\eta}\left(T_{0} V\right) \cup \bigcup_{j \in \mathbb{N}}\left\{T_{\zeta}\left(T_{\gamma, d} V\right):(\gamma, d) \in \mathcal{C}_{j, c}, \zeta \in M_{\gamma, d}\right\} .
$$

Since $A$ is a countable union of analytic sets, we can choose $\xi_{0} \in \mathbb{R}^{3} \backslash A,\left|\xi_{0}\right|=1$, and find real simple curves $\tau_{1}, \ldots, \tau_{m}$ and a zero neighborhood $U$ in $\mathbb{R}^{3}$ such that (4.3) holds. It is no restriction to assume that $\operatorname{tr}\left(\tau_{i}\right)$ and $\operatorname{tr}\left(\tau_{k}\right)$ are disjoint for 
$i \neq k$. Hence we can choose $\delta>1$ so large that there are $R>0$ and $\varepsilon>0$ such that

$$
\Gamma\left(\tau_{j}, \delta, B(0, \varepsilon), R\right) \cap \Gamma\left(\tau_{k}, \delta, B(0, \varepsilon), R\right)=\emptyset, j \neq k .
$$

Next choose $p \in \mathbb{N}$ so large that $p \geq \delta q$. Then fix $(\gamma, d) \in \mathcal{C}_{p, c}$ and $\zeta \in M_{\gamma, d}$. By (5.1) and the choice of $p$ we have $d \geq \frac{q+p}{q}=1+\frac{p}{q}>\delta$. Since $\zeta$ is not a simple point and since $\xi_{0} \in \mathbb{R}^{3} \backslash A \subset \mathbb{R}^{3} \backslash\left(T_{\zeta}\left(T_{\gamma, d} V\right) \cup T_{0} V\right)$, for each zero neighborhood $D$ in $\mathbb{C}^{3}$ and each $\rho>0$ there are at least two branches of $B_{\xi_{0}} \cap \mathbb{R}^{3}$ which have a nonempty intersection with $\Gamma(\gamma, d, \zeta+D, \rho)=\Gamma\left(\gamma_{\zeta}, d, D, \rho\right)$. Since $d>\delta$ this contradicts (5.3) when $D:=B(0, \varepsilon)$ and $\rho:=R$. From this contradiction we obtain that (5.2) holds. Now let $\mathcal{C}:=\bigcup_{j=1}^{N} \mathcal{C}_{j}$.

5.2. Remark. If the variety $V$ in section 5.1 is algebraic, then estimates for the numbers $q, \delta$, and $N$ can be given in terms of the degree $m$ of a polynomial defining $V$. First, the number of possible solution branch curves can be estimated by $m^{3}$. This implies that $\left(\mathrm{m}^{3}\right)$ ! is an upper estimate for $q$. Finally, using Bezout's theorem, it can be shown that a possible choice for $\delta$ is $\delta=m^{4}$. Thus we arrive at the estimate $N \leq\left(m^{3}\right) ! m^{4}$ for the number of steps in the iteration. This estimate is probably very crude.

5.3. Theorem. For an analytic surface $V$ in $\mathbb{C}^{3}$ which contains the origin, the following conditions are equivalent:

(a) $V$ satisfies $\mathrm{PL}_{\mathrm{loc}}(0)$.

(b) $V$ is hyperbolic in conoids at the origin.

(c) $T_{0} V$ satisfies $\mathrm{PL}_{\mathrm{loc}}(0)$, there is $\zeta \in S^{2}$ such that for $\gamma_{\zeta}: t \mapsto t \zeta, t>0$, and each $\eta \in\left(T_{0} V\right)_{\mathrm{reg}} \cap S^{2}, V$ is $\left(\gamma_{\zeta}, 1\right)$-hyperbolic at $\eta-\zeta \in T_{\gamma_{\zeta}, 1} V$, and for each $(\gamma, d) \in \mathcal{C}(\mathcal{C}$ as in section 5.1) the following conditions hold:

(i) $T_{\gamma, d} V$ satisfies $\mathrm{PL}_{\text {loc }}(\eta)$ at each $\eta \in T_{\gamma, d} V \cap \mathbb{R}^{3}$.

(ii) $V$ is $(\gamma, d)$-hyperbolic at each $\eta \in\left(T_{\gamma, d} V\right)_{\operatorname{reg}} \cap \mathbb{R}^{3}$.

The proof of Theorem 5.3 will be given at the end of this section since we have to introduce more notations and to prove several auxiliary results before.

Remark. In Theorem 7.3 below it is shown that the hyperbolicity conditions in Theorem 5.3(c) follow from the $\mathrm{PL}_{\text {loc }}$-conditions provided that for each $(\gamma, d) \in \mathcal{C}$ the varieties $T_{\gamma, d} V$ as well as $T_{0} V$ have multiplicity one (see Definition 2.10).

5.4. Remark. Let us now indicate how Lemma 3.20 enables us to decide in finitely many steps whether condition (c) of Theorem 5.3 holds: To do so, fix an arbitrary $\zeta \in S^{2}$. We claim that condition (c) of Theorem 5.3 holds if and only if the following conditions hold:

(1) $T_{0} V$ does not admit an elliptic component, i.e., an irreducible component $W$ satisfying $W \cap \mathbb{R}^{3}=\{0\}$,

(2) if $(\gamma, d) \in \mathcal{C} \cup\left\{\left(\gamma_{\zeta}, 1\right)\right\}$, then condition (c)(ii) of Theorem 5.3 holds for $(\gamma, d)$,

(3) $T_{0} V$ is locally hyperbolic at each $\eta \in\left(T_{0} V\right)_{\text {sing }} \cap S^{2}$,

(4) if $(\gamma, d) \in \mathcal{C}$, then $T_{\gamma, d} V$ is locally hyperbolic at each $\eta \in\left(T_{\gamma, d} V\right)_{\text {sing }} \cap E$, where $E \subset \mathbb{R}^{3}$ is the orthogonal plane to the tangent to $\gamma$ at the origin.

To prove equivalence, let us first assume that 5.3(c) is satisfied. Then (1) is immediate by [26], Theorem 3.13. By Proposition 3.12, condition 5.3(c)(ii) is necessary for each pair $(\gamma, d)$ where $\gamma$ is a real simple curve and $d \geq 1$. Hence $(2)$ holds. Conditions (3) and (4) follow from Lemma 3.17. 
To prove the converse, let (1)-(4) be satisfied. Then 5.3(c)(ii) obviously holds. We claim that for each pair $(\gamma, d)$, this condition implies 5.3(c)(i) when $\eta$ is restricted to $\left(T_{\gamma, d}\right)_{\text {reg }} \cap \mathbb{R}^{3}$. To see this, fix $\eta \in\left(T_{\gamma, d}\right)_{\text {reg }} \cap \mathbb{R}^{3}$ and let $\pi$ be a noncharacteristic projection for $T_{\gamma, d} V$ at $\eta$. Then there is a conoid $\Gamma:=\Gamma(\gamma, d, B(\eta, 2 r), r)$, $r>0$, such that $z \in V \cap \Gamma$ is real whenever $\pi(z)$ is real. Let $w \in T_{\gamma, d} V \cap B(\eta, r)$ satisfy $\pi(w) \in \mathbb{R}^{3}$. Then there is a sequence $\left(z_{j}\right)_{j \in \mathbb{N}}$ in $V \cap \Gamma$ with $\lim _{j \rightarrow \infty} z_{j}=w$ and $\pi\left(z_{j}\right) \in \mathbb{R}^{3}$. The hyperbolicity of $V$ in $\Gamma$ immediately implies $z_{j} \in \mathbb{R}^{3}$ for each $j$ and finally $w \in \mathbb{R}^{3}$. This proves the intermediate claim.

Applying this claim, we get 5.3(c)(i) for each $\eta \in\left(T_{\gamma, d} V\right)_{\text {reg }} \cap \mathbb{R}^{3}$. For the singular points, 5.3(c)(i) follows from (4) and the shift invariance of $T_{\gamma, d} V$ (see Theorem 2.7(c)). The same argument shows the part of the conditions in 5.3(c) which pertains to $\zeta$, but only for the particular $\zeta$ chosen at the beginning of this remark. However, since $T_{\gamma_{\zeta}, 1} V=T_{0} V-\zeta$ by Theorem 2.7(b), this condition is independent of the choice of $\zeta$. We have established the equivalence of 5.3(c) with (1)-(4).

This shows that 5.3(c) can be checked in finitely many steps, since (1), (3), and (4) consist of a finite number of conditions, while Lemma 3.20 enables us reduce (2) to a finite number of conditions.

5.5. Definition. Let $V \subset \mathbb{C}^{n}$ be an analytic variety of pure dimension $k$ which contains the origin, let $\gamma$ be a real simple curve in $\mathbb{C}^{n}, d \geq 1, R>0, D$ an open set in $\mathbb{C}^{n}$, and let $\Gamma:=\Gamma(\gamma, d, D, R)$ be a conoid. We say that $V$ satisfies the condition $\operatorname{PL}(V, \Gamma)$ if the following holds: For each compact set $K \subset D$ there exist $A_{0}, r_{0}>0$ such that each $u \in \operatorname{PSH}(V \cap \Gamma)$ which satisfies

( $\alpha) u(z) \leq|z|^{d}, \quad z \in V \cap \Gamma$,

(в) $u(z) \leq 0, \quad z \in V \cap \Gamma \cap \mathbb{R}^{n}$,

also satisfies

$(\gamma) u(z) \leq A_{0}|\operatorname{Im} z|, \quad z \in V \cap \Gamma(\gamma, d, K, R) \cap B\left(0, r_{0}\right)$.

5.6. Lemma. Let $V$ be an analytic variety of pure dimension $k$ in $\mathbb{C}^{n}$ which contains the origin. Let $\gamma$ be a real simple curve, $0<R \leq 1,1 \leq d<\infty$, and $D$ a bounded open set in $\mathbb{C}^{n}$. Assume that $R^{d-1} \sup \{|z|: z \in D\}<1 / 2$. Then the following assertions hold:

(a) If $V$ satisfies $\operatorname{PL}(V, \Gamma(\gamma, d, D, R))$, then for each open subset $G$ of $D$ and $0<r \leq R, V$ satisfies $\operatorname{PL}(V, \Gamma(\gamma, d, G, r))$.

(b) If for each $\xi \in D \cap T_{\gamma, d} V \cap \mathbb{R}^{n}$ there exist an open neighborhood $D_{\xi} \subset$ $D$ of $\xi$ and $R_{\xi}>0$ such that $V$ satisfies $\operatorname{PL}\left(V, \Gamma_{\xi}\right)$ for the conoid $\Gamma_{\xi}:=$ $\Gamma\left(\gamma, d, D_{\xi}, R_{\xi}\right)$, then $V$ satisfies $\operatorname{PL}(V, \Gamma(\gamma, d, D, R))$ for each $R>0$.

Proof. (a) Fix an open set $G$ in $D$, a compact set $K$ in $G$ and $0<r \leq R$. Then let $\Gamma_{D}:=\Gamma(\gamma, d, D, R)$ and $\Gamma_{X}:=\Gamma(\gamma, d, X, r)$ for $X=G$ and $X=K$. Furthermore, let $L:=\sup _{z \in D}|z|$ and note that by hypothesis there exists $0<b<1 / 2$ such that $R^{d-1} L<b$, hence

$$
t^{d} L \leq b t \quad \text { for } \quad t \in[0, R] .
$$

Since $V$ satisfies $\operatorname{PL}\left(V, \Gamma_{D}\right)$, there exist $A_{0}, r_{0}>0$, depending on $K$, so that the conclusion of Definition 5.5 holds for $\Gamma=\Gamma_{D}$. It is no restriction to assume $\frac{1}{2} \leq\left|\frac{\gamma(t)}{t}\right| \leq 2$ for all $\left.t \in\right] 0, r[$. Now choose $\eta>0$ such that

$$
K+B(0,2 \eta) \subset G \text { and } \eta<1
$$


and let $\rho_{0}:=\min \left(r, r_{0}\right)$. To prove that $V$ satisfies $\operatorname{PL}\left(V, \Gamma_{G}\right)$, fix $u \in \operatorname{PSH}\left(V \cap \Gamma_{G}\right)$ satisfying the estimates $5.5(\alpha)$ and $(\beta)$ for $\Gamma=\Gamma_{G}$. Then choose $0<\alpha<R$ such that for each $z_{0} \in V \cap \Gamma_{K} \cap B\left(0, \rho_{0}\right)$ there are $0<t_{0} \leq \alpha_{1}$ and $\zeta_{0} \in K$ satisfying

$$
z_{0}=\gamma\left(t_{0}\right)+t_{0}^{d} \zeta_{0} \text {. }
$$

Then fix $z_{0}$ and consider the following two cases:

Case 1: $\left|\operatorname{Im} z_{0}\right| \geq \eta t_{0}^{d}$. In this case, condition 5.5( $\left.\alpha\right)$ for $u$ implies

$$
u\left(z_{0}\right) \leq\left|z_{0}\right|^{d}=\left|\gamma\left(t_{0}\right)+t_{0}^{d} \zeta_{0}\right|^{d} \leq t_{0}^{d}\left|\frac{\gamma\left(t_{0}\right)}{t_{0}}+t_{0}^{d-1} \zeta_{0}\right|^{d} \leq(2+L)^{d} \frac{1}{\eta}\left|\operatorname{Im} z_{0}\right| .
$$

Case 2: $\left|\operatorname{Im} z_{0}\right|<\eta t_{0}^{d}$. In this case the choice of $\eta$ implies that each $z \in$ $B\left(\operatorname{Re} z_{0}, \eta t_{0}^{d}\right)$ satisfies

$$
z-\gamma\left(t_{0}\right)=z-\operatorname{Re} z_{0}-i \operatorname{Im} z_{0}+z_{0}-\gamma\left(t_{0}\right) \in t_{0}^{d} B(0,2 \eta)+t_{0}^{d} K \subset t_{0}^{d} G .
$$

Hence we have $B\left(\operatorname{Re} z_{0}, \eta t_{0}^{d}\right) \subset \Gamma_{G}$. Therefore we can define $\varphi: V \rightarrow[-\infty, \infty[$ by

$$
\varphi(z):=\max \left(\frac{\eta}{2(3+b / L)^{d}} u(z)+\eta t_{0}^{d} H\left(\frac{z-\operatorname{Re} z_{0}}{\eta t_{0}^{d}}\right),|\operatorname{Im} z|\right)
$$

for $z \in V \cap B\left(\operatorname{Re} z_{0}, \eta t_{0}^{d}\right)$ and by

$$
\varphi(z):=|\operatorname{Im} z| \text { otherwise, }
$$

where $H$ is the function defined in Lemma 3.2. For $z \in \mathbb{C}^{n}$ satisfying $\left|z-\operatorname{Re} z_{0}\right|=$ $\eta t_{0}^{d}$ we have

$|z| \leq\left|z-\operatorname{Re} z_{0}\right|+\left|\operatorname{Re} z_{0}\right| \leq \eta t_{0}^{d}+\left|\gamma\left(t_{0}\right)\right|+t_{0}^{d}\left|\zeta_{0}\right| \leq\left(\eta t_{0}^{d-1}+2+b / L\right) t_{0}<(3+b / L) t_{0}$ and hence

$$
\frac{\eta}{2(3+b / L)^{d}} u(z)<\frac{\eta}{2} t_{0}^{d}
$$

From this estimate and the properties of the function $H$ it follows as in the proof of Lemma 3.4 that $\varphi$ is plurisubharmonic on $V$. To estimate $\varphi$ on $V \cap \Gamma_{D}$, fix $z \in \Gamma_{D}, z=\gamma(t)+t^{d} \zeta, 0<t<R, \zeta \in D$. Then we have by (5.4)

$$
|z| \geq|\gamma(t)|-t^{d}|\zeta| \geq \frac{1}{2} t-t^{d} L \geq t\left(\frac{1}{2}-b\right)
$$

and consequently

$$
|\operatorname{Im} z|=t^{d}|\operatorname{Im} \zeta| \leq L t^{d} \leq L\left(\frac{1}{2}-b\right)^{-d}|z|^{d} .
$$

For $z \in V \cap B\left(\operatorname{Re} z_{0}, \eta t_{0}^{d}\right)$ this and the estimates 5.5( $\alpha$ ) for $u$ and 3.2(a) for $H$ imply

$$
\varphi(z) \leq \max \left(\frac{\eta}{2(3+b / L)^{d}}|z|^{d}+|\operatorname{Im} z|,|\operatorname{Im} z|\right) \leq\left(\frac{\eta}{2(3+b / L)^{d}}+\frac{L}{(1 / 2-b)^{d}}\right)|z|^{d} .
$$

Now let $M:=\frac{\eta}{2(3+b / L)^{d}}+\frac{L}{(1 / 2-b)^{d}}$. Then we have

$$
\varphi(z) \leq M|z|^{d}, z \in V \cap \Gamma_{D} \text { and } \varphi(z) \leq 0, z \in V \cap \Gamma_{D} \cap \mathbb{R}^{n} .
$$

Since $V$ satisfies $\operatorname{PL}\left(V, \Gamma_{0}\right)$ we conclude from this that

$$
\varphi(z) \leq A_{0} M|\operatorname{Im} z|, z \in V \cap \Gamma_{K} \cap B\left(0, r_{0}\right) .
$$

Now note that the present case implies $z_{0} \in V \cap B\left(\operatorname{Re} z_{0}, \eta t_{0}\right)$. Thus the definition of $\varphi$ and property $3.2(\mathrm{~d})$ of $H$ give

$$
A_{0} M\left|\operatorname{Im} z_{0}\right| \geq \varphi\left(z_{0}\right) \geq \frac{\eta}{2(3+b / L)^{d}} u\left(z_{0}\right)+\eta t_{0} H\left(\frac{i \operatorname{Im} z_{0}}{\eta t_{0}}\right) \geq \frac{\eta}{2(3+b / L)^{d}} u\left(z_{0}\right)
$$


and hence

$$
u\left(z_{0}\right) \leq \frac{2(3+b / L)^{d}}{\eta} A_{0} M\left|\operatorname{Im} z_{0}\right|
$$

Summing up both cases and letting $A_{K}:=\frac{1}{\eta} \max \left(2(3+b / L)^{d} A_{0} M, 2+L\right)$ we proved

$$
u\left(z_{0}\right) \leq A_{K}\left|\operatorname{Im} z_{0}\right| \quad \text { for each } \quad z_{0} \in V \cap \Gamma_{K} \cap B\left(0, \rho_{0}\right) .
$$

Since $K$ was any compact subset of $G$, the proof of part (a) is complete.

(b) Fix $R>0$ and a compact subset $K$ of $D$. Then the present hypothesis implies that for each $\xi \in K \cap T_{\gamma, d} V \cap \mathbb{R}^{n}$ there exists an open neighborhood $D_{\xi}$ of $\xi, D_{\xi} \subset D$, such that $V$ satisfies $\operatorname{PL}\left(V, \Gamma_{\xi}\right)$ for $\Gamma_{\xi}=\Gamma\left(\gamma, d, D_{\xi}, R_{\xi}\right)$, where we may assume $R_{\xi} \leq R$ in view of part (a). For each such $\xi$ choose a compact neighborhood $K_{\xi}$ of $\xi, K_{\xi} \subset D$. Since $K \cap T_{\gamma, d} \cap \mathbb{R}^{n}$ is compact, there exist $K_{j}:=K_{\xi_{j}}, 1 \leq j \leq l$, such that

$$
K \cap T_{\gamma, d} V \cap \mathbb{R}^{n} \subset \bigcup_{j=1}^{l} \stackrel{\circ}{K}_{j}
$$

and $\varepsilon>0$ such that

$$
K \cap T_{\gamma, d} V \cap \mathbb{R}^{n}+B^{n}(0, \varepsilon) \subset \bigcup_{j=1}^{l} \stackrel{\circ}{K}_{j} .
$$

As in Lemma 2.8 it follows that for some $\rho_{0}>0$ and each $0<t \leq \rho_{0}$ we have

$$
K \cap V_{t, d} \cap \mathbb{R}^{n} \subset\left(K \cap T_{\gamma, d} V \cap \mathbb{R}^{n}\right)+B^{n}(0, \varepsilon) \subset \bigcup_{j=1}^{l} \stackrel{\circ}{K}_{j} .
$$

Next choose $\rho>0$ so small that $\rho<\min \left\{\varepsilon, \rho_{0}, R_{\xi_{j}}: 1 \leq j \leq l\right\}$ and such that for each $z \in \Gamma(\gamma, d, K, R) \cap B(0, \rho)$ there exist $0<t \leq \rho_{0}$ and $\zeta \in K$ such that $z=\gamma(t)+t^{d} \zeta$. Then we claim that there exists $\delta>0$ such that

$$
V \cap B^{n}(0, \rho) \cap \bigcup_{0<t \leq \rho_{0}}\left(\gamma(t)+t^{d} K_{\delta}\right) \subset \bigcup_{j=1}^{l} \Gamma\left(\gamma, d, \stackrel{\circ}{K_{j}}, R_{\xi_{j}}\right),
$$

where

$$
K_{\delta}:=\{z \in K:|\operatorname{Im} z| \leq \delta\} .
$$

To prove (5.6), assume that it does not hold. Then there exists a sequence $\left(z_{\nu}\right)_{\nu \in \mathbb{N}}$ satisfying $z_{\nu}=t_{\nu}+t_{\nu}^{d} \zeta_{\nu}, \quad z_{\nu} \in V \cap B^{n}(0, \rho), \quad \zeta_{\nu} \in K_{1 / \nu}$ but $z_{\nu} \notin$ $\bigcup_{j=1}^{l} \Gamma\left(\gamma, d, K_{j}, R_{\xi_{j}}\right)$. Since $K$ is compact, we may assume that $\left(\zeta_{\nu}\right)_{\nu \in \mathbb{N}}$ converges to some $\zeta \in K \cap \mathbb{R}^{n}$, otherwise we pass to a suitable subsequence. Then consider two cases.

Case 1: $\lim _{\nu \rightarrow \infty} t_{\nu}=0$. Then $z_{\nu} \in V_{t_{\nu}, d}$ implies $\zeta \in K \cap \mathbb{R}^{n} \cap T_{\gamma, d} V$. From this and (5.5) we get that $\zeta \in \stackrel{\circ}{K}_{j}$ for some $j, 1 \leq j \leq l$. Hence there exists $\nu_{0} \in \mathbb{N}$ such that $\zeta_{\nu_{0}} \in \stackrel{\circ}{K_{j}}$ and consequently $z_{\nu_{0}} \in \Gamma\left(\gamma, d, \stackrel{\circ}{K_{j}}, R_{\xi_{j}}\right)$, in contradiction to the choice of $\left(z_{\nu}\right)_{\nu \in \mathbb{N}}$.

Case 2: $\left(t_{\nu}\right)_{\nu \in \mathbb{N}}$ does not tend to zero. By passing to a subsequence, we may assume that $\left(t_{\nu}\right)_{\nu \in \mathbb{N}}$ converges to some $\left.\left.t_{0} \in\right] 0, \zeta_{0}\right]$. Then $z_{\nu}=\gamma\left(t_{\nu}\right)+t_{\nu}^{d} \zeta_{\nu}$ converges to $z_{0}=\gamma\left(t_{0}\right)+t_{0}^{d} \zeta$. This shows $\zeta \in V_{t_{0}, d} \cap K \cap \mathbb{R}^{n}$. Hence Definition 5.5 implies the existence of $j, 1 \leq j \leq l$, such that $\zeta \in \stackrel{\circ}{K}_{j}$. As in Case 1 this implies the existence 
of $z_{\nu_{0}} \in \Gamma\left(\gamma, d, \stackrel{\circ}{K_{j}}, R_{\xi_{j}}\right)$, contradicting our choice of the sequence $\left(z_{\nu}\right)_{\nu \in \mathbb{N}}$. From these contradictions we conclude that (5.6) holds.

To complete the proof now let $u \in \operatorname{PSH}(V \cap \Gamma)$ be given and assume that $u$ satisfies $(\alpha)$ and $(\beta)$ of Definition 5.5. If $z \in V \cap \Gamma(\gamma, d, K, \rho) \cap B(0, \rho)$ and $z=\gamma(t)+t^{d} \zeta$, where $|\operatorname{Im} \zeta| \geq \delta$, then we get

$$
|\operatorname{Im} z|=|t|^{d}|\operatorname{Im} \zeta| \geq|t|^{d} \delta \geq\left(\frac{|z|}{2}\right)^{d} \delta
$$

(possibly, $\rho$ has to be decreased). From this and the condition $(\alpha)$ for $u$, we get

$$
u(z) \leq|z|^{d} \leq \frac{2^{d}}{\delta}|\operatorname{Im} z| .
$$

If $|\operatorname{Im} \zeta|<\delta$, then (5.6) implies that there is some $j, 1 \leq j \leq l$, such that

$$
z \in \Gamma\left(\gamma, d, \stackrel{\circ}{K}_{j}, R_{\xi_{j}}\right) \subset \Gamma\left(\gamma, d, D_{\xi_{j}}, R_{\xi_{j}}\right)=: \Gamma_{\xi_{j}} .
$$

Since $\left.u\right|_{V \cap \Gamma_{\xi_{j}}}$ obviously satisfies the conditions $(\alpha)$ and $(\beta)$ of Definition 5.5, the hypothesis implies the existence of $A_{j}>0$ and $v_{j}>0$ such that

$$
u(z) \leq A_{j}|\operatorname{Im} z| \text { if } z \in V \cap \Gamma\left(\gamma, d, K_{j}, R_{\xi_{j}}\right) \cap B\left(0, r_{j}\right) .
$$

Now let $A:=\max \left\{\frac{2^{d}}{\delta}, A_{j}: 1 \leq j \leq l\right\}$ and $r:=\min \left\{\rho, r_{j}: 1 \leq j \leq l\right\}$. Then

$$
u(\zeta) \leq A|\operatorname{Im} \zeta|, z \in V \cap \Gamma(\gamma, d, K, R) \cap B(0, r) .
$$

Since $K$ was an arbitrary compact subset of $D$, the proof is complete.

Using Lemma 5.6 and standard arguments we get

5.7. Lemma. Let $V$ be an analytic variety of pure dimension $k$ in $\mathbb{C}^{n}$, let $\gamma$ be a real simple curve and $d \geq 1$. If $V$ is $(\gamma, d)$-hyperbolic in $\xi \in T_{\gamma, d} V \cap \mathbb{R}^{n}$, then there exists a zero-neighborhood $G$ in $\mathbb{C}^{n}$ such that $V$ satisfies $\operatorname{PL}(V, \Gamma(\gamma, d, \xi+G, r))$ for each $r>0$.

Proof. Since $V$ is $(\gamma, d)$-hyperbolic in $\xi$, there exist an open neighborhood $D$ of $\xi, r_{0}>0$, and a projection $\pi$ in $\mathbb{C}^{n}$ which is noncharacteristic for $T_{\gamma, d} V$ at $\xi$ such that $z \in V \cap \Gamma\left(\gamma, d, D, r_{0}\right)$ is real whenever $\pi(z)$ is real. After a real linear change of variables we may assume that $\mathbb{C}^{n}=\mathbb{C}^{k} \times \mathbb{C}^{n-k}$ and $\pi(z)=\left(z^{\prime}, 0\right)$ for $z=\left(z^{\prime}, z^{\prime \prime}\right) \in \mathbb{C}^{k} \times \mathbb{C}^{n-k}$. Since $\pi$ is noncharacteristic for $T_{\gamma, d} V$ at $\xi$, by Lemma 3.10 we get $\varepsilon^{\prime}>0$ and $0<\varepsilon_{1}^{\prime \prime}<\varepsilon^{\prime \prime}$ such that $B^{k}\left(\xi^{\prime}, \varepsilon^{\prime}\right) \times B^{n-k}\left(\xi^{\prime \prime}, \varepsilon^{\prime \prime}\right) \subset D$ and

$$
B^{k}\left(\xi^{\prime}, \varepsilon^{\prime}\right) \times B^{n-k}\left(\xi^{\prime \prime}, \varepsilon^{\prime \prime}\right) \cap T_{\gamma, d} V \subset B^{k}\left(\xi^{\prime}, \varepsilon^{\prime}\right) \times B^{n-k}\left(\xi^{\prime \prime}, \varepsilon_{1}^{\prime \prime}\right) \cap T_{\gamma, d} V .
$$

Now choose $0<\varepsilon_{2}^{\prime}<\varepsilon^{\prime}$ and $\varepsilon_{1}^{\prime \prime}<\varepsilon_{2}^{\prime \prime}<\varepsilon^{\prime \prime}$, let

$$
G:=B^{k}\left(0, \varepsilon_{2}^{\prime}\right) \times B^{n-k}\left(0, \varepsilon_{2}^{\prime \prime}\right) \text { and } Q:=\xi+\bar{G},
$$

and choose $0<\eta_{0}<\varepsilon_{2}^{\prime \prime}-\varepsilon_{1}^{\prime \prime}$ so small that $Q+B^{n}\left(0, \eta_{0}\right) \subset B^{k}\left(\xi^{\prime}, \varepsilon^{\prime}\right) \times B^{n-k}\left(\xi^{\prime \prime}, \varepsilon^{\prime \prime}\right)$. Next note that by Lemma 2.8 and Theorem 2.7(d) there exists $0<r_{1} \leq r_{0}$ such that for $\left.t \in] 0, r_{1}\right]$ we have

$V_{t, d} \cap Q \subset T_{\gamma, d} V \cap Q+B^{n}\left(0, \eta_{0}\right) \subset T_{\gamma, d} V \cap\left(\overline{B^{k}\left(\xi^{\prime}, \varepsilon_{2}^{\prime}\right) \times B^{n-k}\left(\xi^{\prime \prime}, \varepsilon_{1}^{\prime \prime}\right)}\right)+B^{n}\left(0, \eta_{0}\right)$.

We now claim that for $0<t<r_{1}$ the map

$$
\tilde{\pi}:\left(\gamma(t)+t^{d}(\xi+G)\right) \cap V \rightarrow(\gamma(t))^{\prime}+t^{d}\left(\xi^{\prime}+B^{k}\left(0, \varepsilon_{2}^{\prime}\right)\right), \tilde{\pi}\left(z^{\prime}, z^{\prime \prime}\right):=z^{\prime}
$$


is proper. To prove this claim, fix $0<\sigma<\varepsilon_{2}^{\prime}$ and $z \in\left(\gamma(t)+t^{d}(\xi+G)\right) \cap V$ and assume that $\tilde{\pi}(z)=z^{\prime} \in(\gamma(t))^{\prime}+t^{d} \overline{B^{k}\left(\xi^{\prime}, \sigma\right)}$. Then $z=\gamma(t)+t^{d}(\xi+w)$, where $\xi+w \in V_{t, d} \cap Q$. By (5.7) this means that $\xi+w=\zeta+h$ for suitable $\zeta \in \overline{B^{k}\left(\xi^{\prime}, \varepsilon_{2}^{\prime}\right) \times B^{n-k}\left(\xi^{\prime}, \varepsilon_{1}^{\prime \prime}\right)}$ and $h \in B^{n}\left(0, \eta_{0}\right)$. From this we get

$$
\left|w^{\prime \prime}\right| \leq\left|\zeta^{\prime \prime}-\xi^{\prime \prime}\right|+\left|h^{\prime \prime}\right| \leq \varepsilon_{1}^{\prime \prime}+\eta_{0}
$$

This shows that

$$
z=\left(z^{\prime}, z^{\prime \prime}\right) \in\left(\gamma(t)+t^{d} \overline{B^{k}\left(\xi^{\prime}, \sigma\right) \times B^{n-k}\left(\xi^{\prime \prime}, \varepsilon_{1}^{\prime \prime}+\eta_{0}\right)}\right) \cap V .
$$

Since the latter set is compact in $\left(\gamma(t)+t^{d}(\xi+G)\right) \cap V$, the claim is proved.

Now let $\Gamma:=\Gamma\left(\gamma, d, \xi+G, r_{1}\right)$. Shrinking $r_{1}$ if necessary, we can find $C>0$ such that for $\left.t \in] 0, r_{1}\right]$ and $z \in \gamma(t)+t^{d}(\xi+G)$ we have

$$
|z| \leq|\gamma(t)|+t^{d}\left(|\xi|+\sqrt{2} \max \left(\varepsilon_{2}^{\prime}, \varepsilon_{2}^{\prime \prime}\right)\right) \leq C t .
$$

To show that $V$ satisfies $\operatorname{PL}(V, \Gamma)$, fix $u \in \operatorname{PSH}(V \cap \Gamma)$ satisfying the conditions $5.5(\alpha)$ and $(\beta)$ and fix a compact subset $K$ of $G$. Obviously, it is no restriction to assume that $K=\overline{B^{k}\left(0, \sigma^{\prime}\right)} \times B^{n-k}\left(0, \sigma^{\prime \prime}\right)$, where $0<\sigma^{\prime}<\varepsilon_{2}^{\prime}$ and $0<\sigma^{\prime \prime}<\varepsilon_{2}^{\prime \prime}$. Next choose $\delta>0$ so small that $\sigma^{\prime}+2 \delta<\varepsilon_{2}^{\prime}$. Then (5.8) implies that for $0<t<r_{1}$ the map

$$
\begin{aligned}
\tilde{\pi}:\left(\gamma(t)+t^{d}\left(B^{k}\left(\xi^{\prime}, \sigma^{\prime}+2 \delta\right) \times B^{n-k}\right.\right. & \left.\left.\left(\xi^{\prime \prime}, \varepsilon_{2}^{\prime \prime}\right)\right)\right) \cap V \\
& \rightarrow(\gamma(t))^{\prime}+t^{d} B^{k}\left(\xi^{\prime}, \sigma^{\prime}+2 \delta\right), \tilde{\pi}\left(z^{\prime}, z^{\prime \prime}\right)=z^{\prime}
\end{aligned}
$$

is proper. Then fix $\eta^{\prime} \in \mathbb{R}^{k},\left|\eta^{\prime}\right| \leq \sigma^{\prime}$ and define $\varphi: B^{k}(0,1) \rightarrow[-\infty, \infty[$ by

$$
\begin{array}{r}
\varphi(\lambda):=t^{-d} \max \left\{u(z): z \in\left(\gamma(t)+t^{d}\left(\xi+\left(\eta^{\prime}, 0\right)+B^{k}(0,2 \delta) \times B^{n-k}\left(0, \varepsilon_{2}^{\prime \prime}\right)\right)\right) \cap V,\right. \\
\left.\tilde{\pi}(z)=(\gamma(t))^{\prime}+t^{d}\left(\xi^{\prime}+\eta^{\prime}+2 \delta \lambda\right)\right\} .
\end{array}
$$

Since $\tilde{\pi}$ is proper, it follows from Chirka [14, Theorem 3.7, and Hörmander [17, Lemma 4.4 , that $\varphi$ is plurisubharmonic on $B^{k}(0,1)$. The estimate $5.5(\alpha)$ for $u$ together with (5.9) implies

$$
\varphi(\lambda) \leq C^{d}, \lambda \in B^{k}(0,1),
$$

while the $(\gamma, d)$-hyperbolicity and $(\beta)$ imply

$$
\varphi(\lambda) \leq 0, \lambda \in B^{k}(0,1) \cap \mathbb{R}^{k} .
$$

From these two estimates and a standard application of a classical estimate (see Nevanlinna [28], 38, or Braun, Meise, and Vogt [12], Lemma 4.1), we get

$$
\varphi(\lambda) \leq C^{d} \frac{8}{\pi}|\operatorname{Im} \lambda|, \lambda \in \overline{B^{k}\left(0, \frac{1}{2}\right)} .
$$

By the definition of $\varphi$, this estimate implies that for $z=\gamma(t)+t^{d}\left(\xi+\left(\eta^{\prime}, 0\right)+w\right) \in V$ with $w \in B^{k}(0, \delta) \times B^{n-k}\left(0, \varepsilon_{2}^{\prime \prime}\right)$ we have

$$
u(z) \leq t^{d} \varphi\left(\frac{w^{\prime}}{2 \delta}\right) \leq C^{d} \frac{8}{\pi} t^{d}\left|\operatorname{Im} \frac{w^{\prime}}{2 \delta}\right| \leq \frac{4 C^{d}}{\pi \delta} t^{d}|\operatorname{Im} w|=\frac{4 C^{d}}{\pi \delta}|\operatorname{Im} z| .
$$

Next we show that this statement implies

$$
u(z) \leq \frac{2 C^{d}}{\delta}|\operatorname{Im} z| \text { for all } z \in \Gamma\left(\gamma, d, \xi+K, r_{1}\right) \cap V .
$$


To prove this, fix $z=\gamma(t)+t^{d}(\xi+w) \in \Gamma\left(\gamma, d, \xi+K, r_{1}\right) \cap V$, where $w \in K$. If $|\operatorname{Im} w| \geq \delta$, then $|\operatorname{Im} z|=t^{d}|\operatorname{Im} w| \geq t^{d} \delta$. Since $u$ satisfies the condition $5.5(\alpha)$, (5.9) implies

$$
u(z) \leq|z|^{d} \leq C^{d} t^{d} \leq \frac{2 C^{d}}{\delta}|\operatorname{Im} z| .
$$

If $|\operatorname{Im} w|<\delta$, then $\eta^{\prime}:=\operatorname{Re} w^{\prime}$ is in $B^{k}\left(0, \sigma^{\prime}\right)$ and we have $w \in\left(\eta^{\prime}, 0\right)+B^{k}(0, \delta) \times$ $B^{n-k}\left(0, \varepsilon_{2}^{\prime \prime}\right)$. Therefore, the estimate above gives

$$
u(z) \leq \frac{4 C^{d}}{\pi \delta}|\operatorname{Im} z| \leq \frac{2 C^{d}}{\delta}|\operatorname{Im} z| .
$$

Hence we proved (5.10). Since $K$ was an arbitrary compact subset of $G$, we proved that $V$ satisfies $\operatorname{PL}\left(V, \Gamma\left(\gamma, d, \xi+G, r_{1}\right)\right)$. Now the lemma follows from Lemma $5.6(\mathrm{a})$.

Proof of Lemma 3.19. By Lemma 5.7 there exist a zero neighborhood $G$ in $\mathbb{C}^{n}$ and $\rho>0$ such that $V$ satisfies $\operatorname{PL}(V, \Gamma(\gamma, d, \kappa+G, \rho))$. Now note that one can prove a modified version of Lemma 3.4 under the weaker hypothesis that $V$ satisfies $\mathrm{PL}(V, \Gamma(\gamma, d, \kappa+G, \rho))$ instead of $\mathrm{PL}_{\mathrm{loc}}(0)$. The modification in the conclusion is that the open set $D$ and the all quantifier over it get replaced by the open set $\kappa+G$. Using this conclusion, it follows as in the proof of Proposition 3.12 that $V$ is $(\gamma, d)$-hyperbolic with respect to each projection $\pi$ which is noncharacteristic for $T_{\gamma, d} V$ at $\kappa$.

In the remaining part of this section, $\Gamma^{\prime}$ will denote a conoid in $\mathbb{C}^{2}$.

5.8. Lemma. Let $V$ be an analytic surface in $\mathbb{C}^{3}$ which contains the origin, let $\gamma$ be a real simple curve which is in standard parametrization with respect to the canonical basis of $\mathbb{R}^{3}$, and let $d \geq 1$. Assume further that $0 \in T_{\gamma, d} V$ and $\pi:\left(z_{1}, z_{2}, z_{3}\right) \mapsto$ $\left(z_{1}, z_{2}, 0\right)$ is noncharacteristic for $T_{\gamma, d} V$ at 0 . Then for each zero neighborhood $D$ in $\mathbb{C}^{3}$ there are $\varepsilon>0, \delta>0$, and $\rho_{1}>0$ such that $G:=B(0, \varepsilon)^{2} \times B(0, \delta)$ is contained in $D$ and

$$
\pi: V \cap \Gamma\left(\gamma, d, G, \rho_{1}\right) \rightarrow \Gamma^{\prime}\left(\pi \circ \gamma, d, B(0, \varepsilon)^{2}, \rho_{1}\right)
$$

is proper.

Proof. Since $\pi$ is noncharacteristic for $T_{\gamma, d} V$ at 0 , we can apply Lemma 3.10 to find $\varepsilon_{1}>0$ and $0<\delta_{0}<\delta_{1}$ such that $G_{0}:=B\left(0, \varepsilon_{1}\right)^{2} \times B\left(0, \delta_{1}\right)$ is contained in $D$ and such that

$$
T_{\gamma, d} V \cap G_{0} \subset T_{\gamma, d} V \cap B\left(0, \varepsilon_{1}\right)^{2} \times B\left(0, \delta_{0}\right) .
$$

Then fix $0<\varepsilon<\varepsilon_{1}$ and $\delta_{0}<\delta<\delta_{2}<\delta_{1}$ and choose $0<\eta<\min \left(\delta_{1}-\delta_{2}, \delta-\delta_{0}\right)$. We claim that for suitable $r>0$

$$
V \cap \Gamma\left(\gamma, d, B(0, \varepsilon)^{2} \times B\left(0, \delta_{2}\right), r\right) \subset V \cap \Gamma\left(\gamma, d, B(0, \varepsilon)^{2} \times B(0, \delta), r\right) .
$$

To prove (5.12) let

$$
K:=\overline{B(0, \varepsilon)^{2} \times B\left(0, \delta_{2}\right)} \quad \text { and } \quad G_{1}:=K \cap T_{\gamma, d} V+B(0, \eta) .
$$

Then it follows from Lemma 2.8 that there exists $0<r$ such that $K \cap V_{t, d} \subset G_{1}$ for all $t \in] 0, r$. To derive (5.12) from this, fix $z=\gamma(t)+t^{d} \zeta \in V$, where $\left.t \in\right] 0, r[$ and $\zeta \in B(0, \varepsilon)^{2} \times B\left(0, \delta_{2}\right)$. This implies $\zeta \in V_{t, d}$. Therefore there exists $w \in K \cap T_{\gamma, d} V$ such that $|\zeta-w|<\eta$. From this we get

$$
\left|w_{3}\right|<\left|\zeta_{3}\right|+\eta<\delta_{2}+\eta<\delta_{1} .
$$


By (5.11) this implies $\left|w_{3}\right|<\delta_{0}$ and consequently

$$
\left|\zeta_{3}\right|<\left|w_{3}\right|+\eta<\delta_{0}+\eta<\delta .
$$

Hence we have $z \in V \cap \Gamma\left(\gamma, \delta, B(0, \varepsilon)^{2} \times B(0, \delta), r\right)$ and (5.12) holds.

To derive the statement of the lemma from (5.12), fix a compact set $Q$ in $\Gamma^{\prime}\left(\pi \circ \gamma, d, B(0, \varepsilon)^{2}, r\right)$ and let

$$
L:=\left\{z \in V \cap \Gamma\left(\gamma, d, B(0, \varepsilon)^{2} \times B(0, \delta), r\right): \pi(z) \in Q\right\} .
$$

To show that $L$ is compact, let

$$
U_{k}:=\bigcup\left\{\pi \circ \gamma(t)+t^{d} B\left(0, \frac{k}{k+1} \varepsilon\right)^{2}: t \in\right] \frac{r}{k+1}, \frac{k r}{k+1}[\} .
$$

Then $\left(U_{k}\right)_{k \in \mathbb{N}}$ is an increasing sequence of open sets satisfying

$$
\bigcup_{k \in \mathbb{N}} U_{k}=\Gamma^{\prime}\left(\pi \circ \gamma, d, B(0, \varepsilon)^{2}, r\right) .
$$

Since $Q$ is compact, there is $k \in \mathbb{N}$ such that $Q \subset U_{k}$. Now fix any sequence $\left(z_{j}\right)_{j \in \mathbb{N}}$ in $L$. Since $L$ is bounded in $\mathbb{C}^{3},\left(z_{j}\right)_{j \in \mathbb{N}}$ contains a subsequence which converges in $\mathbb{C}^{3}$. Hence we may assume that $\left(z_{j}\right)_{j \in \mathbb{N}}$ converges to some $\zeta \in \bar{L}$. Since the sequence $\left(z_{j}^{\prime}\right)_{j \in \mathbb{N}}$ is in $Q \subset U_{k}$, we can choose a sequence $\left(t_{j}\right)_{j \in \mathbb{N}}$ in $] \frac{r}{k+1}, \frac{k r}{k+1}[$ and $\left(w_{j}\right)_{j \in \mathbb{N}} \in B\left(0, \frac{k}{k+1} \varepsilon\right)^{2} \times B(0, \delta)$ such that

$$
z_{j}=\gamma\left(t_{j}\right)+t_{j}^{d} w_{j}, \quad j \in \mathbb{N} .
$$

Passing to suitable subsequences we may assume that $\left(t_{j}\right)_{j \in \mathbb{N}}$ converges to $t_{0} \in$ $\left[\frac{r}{k+1} \frac{k r}{k+1}\right]$ and that $\left(w_{j}\right)_{j \in \mathbb{N}}$ converges to $w_{0} \in \overline{B\left(0, \frac{k}{k+1} \varepsilon\right)^{2} \times B(0, \delta)} \subset B(0, \varepsilon)^{2} \times$ $B\left(0, \delta_{2}\right)$. Since

$$
\zeta=\gamma\left(t_{0}\right)+t_{0}^{d} w_{0}
$$

is also in $V$, we have $\zeta \in V \cap \Gamma\left(\gamma, d, B(0, \varepsilon)^{2} \times B\left(0, \delta_{2}\right), r\right)$. By (5.12) this implies $\zeta \in V \cap \Gamma\left(\gamma, d, B(0, \varepsilon)^{2} \times B(0, \delta), r\right)$ and hence $\zeta \in L$. Since $Q$ was any compact set in $\Gamma^{\prime}\left(\pi \circ \gamma, d, B(0, \varepsilon)^{2}, r\right)$, the lemma is proved with $G:=B(0, \varepsilon)^{2} \times B(0, \delta)$ and $\rho_{1}=r$

5.9. Lemma. Let $V$ be an analytic surface in $\mathbb{C}^{3}$ which contains the origin, let $\gamma$ be a real simple curve, $d \geq 1$, and let $\eta \in T_{\gamma, d} V \cap \mathbb{R}^{3}$ be a simple point of $T_{\gamma, d} V$. Assume that $T_{\gamma, d} V$ is locally hyperbolic at $\eta$ and that there is an open neighborhood $U$ of $\eta$ such that $V$ is $(\gamma, d)$-hyperbolic at each $\zeta \in\left(T_{\gamma, d} V\right)_{\text {reg }} \cap \mathbb{R}^{3} \cap U$. Then $V$ is $(\gamma, d)$-hyperbolic at $\eta$.

Proof. Since $\eta$ is a simple point of $T_{\gamma, d} V$ there are $\xi \in \mathbb{R}^{3} \backslash\left(T_{\eta}\left(T_{\gamma, d} V\right) \cup T_{0} V\right)$, an absolutely convex zero neighborhood $D_{0} \subset U$, and $\rho_{1}>0$ such that

$$
B_{\xi} \cap \mathbb{R}^{3} \cap \Gamma\left(\gamma, d, \eta+D_{0}, \rho_{1}\right)
$$

contains at most one branch $S$ of $B_{\xi} \cap \mathbb{R}^{3}$. Let $\zeta_{1}$ be the tangent vector of $\gamma$ at the origin, choose $\zeta_{2} \in \mathbb{R}^{3}$ such that $\mathfrak{X}:=\left(\zeta_{1}, \zeta_{2}, \xi\right)$ is a basis of $\mathbb{R}^{3}$, and assume that $\gamma=\left(\gamma_{1}, \gamma_{2}, \gamma_{3}\right)$ is in standard parametrization with respect to $\mathfrak{X}$. It is no restriction to assume that $\mathfrak{X}$ is the standard basis of $\mathbb{R}^{3}$. Denote by $\pi$ the projection along $\xi$ on the plane spanned by $\zeta_{1}$ and $\zeta_{2}$. Since $\xi \notin T_{\eta}\left(T_{\gamma, d} V\right), \pi$ is noncharacteristic for $T_{\gamma, d} V$ at $\eta$. 
To simplify the notation, assume from now on that $\eta=0$. By hypothesis $T_{\gamma, d} V$ is locally hyperbolic at $\eta=0$. We claim that it is locally hyperbolic for the given projection $\pi$. To prove this claim, note that

$$
\left[T_{\gamma, d} V\right]_{0}=\bigcup_{j=1}^{m}\left[W_{j}\right]_{0},
$$

where $W_{j}$ is a complex manifold for which $W_{j} \cap \mathbb{R}^{3}$ satisfies the dimension condition at each real point near the origin. This follows for $d>1$ from Theorem 2.7(c) in connection with Proposition 3.16 and for $d=1$ from Theorem 2.7(b) together with Braun [5], Corollary 12, since $T_{\gamma, d} V$ is locally hyperbolic at 0 . From this fact we get that each noncharacteristic projection for $T_{\gamma, d} V$ at the origin can be used in the definition of local hyperbolicity, in particular, we may use the projection $\pi$ defined above. Consequently, we may assume that $D_{0}$ is so small that $z \in T_{\gamma, d} V \cap 2 D_{0}$ is real whenever $\pi(z)$ is real.

Since $\pi$ is noncharacteristic for $T_{\gamma, d} V$ at $\eta=0$ we get from Lemma 3.10 the existence of $\varepsilon>0$ and $\delta>0$ such that for $D:=B^{2}(0, \varepsilon) \times B^{1}(0, \delta)$ we have $D \subset D_{0}$ and

$$
\pi: T_{\gamma, d} V \cap B^{2}(0,2 \varepsilon) \times B^{1}(0, \delta) \rightarrow B^{2}(0,2 \varepsilon)
$$

is proper. By Lemma 5.8 it also follows that for some $0<\rho_{2} \leq \rho_{1}$

$$
\pi: V \cap \Gamma\left(\gamma, d, D, \rho_{2}\right) \rightarrow \Gamma^{\prime}\left(\pi \circ \gamma, d, B^{2}(0, \varepsilon), \rho_{2}\right)
$$

is proper.

To show that $V$ is $d$-hyperbolic at $\eta=0$, we consider two cases:

Case 1: There exists $\rho>0$ such that $B_{\xi} \cap \mathbb{R}^{3} \cap \Gamma(\gamma, d, D, \rho)=\emptyset$. It is no restriction to assume $\rho \leq \rho_{2}$. Then $V \cap \mathbb{R}^{3}$ is unbranched over

$$
\Gamma^{\prime}:=\Gamma^{\prime}\left(\pi \circ \gamma, d, B^{2}(0, \varepsilon), \rho\right) \cap \mathbb{R}^{2}
$$

with respect to the projection $\pi$. To show that each branch of $V \cap \Gamma(\gamma, d, D, \rho)$ is real over $\Gamma^{\prime}$ choose $\kappa \in T_{\gamma, d} V \cap \mathbb{R}^{3} \cap D$ such that $T_{\gamma, d} V$ is regular at all points $\pi^{-1}(\pi(\kappa)) \cap B^{2}(0,2 \varepsilon) \times B^{1}(0, \delta)$. Then define $\tilde{\kappa}:=\pi(\kappa)$ and

$$
\left.\tilde{\sigma}(t):=\pi \circ \gamma(t)+\tilde{\kappa} t^{d}, t \in\right] 0, \rho[.
$$

Obviously, $\tilde{\sigma}$ is an analytic curve in $\Gamma^{\prime}$. Now fix a branch $V_{0}$ of $V \cap \Gamma(\gamma, d, D, \rho) \cap$ $\pi^{-1}\left(\Gamma^{\prime}\right)$. Since $V \cap \Gamma$ is unbranched over $\Gamma$, we can set $\sigma:=\left(\left.\pi\right|_{V_{0}}\right)^{-1} \circ \tilde{\sigma}$. Since $\{\sigma(t): t \in] 0, \rho[\} \subset \Gamma(\gamma, d, D, \rho)$, we can write

$$
\sigma(t)=\gamma(t)+\hat{\kappa} t^{d}+o\left(t^{d}\right)
$$

for suitable $\hat{\kappa} \in \mathbb{C}^{3}$. It is easy to check that $\hat{\kappa} \in \bar{D} \cap T_{\gamma, d} V$. Since $\pi(\hat{\kappa})-\kappa=o\left(t^{d}\right)$, we have $\pi(\hat{\kappa})=\tilde{\kappa}$ and hence $\hat{\kappa} \in \pi^{-1}(\pi(\tilde{\kappa})) \cap \bar{D} \cap T_{\gamma, d} V$. By the choice of $D$, this implies $\hat{\kappa} \in \mathbb{R}^{3}$. Since our choices also imply that $\hat{\kappa}$ is a regular point of $T_{\gamma, d} V, V$ is $(\gamma, d)$ hyperbolic at $\hat{\kappa} \in T_{\gamma, d} V \cap \mathbb{R}^{3}$. Hence it follows from Lemma 3.19 that $V$ is $(\gamma, d)$ hyperbolic with respect to the given projection $\pi$. Thus there exist an open zero neighborhood $G_{0}$ and $\rho_{0}>0$ such that for each $z \in V \cap \Gamma\left(\gamma, d, \hat{\kappa}+G_{0}, \rho_{0}\right)$ with $\pi(z)$ real, $z$ is already real. Since $\operatorname{tr}(\sigma) \cap B^{3}(0, \alpha)$ is contained in $V_{0} \cap \Gamma\left(\gamma, d, \hat{\kappa}+G_{0}, \rho_{0}\right)$ for $\alpha>0$ sufficiently small, this implies $V_{0} \subset \mathbb{R}^{3}$ since $\left.\pi\right|_{V_{0}}: V_{0} \rightarrow \Gamma^{\prime}$ is unbranched. Since $V_{0}$ was any branch of $V \cap \Gamma(\gamma, d, D, \rho) \cap \pi^{-1}\left(\Gamma^{\prime}\right)$, we proved that $V$ is $(\gamma, d)$ hyperbolic at $\eta=0$.

Case 2: For each $\rho>0$ : $B_{\xi} \cap \mathbb{R}^{3} \cap \Gamma(\gamma, d, D, \rho) \neq \emptyset$. Then we fix $\rho$ and choose a real simple curve $\lambda$ such that $B_{\xi} \cap \mathbb{R}^{3} \cap \Gamma(\gamma, d, D, \rho)$ is the trace of $\lambda$. 
If $\lambda$ is not equivalent to $\gamma$ modulo $d$, then there are a zero neighborhood $G$ and $0<\rho_{1}<\rho$ such that $B_{\xi} \cap \mathbb{R}^{3} \cap \Gamma\left(\gamma, d, G, \rho_{1}\right)=\emptyset$ and we are in Case 1 . Hence we may assume that $\lambda$ is equivalent to $\gamma$ modulo $d$. From this it easily follows that we even may assume $\lambda=\gamma$. Next note that the hypothesis together with the special form of $T_{\gamma, d} V$, described by (5.13), imply that we can find regular points $\kappa, \nu$ in $T_{\gamma, d} V \cap \mathbb{R}^{3} \cap D$ with $\kappa_{2}>0$ and $\nu_{2}<0$ such that all points in $\pi^{-1}(\pi(\kappa)) \cap 2 D$ and in $\pi^{-1}(\pi(\nu)) \cap 2 D$ are regular for $T_{\gamma, d} V$. Using these points we can argue as in Case 1 to show that for

$$
U_{ \pm}:=\left\{x \in \Gamma^{\prime}\left(\pi \circ \gamma, d, B^{2}(0, \varepsilon), \rho\right) \cap \mathbb{R}^{2}: \pm x_{2}>\gamma_{2}\left(x_{1}\right)\right\}
$$

all points of the sets $M_{ \pm}:=V \cap \pi^{-1}\left(U_{ \pm}\right) \cap \Gamma(\gamma, d, D, \rho)$ are real. Since

$$
V \cap \pi^{-1}\left(\Gamma^{\prime}\left(\pi \circ \gamma, d, B^{2}(0, \varepsilon), \rho\right) \cap \mathbb{R}^{2}\right)
$$

is contained in the closure of $M_{+} \cup M_{-}$, it follows that $V$ is $d$-hyperbolic at $\eta=0$.

To also treat points $\xi \in T_{\gamma, d} V \cap \mathbb{R}^{3}$ which are not simple, we will use the following technical lemma which is a refined version of [7], Lemmas 5.9 and 5.10. We let $\mathbb{D}:=B^{1}(0,1)$.

5.10. Lemma. Let $\gamma: t \mapsto\left(t, \gamma_{2}(t), \gamma_{3}(t)\right)$ be a real simple curve with $\left|\gamma_{j}(t)\right|=o(|t|)$ for $j=2,3$, and let $\pi:(x, y, z) \mapsto(x, y)$. Fix $R_{j}, r_{j}>0, j=1,2,3$, and set $G=$ $\prod_{j=1}^{3} B\left(0, r_{j}\right), G^{\prime}=\pi(G), D=\prod_{j=1}^{3} B\left(0, R_{j}\right)$, and $D^{\prime}=\pi(D)$. Let $1 \leq d<\Delta$ and $\rho>0$ with $\rho^{\Delta-1} R_{1}<1 / 2$ be given. Let $V \subset \Gamma(\gamma, d, G, \rho)$ be an analytic surface for which $\pi: V \rightarrow \Gamma^{\prime}\left(\pi \circ \gamma, d, G^{\prime}, \rho\right)$ is proper. Furthermore, assume:

(a) If $z \in V \backslash \Gamma(\gamma, \Delta, 3 D, \rho)$ satisfies $\pi(z) \in \mathbb{R}^{2}$, then $z \in \mathbb{R}^{3}$.

(b) There is no $z \in V$ of the form $z=\gamma(t)+t^{\Delta} w$ with $0<t<\rho, \pi(w) \in 3 D^{\prime}$, and $R_{3} \leq\left|w_{3}\right| \leq 2 R_{3}$.

(c) $V$ satisfies $\operatorname{PL}(V, \Gamma(\gamma, \Delta, 3 D, \rho))$.

Then there are $A, \delta, \rho_{3}>0$ such that, whenever $u \in \operatorname{PSH}(V)$ satisfies

$(\alpha) u(z) \leq|z|^{d}$ for all $z \in V$

and

( $\beta) u(z) \leq 0$ for all $z \in V \cap \mathbb{R}^{3}$,

then $u$ also satisfies

$(\gamma) u(z) \leq A|\operatorname{Im} z|$ for all $z \in V \cap \Gamma\left(\gamma, d, \delta G, \rho_{3}\right)$.

Proof. Since $\left|\left(\gamma_{2}(t), \gamma_{3}(t)\right)\right|=o(|t|)$, there is a constant $C_{1}>0$ such that $|z| \leq$ $C_{1}\left|z_{1}\right|$ whenever $z \in \Gamma(\gamma, d, G, \rho)$, provided $\rho$ is sufficiently small.

Since $\gamma$ is given by a Puiseux series, it can be continued holomorphically to some slit disk $\left.\left.B\left(0, \rho_{1}\right) \backslash\right]-\rho_{1}, 0\right]$. Set

$$
\delta_{3}=\frac{R_{2}}{30 R_{1}} .
$$

Since $\left|\gamma_{2}(t)\right|=o(|t|)$ there is $\rho_{2} \leq \min \left(\rho, \rho_{1}\right)$ such that

$$
\left|\gamma_{2}^{\prime}(t)\right| \leq \delta_{3} \quad \text { for } 0<t<\rho_{2} .
$$


Let $\lambda>0$ be determined by $\lambda^{\Delta}=5 / 4$. Set

$$
\begin{array}{lll}
\epsilon:=\min \left(r_{2}, \frac{\lambda-1}{2 \lambda}\right), & \delta:=\min \left(\frac{3 \epsilon}{4 \lambda^{d} r_{1}}, \frac{3 \epsilon}{4 \lambda^{d}\left(\delta_{3} r_{1}+r_{2}\right)}, \frac{\lambda-1}{\lambda r_{1}}\right), \\
L:=\frac{31 R_{2}}{24 \epsilon}, & \rho_{3}:=\min \left(\frac{2}{3} \rho, \frac{1}{3}\left(\frac{\lambda-1}{6 \lambda R_{1}}\right)^{1 /(\Delta-1)}, \frac{\rho_{2}}{\lambda}, \frac{1}{2^{1 / \Delta}}\right) .
\end{array}
$$

Fix $u \in \operatorname{PSH}(V)$ satisfying $(\alpha)$ and $(\beta)$. As an auxiliary result, we claim that there is $A_{1}>0$, not depending on $u$, such that

$$
\begin{aligned}
& u(z) \leq A_{1}|\operatorname{Im} z|, \quad z \in V \cap \Gamma\left(\gamma, d, \delta G, \rho_{3}\right), \pi(z) \notin \Gamma^{\prime}\left(\pi \circ \gamma, \Delta, 2 D^{\prime}, \rho_{3}\right), \\
& u(z) \leq A_{1}|z|^{\Delta}, \quad z \in V \cap \Gamma\left(\gamma, d, \delta G, \rho_{3}\right), \pi(z) \in \Gamma^{\prime}\left(\pi \circ \gamma, \Delta, 3 D^{\prime}, \rho_{3}\right) .
\end{aligned}
$$

To prove this claim, define

$$
v: \Gamma^{\prime}\left(\pi \circ \gamma, d, G^{\prime}, \rho\right) \rightarrow\left[-\infty, \infty\left[, \quad z^{\prime} \mapsto \max \left\{u(z): z \in V, \pi(z)=z^{\prime}\right\} .\right.\right.
$$

Using the fact that $\pi: V \rightarrow \Gamma^{\prime}\left(\pi \circ \gamma, d, G^{\prime}, \rho\right)$ is proper together with the theorem about removable singularities of plurisubharmonic functions (Hörmander, [17, 4.4), it is easy to see that $v$ is plurisubharmonic. By $(\alpha)$ and the choice of $C_{1}$ we have $v\left(z^{\prime}\right) \leq\left(C_{1}\left|z_{1}\right|\right)^{d}$.

For now let $0<t_{0}<\rho_{3}$ be arbitrary and define

$$
\psi: \mathbb{D} \times \mathbb{D} \rightarrow \mathbb{C}^{2}, \quad(a, b) \mapsto\left(t_{0}+\epsilon t_{0}^{d} a, \gamma_{2}\left(t_{0}+\epsilon t_{0}^{d} a\right)+\epsilon t_{0}^{d} b\right) .
$$

We have $\epsilon<1 / 2$, hence $0<t_{0}+\epsilon t_{0}^{d} a<(3 / 2) \rho_{3}<\rho$ and $\psi(a, b) \in \Gamma^{\prime}\left(\pi \circ \gamma, d, G^{\prime}, \rho\right)$. In particular, we have $v \circ \psi(a, b) \leq\left(C_{1}\left(t_{0}+\epsilon t_{0}^{d}\right)\right)^{d} \leq C_{1}^{d} 2^{d} t_{0}^{d}$. Now fix $a \in \mathbb{D}$ and $b \in]-1,1[\backslash]-L t_{0}^{\Delta-d}, L t_{0}^{\Delta-d}[$. We claim

$$
\psi(a, b) \notin \Gamma^{\prime}\left(\pi \circ \gamma, \Delta, D^{\prime}, \rho\right) .
$$

To prove this by contradiction, let us assume the existence of $t \in] 0, \rho\left[\right.$ and $w \in D^{\prime}$ with

$$
\psi(a, b)=\gamma(t)+t^{\Delta} w
$$

Comparison of the first coordinate yields

$$
t_{0}+\epsilon t_{0}^{d} a=t+t^{\Delta} w_{1} .
$$

Since $t_{0}<\rho_{3}$, this implies $\left|t\left(1+t^{\Delta-1} w_{1}\right)\right| \leq \rho_{3}+\epsilon \rho_{3}^{d}<3 \rho_{3} / 2$, while $\left|1+t^{\Delta-1} w_{1}\right| \geq$ $1-\rho^{\Delta-1} R_{1}>1 / 2$. Hence $t \leq 3 \rho_{3}$ and $t^{\Delta-1}\left|w_{1}\right| \leq\left(3 \rho_{3}\right)^{\Delta-1} R_{1} \leq(\lambda-1) /(2 \lambda)$. Together with $\epsilon|a|<(\lambda-1) /(2 \lambda)$ this leads to

$$
\begin{aligned}
\left|t_{0}-t\right| & =\left|t^{\Delta} w_{1}-\epsilon t_{0}^{d} a\right|=\left|\left(t-t_{0}\right) t^{\Delta-1} w_{1}+t_{0}\left(t^{\Delta-1} w_{1}-\epsilon t_{0}^{d-1} a\right)\right| \\
& \leq\left|t-t_{0}\right| \frac{\lambda-1}{2 \lambda}+t_{0} \frac{\lambda-1}{\lambda} .
\end{aligned}
$$

So far, we have shown $(1 / \lambda)\left|t_{0}-t\right| \leq(\lambda-1) t_{0} / \lambda$ and thus

$$
t<\lambda t_{0}<\rho_{2} \text {. }
$$

Comparison of the second coordinate of (15.18) yields $\gamma_{2}\left(t_{0}+\epsilon t_{0}^{d} a\right)+\epsilon t_{0}^{d} b=\gamma_{2}(t)+$ $t^{\Delta} w_{2}$. We isolate $w_{2}$ :

$$
w_{2}=\epsilon t_{0}^{d} t^{-\Delta} b+\left(\gamma_{2}\left(t_{0}+\epsilon t_{0}^{d} a\right)-\gamma_{2}(t)\right) t^{-\Delta} .
$$


This equation is exploited using (5.19) and $|b|>L t_{0}^{\Delta-d}$ :

$$
\begin{aligned}
&\left|w_{2}\right| \geq \epsilon t_{0}^{d} t^{-\Delta}|b|-\left|t_{0}+\epsilon t_{0}^{d} a-t\right| \delta_{3} t^{-\Delta} \geq \epsilon t_{0}^{d-\Delta}|b|\left(\frac{t_{0}}{t}\right)^{\Delta}-\left|t^{\Delta} w_{1}\right| \delta_{3} t^{-\Delta} \\
& \geq \frac{4}{5} \frac{31}{24} R_{2}-\delta_{3} R_{1}=R_{2} .
\end{aligned}
$$

As this contradicts $w \in D^{\prime}$, claim (5.17) is shown. Now set

$$
\phi(a, b):=\frac{1}{C_{1}^{d} 2^{d} t_{0}^{d}} v \circ \psi(a, b) .
$$

Then

$$
\begin{array}{ll}
\phi(a, b) \leq 1 & \text { for }(a, b) \in \mathbb{D} \times \mathbb{D}, \\
\phi(a, b) \leq 0 & \text { for }(a, b) \in]-1,1\left[\times(]-1,1[\backslash]-L t_{0}^{\Delta-d}, L t_{0}^{\Delta-d}[) .\right.
\end{array}
$$

By [7, Lemma 5.8, there is $C_{3}>0$, not depending on $u$, such that

$$
\phi(a, b) \leq C_{3}\left(|\operatorname{Im} a|+\left|\operatorname{Im} \sqrt{b^{2}-L^{2} t_{0}^{2(\Delta-d)}}\right|\right) \quad \text { for } a, b \in B(0,3 / 4) .
$$

If $|b|>(3 / 2) L t_{0}^{\Delta-d}$, then [7, Lemma 5.7, implies $\left|\operatorname{Im} \sqrt{b^{2}-L^{2} t_{0}^{2(\Delta-d)}}\right| \leq$ $3|\operatorname{Im} b| / \sqrt{5}$. On the other hand, if $|b| \leq 3 L t_{0}^{\Delta-d}$, then $\left|\operatorname{Im} \sqrt{b^{2}-L^{2} t_{0}^{2(\Delta-d)}}\right| \leq$ $4 L t_{0}^{\Delta-d}$. This term can be estimated by a multiple of $|\operatorname{Im} a|$ provided $|\operatorname{Im} a|$ is large enough. Hence there is $C_{4}$, not depending on $u$, such that for $a, b \in B(0,3 / 4)$,

$$
\phi(a, b) \leq \begin{cases}C_{4}|\operatorname{Im}(a, b)| & \text { if }|b|>\frac{3}{2} L t_{0}^{\Delta-d} \text { or }|\operatorname{Im} a|>\frac{R_{1}}{\epsilon} t_{0}^{\Delta-d}, \\ C_{4} t_{0}^{\Delta-d} & \text { if }|b| \leq 3 L t_{0}^{\Delta-d} \text { and }|\operatorname{Im} a| \leq 4 \frac{R_{1}}{\epsilon} t_{0}^{\Delta-d} .\end{cases}
$$

To prove (5.15) and (5.16), fix $z \in \Gamma\left(\gamma, d, \delta G, \rho_{3}\right)$. Then there are $w \in \delta G$ and $0<t<\rho_{3}$ with

$$
z=\gamma(t)+t^{d} w
$$

Of course, $t$ and $w$ are not unique. We start with some estimates that hold for all choices of $t$ and $w$. Finally, the proof of (5.15) and (5.16) will be broken into several cases with different additional assumptions concerning $t$ and $w$.

$$
t_{0}:=\operatorname{Re} z_{1}, \quad a:=\frac{i}{\epsilon t_{0}^{d}} \operatorname{Im} z_{1}, \quad b:=\frac{1}{\epsilon t_{0}^{d}}\left(z_{2}-\gamma_{2}\left(z_{1}\right)\right) .
$$

Note that $\left|\operatorname{Im} w_{1}\right| \leq \delta r_{1} \leq 1-1 / \lambda$. Hence comparison of the real parts of the first coordinate in (5.21) gives $t=t_{0}-t^{d} \operatorname{Re} w_{1} \leq t_{0}+t(1-1 / \lambda)$ and thus

$$
t_{0} \leq|z| \text { and } t \leq \lambda t_{0} .
$$

Equation (5.21) also implies

$$
|a|=\frac{1}{\epsilon t_{0}^{d}}\left|\operatorname{Im} z_{1}\right|=\frac{1}{\epsilon}\left(\frac{t}{t_{0}}\right)^{d}\left|\operatorname{Im} w_{1}\right|<\frac{\delta}{\epsilon} \lambda^{d} r_{1} \leq \frac{3}{4} .
$$


To derive an estimate for $|b|$ we use (5.14)

$$
\begin{aligned}
|b|=\frac{1}{\epsilon t_{0}^{d}} \mid \gamma_{2}(t)-\gamma_{2}\left(z_{1}\right) & +t^{d} w_{2} \mid \leq \frac{1}{\epsilon t_{0}^{d}}\left(\delta_{3}\left|t-z_{1}\right|+t^{d}\left|w_{2}\right|\right) \\
& \leq \frac{1}{\epsilon}\left(\frac{t}{t_{0}}\right)^{d}\left(\delta_{3}\left|w_{1}\right|+\left|w_{2}\right|\right) \leq \frac{\lambda^{d}}{\epsilon}\left(\delta_{3}\left|w_{1}\right|+\left|w_{2}\right|\right) .
\end{aligned}
$$

The definition of $\delta$ is such that (5.23) implies $|b|<3 / 4$ and thus $a, b \in B(0,3 / 4)$.

Then $\psi(a, b)=\pi(z)$ is obvious. We will also need an estimate for $|\operatorname{Im} b|$ later on:

$$
\begin{aligned}
& \quad|\operatorname{Im} b|=\frac{1}{\epsilon t_{0}^{d}}\left|\operatorname{Im} z_{2}-\operatorname{Im} \gamma_{2}\left(z_{1}\right)\right| \leq \frac{1}{\epsilon t_{0}^{d}}\left(\left|\operatorname{Im} z_{2}\right|+\left|\operatorname{Im}\left(\gamma_{2}\left(z_{1}\right)-\gamma_{2}\left(\operatorname{Re} z_{1}\right)\right)\right|\right) \\
& \leq \frac{1}{\epsilon t_{0}^{d}}\left(\left|\operatorname{Im} z_{2}\right|+\left|\gamma_{2}\left(z_{1}\right)-\gamma_{2}\left(\operatorname{Re} z_{1}\right)\right|\right) \leq \frac{1}{\epsilon t_{0}^{d}}\left(\left|\operatorname{Im} z_{2}\right|+\left|\operatorname{Im} z_{1}\right| \delta_{3}\right) \leq \frac{2}{\epsilon t_{0}^{d}}|\operatorname{Im} z| .
\end{aligned}
$$

We have to distinguish three cases:

Case $\pi(z) \in \Gamma^{\prime}\left(\pi \circ \gamma, \Delta, 3 D^{\prime}, \rho_{3}\right)$ : Then it is possible to pick $t$ and $w$ in (5.21) such that $\pi(w) \in 3 t^{\Delta-d} D^{\prime}$. Hence

$$
|\operatorname{Im} a|=\frac{1}{\epsilon t_{0}^{d}}\left|\operatorname{Im} z_{1}\right|=\frac{1}{\epsilon}\left(\frac{t}{t_{0}}\right)^{d}\left|\operatorname{Im} w_{1}\right| \leq \frac{3}{\epsilon}\left(\frac{t}{t_{0}}\right)^{\Delta} t_{0}^{\Delta-d} R_{1} \leq 4 \frac{R_{1}}{\epsilon} t_{0}^{\Delta-d} .
$$

Concerning $|b|$, we get from (5.23)

$$
\begin{aligned}
|b| & \leq \frac{3}{\epsilon} \lambda^{d}\left(\delta_{3} R_{1}+R_{2}\right) t^{\Delta-d} \leq \frac{3}{\epsilon} \lambda^{\Delta}\left(\delta_{3} R_{1}+R_{2}\right) t_{0}^{\Delta-d} \\
& \leq \frac{3}{\epsilon} \frac{5}{4} \frac{31}{30} R_{2} t_{0}^{\Delta-d}=3 L t_{0}^{\Delta-d} .
\end{aligned}
$$

By (5.20) this implies $\phi(a, b) \leq C_{4} t_{0}^{\Delta-d}$ and hence (5.16) follows from

$$
u(z) \leq 2^{d} C_{4} C_{1}^{d} t_{0}^{\Delta} \leq 2^{d+1} C_{4} C_{1}^{d}|z|^{\Delta} .
$$

Case $\left|\operatorname{Im} z_{1}\right|>R_{1} t_{0}^{\Delta}$ : Then

$$
|\operatorname{Im} a|=\frac{1}{\epsilon t_{0}^{d}}\left|\operatorname{Im} z_{1}\right| \geq \frac{R_{1}}{\epsilon} t_{0}^{\Delta-d} .
$$

Hence we are in the first case of (5.20), and claim (5.15) follows using (5.24):

$$
u(z) \leq 2^{d} C_{4} C_{1}^{d} t_{0}^{d}|\operatorname{Im}(a, b)| \leq \frac{2^{d+2} C_{4} C_{1}^{d}}{\epsilon}|\operatorname{Im} z| .
$$

Case $\left|\operatorname{Im} z_{1}\right| \leq R_{1} t_{0}^{\Delta}$ and $\pi(z) \notin \Gamma^{\prime}\left(\pi \circ \gamma, \Delta, 2 D^{\prime}, \rho_{3}\right)$ : Then

$$
z=\gamma\left(t_{0}\right)+t_{0}^{\Delta} x \text { for } \quad x=\frac{z-\gamma\left(t_{0}\right)}{t_{0}^{\Delta}} .
$$

The hypothesis of this case implies $\pi(x) \notin 2 D^{\prime}$. Investigate its first coordinate:

$$
\left|x_{1}\right|=t_{0}^{-\Delta}\left|z_{1}-\operatorname{Re} z_{1}\right|=t_{0}^{-\Delta}\left|\operatorname{Im} z_{1}\right| \leq R_{1}<2 R_{1} .
$$

Hence the second coordinate must satisfy $\left|x_{2}\right| \geq 2 R_{2}$. Because

$$
b=\frac{1}{\epsilon} t_{0}^{\Delta-d} x_{2}+\frac{1}{\epsilon t_{0}^{d}}\left(\gamma_{2}\left(\operatorname{Re} z_{1}\right)-\gamma_{2}\left(z_{1}\right)\right),
$$

the estimate for $\left|x_{2}\right|$, the assumption of this case and (5.14) imply

$$
|b| \geq \frac{2}{\epsilon} t_{0}^{\Delta-d} R_{2}-\frac{1}{\epsilon t_{0}^{d}}\left|\operatorname{Im} z_{1}\right| \delta_{3} \geq \frac{t_{0}^{\Delta-d}}{\epsilon}\left(2 R_{2}-\delta_{3} R_{1}\right) \geq \frac{31}{16} \frac{R_{2}}{\epsilon} t_{0}^{\Delta-d}=\frac{3}{2} L t_{0}^{\Delta-d} .
$$


Thus (5.20) and (5.24) imply

$$
u(z) \leq 2^{d} C_{1}^{d} C_{4} t_{0}^{d}|\operatorname{Im}(a, b)| \leq \frac{2^{d+2} C_{1}^{d} C_{4}}{\epsilon}|\operatorname{Im} z| .
$$

This proves (5.15) also in the remaining case.

Thus the proof of the intermediate claim is complete. To complete the proof of the lemma, note first that by (5.15) the claim is shown for $z \in V \cap \Gamma\left(\gamma, d, \delta G, \rho_{3}\right)$ with $\pi(z) \notin \Gamma^{\prime}\left(\pi \circ \gamma, \Delta, 2 D^{\prime}, \rho_{3}\right)$. On the other hand, if $\pi(z) \in \Gamma^{\prime}\left(\pi \circ \gamma, \Delta, 2 D^{\prime}, \rho_{3}\right)$, then there are two cases. First, for $z \in V \cap \Gamma\left(\gamma, \Delta, 2 D, \rho_{4}\right), \rho_{4}>0$, sufficiently small, the claim follows from hypothesis (c) since (5.16) gives estimate $(\alpha)$ of $\operatorname{PL}(V, \Gamma(\gamma, \Delta, 3 D, \rho))$. For the remaining case, consider

$$
\begin{aligned}
w: \Gamma^{\prime}\left(\pi \circ \gamma, \Delta, 3 D^{\prime}, \rho_{3}\right) \rightarrow & {[-\infty, \infty[} \\
z^{\prime} & \mapsto \max \left\{u(z): z \in V \cap \Gamma\left(\gamma, d, G, \rho_{3}\right) \backslash \Gamma\left(\gamma, \Delta, 3 D, \rho_{3}\right), \pi(z)=z^{\prime}\right\} .
\end{aligned}
$$

Then $w$ is plurisubharmonic by (b) and the argument concerning removal of singularities in [17, 4.4. We have $w\left(z^{\prime}\right) \leq A_{1}|z|^{\Delta}$ by (5.16) and $w\left(z^{\prime}\right) \leq 0$ for all real $z^{\prime}$ by (a) and estimate $(\beta)$ of $\operatorname{PL}\left(V, \Gamma\left(\gamma, d, \delta G, \rho_{3}\right)\right)$. Hence the arguments that were used for the proof of Lemma 5.8 also apply here, and we get

$$
w\left(z^{\prime}\right) \leq A\left|\operatorname{Im} z^{\prime}\right| \quad \text { for } z^{\prime} \in \Gamma^{\prime}\left(\pi \circ \gamma, \Delta, 2 D^{\prime}, \rho_{4}\right)
$$

for suitable $A, \rho_{4}>0$. This completes the proof.

Using Lemma 5.10 we are now able to prove:

5.11. Lemma. Let $V$ be an analytic surface in $\mathbb{C}^{3}$ which contains the origin, let $\gamma$ : ] $0, \alpha\left[\rightarrow \mathbb{R}^{3}\right.$ be a real simple curve, $d \geq 1$, and assume $0 \in T_{\gamma, d} V$. Let $\Delta:=\Delta^{A}(\gamma, d)$ for $A:=T_{0}\left(T_{\gamma, d} V\right) \cup T_{0} V$ and assume that the following conditions are satisfied:

(a) $0 \in T_{\gamma, d} V$ is not a simple point of $T_{\gamma, d} V$.

(b) $T_{\gamma, d} V$ is locally hyperbolic at each real point.

(c) For each $\eta \in T_{\gamma, d} V \cap \mathbb{R}^{3}$ which is regular, $V$ is $(\gamma, d)$-hyperbolic at $\eta$.

(d) For each $\zeta \in T_{\gamma, \Delta} V \cap \mathbb{R}^{3}$ there exist a zero neighborhood $D_{\zeta}$ and $\rho_{\zeta}>0$ such that $V$ satisfies $\operatorname{PL}\left(V, \Gamma\left(\gamma, \Delta, \zeta+D_{\zeta}, \rho_{\zeta}\right)\right)$.

Then there exist an open zero neighborhood $G$ and $\rho>0$ such that $V$ satisfies $\operatorname{PL}(V, \Gamma(\gamma, d, G, \rho))$.

Proof. By (a) and Lemma 4.8 we get $\Delta<\infty$ and $T_{\gamma, \Delta} V \neq \emptyset$. Hence we can choose $\xi \in \mathbb{R}^{3},|\xi|=1$ satisfying

$$
\xi \notin T_{0}\left(T_{\gamma, d} V\right),
$$

$\xi$ is not characteristic for the algebraic variety $T_{\gamma, \Delta} V$.

Moreover, (a) implies the existence of $k \in \mathbb{N}, k \geq 2$, such that there are $k$ different branches $S_{1}, \ldots, S_{k}$ of $B_{\xi} \cap \mathbb{R}^{3}$ which are equivalent modulo $d$ to $\gamma$. Denote by $\xi_{1} \in \mathbb{R}^{3},\left|\xi_{1}\right|=1$, their common tangent at the origin and choose $\xi_{2} \in \mathbb{R}^{3},\left|\xi_{2}\right|=1$, so that $\left(\xi_{1}, \xi_{2}, \xi\right)$ are linearly independent. Modulo a real linear change of variables we may assume that $\left(\xi_{1}, \xi_{2}, \xi\right)=\left(e_{1}, e_{2}, e_{3}\right)$ are the standard basis vectors of $\mathbb{R}^{3}$.

Since $e_{1}$ is tangent to $\gamma(] 0, \alpha[)$ in the origin, Lemma 2.5 implies that we may assume that $\gamma$ is in standard parametrization with respect to the basis $\left(e_{1}, e_{2}, e_{3}\right)$; consequently we have

$$
\gamma(t)=\left(t, \gamma_{2}(t), \gamma_{3}(t)\right),\left|\gamma_{j}(t)\right|=o(|t|), j=2,3 .
$$


Then note that (5.25) and (5.26) imply

$$
\begin{gathered}
\pi: \mathbb{C}^{3} \rightarrow \mathbb{C}^{3}, \pi\left(z_{1}, z_{2}, z_{3}\right):=\left(z_{1}, z_{2}, 0\right) \text { is noncharacteristic for } T_{\gamma, d} V, \\
\text { there exists } C \geq 1 \text { such that }|z| \leq C(1+|\pi(z)|), \text { for all } z \in T_{\gamma, \Delta} V .
\end{gathered}
$$

Since $T_{\gamma, d} V$ is locally hyperbolic at zero by (b), it follows from Braun [5], Corollary 12 , and (5.25) that $T_{\gamma, d} V$ is locally hyperbolic with respect to the projection $\pi$. Hence we can choose an open zero neighborhood $G$ in $\mathbb{C}^{3}$ such that the following holds:

$$
\text { If } z \in T_{\gamma, d} V \cap G \text { and } \pi(z) \text { is real, then } z \text { is real. }
$$

By Lemma 4.10, the set $\mathcal{M}_{\gamma, d}$ of all nonsimple points of $T_{\gamma, d} V$ is a finite union of real lines, parallel to $e_{1}$ when $d>1$ and $\mathcal{M}_{\gamma, 1} \backslash\left\{-e_{1}\right\}$ is a finite union of open real rays for each of which $-e_{1}$ is an adherent point. Letting $\widetilde{\mathcal{M}}_{\gamma, d}:=\mathcal{M}_{\gamma, d}$ if $d>1$ and $\widetilde{\mathcal{M}}_{\gamma, 1}:=\mathcal{M}_{\gamma, 1} \backslash\left\{-e_{1}\right\}$ if $d=1$, we can choose $G$ so small that the following holds:

$$
G \cap \widetilde{\mathcal{M}}_{\gamma, d} \text { is connected. }
$$

Furthermore we may choose $\rho_{1}>0$ and $G$ so that

$$
\text { Each branch } T \text { of } B_{\xi} \cap \mathbb{R}^{3} \text { which satisfies } T \cap \Gamma\left(\gamma, d, G, \rho_{1}\right) \neq \emptyset \text { is }
$$
equivalent to $\gamma$ modulo $d$.

Note that (5.30), (5.31), and (5.32) remain true if we replace $G$ by any smaller zero neighborhood and $\rho_{1}$ by any smaller positive number. Hence we can apply Lemma 5.8 to get $\varepsilon>0, \delta>0$, and $\rho_{1}>0$ such that for $G:=B(0, \varepsilon)^{2} \times B(0, \delta)$ we have (5.30), (5.31), and (5.32) as well as

$$
\pi: V \cap \Gamma\left(\gamma, d, G, \rho_{1}\right) \rightarrow \Gamma^{\prime}\left(\pi \circ \gamma, d, G^{\prime}, \rho_{1}\right)
$$

is proper.

Next note that by the definition of $\Delta$ we can choose $R_{1}>0$ and $0<\rho_{2} \leq \rho_{1}$ such that for $D_{1}:=B\left(0, R_{1}\right)^{2} \times B\left(0, C(1+\sqrt{2}) R_{1}\right)$ we have $\Gamma\left(\gamma, \Delta, D_{1}, \rho_{2}\right) \subset$ $\Gamma\left(\gamma, d, G, \rho_{2}\right)$ and

Each branch $T$ of $B_{\xi} \cap \mathbb{R}^{3}$ which is equivalent to $\gamma$ modulo $d$ is contained in $\Gamma\left(\gamma, \Delta, D_{1}, \rho_{2}\right)$.

Now note that by the hypotheses (b) and (c) we can use the same arguments as in the proof of Case 1 in Lemma 5.9 to prove the following (shrinking $\rho_{2}$ if necessary):

For each $z \in V \cap \Gamma\left(\gamma, d, G, \rho_{2}\right)$ for which $\pi(z)$ is real and $\pi(z) \notin$ $\Gamma^{\prime}\left(\pi \circ \gamma, \Delta, D_{1}^{\prime}, \rho_{2}+\varepsilon\right), z$ is real.

Next we claim:

For $R>0$ let $S:=2 C(2 R+5)+2$, where $C$ is the constant in (5.29). Then for each $L>S$ there is $r>0$ such that $0<t<r$, $\zeta \in B(0, R)^{2} \times B(0, L)$, and $\gamma(t)+t^{\Delta} \zeta \in V$ imply $\left|\zeta_{3}\right|<S$.

To prove (5.35) fix $R>0$ and $L>S$, let $\Omega:=B(0, R)^{2} \times B(0, L)$, and choose $\eta>0$ so small that $\eta 2^{\Delta}<1,\left(1-\eta 2^{\Delta}\right)^{-\Delta} \leq 2$, and $\left(1+\eta 2^{\Delta}\right)^{\Delta} \leq 2$. Since $\gamma$ is in standard parametrization, $\gamma$ can be extended analytically to $B(0, \alpha) \backslash]-\infty, 0]$ and we can choose $0<r_{0}<\alpha$ such that

$$
\left.\left.|\gamma(t)|+t^{\Delta}(L+2 R) \leq 2 t, t \in\right] 0, r_{0}\left[, \quad\left|\gamma_{j}^{\prime}(s)\right| \leq \frac{1}{2^{\Delta}}, s \in B\left(0, r_{0}\right) \backslash\right]-\infty, 0\right], j=2,3 .
$$


Moreover, it follows from [9], Proposition 5.4, that there is $0<r \leq r_{0} /\left(1+\eta 2^{\Delta}\right)$ such that for each $z \in V \cap \Gamma(\gamma, \Delta, \Omega, r)$ there exist $\tau \in B(0, \alpha) \backslash]-\infty, 0]$ and $v \in T_{\gamma, \Delta} V$ satisfying $v_{1}=0$ such that for $w:=\gamma(\tau)+\tau^{\Delta} v$ we have

$$
|z-w| \leq \eta|z|^{\Delta} \text {. }
$$

Next fix $z \in V \cap \Gamma(\gamma, \Delta, \Omega, r)$ and choose $0<t<r$ and $\zeta \in \Omega$ such that $z=$ $\gamma(t)+t^{\Delta} \zeta$. Let $\tau, v$, and $w$ be as above so that (5.37) holds. Then $\zeta \in \Omega$ and (5.36) imply

$$
|z| \leq|\gamma(t)|+t^{\Delta}|\zeta| \leq|\gamma(t)|+t^{\Delta}(L+2 R) \leq 2 t .
$$

From this and (5.37) we get

$$
|\tau-t|=\left|w_{1}-z_{1}\right| \leq|w-z| \leq \eta|z|^{\Delta} \leq \eta(2 t)^{\Delta} .
$$

By the estimate on $r$, this implies

$$
|\tau| \leq|\tau-t|+t \leq \eta(2 t)^{\Delta}+t \leq t\left(1+\eta 2^{\Delta}\right)<r\left(1+\eta 2^{\Delta}\right) \leq r_{0} .
$$

Hence we can apply (5.36) together with $t>0$ to obtain

$$
\left|\gamma_{j}(\tau)-\gamma_{j}(t)\right| \leq \frac{1}{2^{\Delta}} \eta(2 t)^{\Delta}=\eta t^{\Delta}, \quad j=2,3 .
$$

Also because of $t \leq 1$ we get

$$
t\left(1-2^{\Delta} \eta\right) \leq t-|t-\tau| \leq|\tau| \leq t+|t-\tau| \leq t\left(1+2^{\Delta} \eta\right) .
$$

By the choice of $\eta$ this gives

$$
\left|\frac{t}{\tau}\right|^{\Delta} \leq\left(\frac{1}{1-2^{\Delta} \eta}\right)^{\Delta} \leq 2 \text { and }\left|\frac{\tau}{t}\right|^{\Delta} \leq\left(1+2^{\Delta} \eta\right)^{\Delta} \leq 2 .
$$

Using the first one of these estimates, (5.38), and $\left|\zeta_{2}\right|<R$ we now obtain

$$
\begin{aligned}
\left|v_{2}\right| & =\frac{1}{|\tau|^{\Delta}}\left|w_{2}-\gamma_{2}(\tau)\right| \leq \frac{1}{|\tau|^{\Delta}}\left(\left|w_{2}-z_{2}\right|+\left|z_{2}-\gamma_{2}(t)\right|+\left|\gamma_{2}(\tau)-\gamma_{2}(t)\right|\right) \\
& \leq\left(\frac{t}{|\tau|}\right)^{\Delta}\left(2^{\Delta} \eta+R+\eta\right) \leq 2(R+2)=2 R+4 .
\end{aligned}
$$

Since $v=\left(0, v_{2}, v_{3}\right)$, we get from this and (5.29)

$$
\left|v_{3}\right| \leq|v| \leq C(1+|\pi(v)|) \leq C(1+2 R+4)=C(2 R+5) .
$$

Now note that from this and (5.38) we get

$$
\begin{aligned}
\left|\zeta_{3}\right| & =\frac{1}{t^{\Delta}}\left|z_{3}-\gamma_{3}(t)\right| \leq \frac{1}{t^{\Delta}}\left(\left|z_{3}-w_{3}\right|+\left|w_{3}-\gamma_{3}(\tau)\right|+\left|\gamma_{3}(\tau)-\gamma_{3}(t)\right|\right) \\
& \leq \eta 2^{\Delta}+\left|\frac{\tau}{t}\right|^{\Delta}\left|v_{3}\right|+\eta<2 C(2 R+5)+2=S,
\end{aligned}
$$

which proves (5.35).

For $R_{1}$ as above let $R_{2}:=3 R_{1}, S_{2}:=2 C\left(2 R_{2}+5\right)+2, L_{2}:=S_{2}+2, D_{2}:=$ $B\left(0, R_{2}\right)^{2} \times B\left(0, L_{2}\right)$ and choose $0<\rho_{3}<\rho_{2}$ so that (5.35) holds for these choices of $R, L$, and $\rho_{3}=r$. Shrinking $\rho_{3}$ if necessary, we may assume that for all $\left.t \in\right] 0, \rho_{3}[$ we have

$$
\pi \circ \gamma(t)+\left[-t^{\Delta} R_{1}, t^{\Delta} R_{1}\right] \times\left\{2 R_{1}\right\} \subset \mathbb{R}^{2} \backslash \Gamma^{\prime}\left(\pi \circ \gamma, \Delta, D_{1}^{\prime}, \rho_{3}\right) .
$$

Then we claim that the following assertion holds:

(5.40) If $z \in\left(V \backslash \Gamma\left(\gamma, \Delta, D_{2}, \rho_{3}\right)\right) \cap \Gamma\left(\gamma, d, G, \rho_{3}\right)$ and $\pi(z)$ is real, then $z$ is real. 
To prove (5.40), fix $z_{0}$ as in (5.40). If $\pi\left(z_{0}\right) \notin \Gamma^{\prime}\left(\pi \circ \gamma, \Delta, D_{1}^{\prime}, \rho_{3}\right)$, then $z_{0}$ is real by (5.34). If $\pi\left(z_{0}\right) \in \Gamma^{\prime}\left(\pi \circ \gamma, \Delta, D_{1}^{\prime}, \rho_{3}\right)$, then

$$
\pi\left(z_{0}\right)=\pi \circ \gamma\left(t_{0}\right)+t_{0}^{\Delta}\left(\xi_{1}, \xi_{2}\right),
$$

for some $0<t_{0}<\rho_{3}$ and $\left(\xi_{1}, \xi_{2}\right) \in B\left(0, R_{1}\right)^{2} \cap \mathbb{R}^{2}$. Next define

$$
\tilde{\sigma}:]-R_{2}, R_{2}\left[\rightarrow \mathbb{R}^{2}, \quad \tilde{\sigma}(s):=\pi \circ \gamma\left(t_{0}\right)+t_{0}^{\Delta}\left(\xi_{1}, s\right) .\right.
$$

Since $\pi: V \cap \Gamma\left(\gamma, d, G, \rho_{3}\right) \rightarrow \Gamma^{\prime}\left(\pi \circ \gamma, d, G, \rho_{3}\right)$ is a branched covering map, we can choose a continuous map

$$
\sigma:]-R_{2}, R_{2}\left[\rightarrow V \cap \Gamma\left(\gamma, d, G, \rho_{3}\right) \text { satisfying } \pi \circ \sigma=\tilde{\sigma} \text { and } z_{0}=\sigma\left(\xi_{2}\right) .\right.
$$

By (5.39) we have $\tilde{\sigma}\left(2 R_{1}\right) \in \mathbb{R}^{2} \backslash \Gamma^{\prime}\left(\pi \circ \gamma, \Delta, D_{1}^{\prime}, \rho_{3}\right)$. Hence (5.34) implies that $\sigma\left(2 R_{1}\right)$ is a real regular point of $V$.

Next note that (5.35) and our choices imply

$$
\begin{aligned}
M: & =\{s \in]-R_{2}, R_{2}\left[: \frac{1}{t_{0}^{\Delta}}\left|\sigma_{3}(s)-\gamma_{3}\left(t_{0}\right)\right|<L_{2}\right\} \\
& =\{s \in]-R_{2}, R_{2}\left[: \frac{1}{t_{0}^{\Delta}}\left|\sigma_{3}(s)-\gamma_{3}\left(t_{0}\right)\right|<S_{2}\right\} .
\end{aligned}
$$

Since ] $-R_{2}, R_{2}[$ is connected, $M$ is either empty or $M=]-R_{2}, R_{2}[$. Since

$$
\frac{1}{t_{0}^{\Delta}}\left|\sigma_{3}\left(\xi_{2}\right)-\gamma_{3}\left(t_{0}\right)\right|=\frac{1}{t_{0}^{\Delta}}\left|z_{0,3}-\gamma_{3}\left(t_{0}\right)\right| \geq L_{2}
$$

we must have $M=\emptyset$ and hence

$$
\left.\frac{1}{t_{0}^{\Delta}}\left|\sigma_{3}(s)-\gamma_{3}\left(t_{0}\right)\right| \geq L_{2} \text { for all } s \in\right]-R_{2}, R_{2}[.
$$

This implies that the lifting $\sigma$ cannot pass through any real branch point of $\pi$ on $V \cap \Gamma\left(\gamma, d, G, \rho_{3}\right)$, since all of these lie in $\Gamma\left(\gamma, \Delta, D, \rho_{3}\right)$. Consequently, the lifted curve $s \mapsto \sigma\left(t_{0}+t_{0}^{\Delta} \xi_{1}, \gamma_{2}\left(t_{0}\right)+t_{0}^{\Delta} s, \sigma_{3}(s)\right)$ is unique and real analytic as a function of $s \in]-R_{2}, R_{2}$ [. However, $\sigma(s)$ must be real if $2 R_{1}<s<R_{2}$, so in fact it must be real for each $s \in]-R_{2}, R_{2}$ [. In particular, $z_{0}=\sigma\left(\xi_{2}\right)$ is real, which completes the proof of (5.40).

Now note that by (5.27), (5.32), (5.35), (5.40), and the hypothesis (d) in connection with Lemma 5.6, the hypotheses of Lemma 5.10 are fulfilled for $D:=$ $\frac{1}{3} D_{2}$ and suitable $0<\rho \leq \rho_{3}$. To derive from Lemma 5.10 that $V$ satisfies $\mathrm{PL}(V, \Gamma(\gamma, d, G, \rho))$ let $A, \delta_{0}$, and $\rho_{4}$ be the constants which exist by that lemma. Then fix $u \in \operatorname{PSH}(V \cap \Gamma(\gamma, d, G, \rho))$ satisfying

$$
u(z) \leq|z|^{d}, z \in V \cap \Gamma(\gamma, d, G, \rho) \text { and } u(z)=0 \text { for } z \in V \cap \mathbb{R}^{3} \cap \Gamma(\gamma, d, G, \rho) .
$$

By Lemma 5.10, this implies

$$
u(z) \leq A|\operatorname{Im} z|, z \in V \cap \Gamma\left(\gamma, d, \delta_{0} G, \rho_{4}\right) .
$$

To conclude from (5.41) that $V$ satisfies $\operatorname{PL}(V, \Gamma(\gamma, d, G, \rho))$ assume first that $d=1$ and fix a compact subset $K$ of $G$, w.l.o.g. $K=B^{2}\left(0, \varepsilon_{1}\right) \times B^{1}\left(0, \delta_{1}\right)$ for suitable $0<\varepsilon_{1}<\varepsilon$ and $0<\delta_{1}<\delta$. From (5.31) and Lemma 4.10(a) it follows that each point $\eta \in K \cap \mathbb{R}^{3} \backslash \mathbb{R} \times\{0\} \times\{0\}$ is a simple point of $T_{\gamma, 1} V$. Therefore, it follows from condition (b), Lemma 5.9, and Lemma 5.7, that we can find a zero neighborhood $U_{\eta}, A_{\eta} \geq 1$ and $r_{\eta}>0$ such that

$$
u(z) \leq A_{\eta}|\operatorname{Im} z|, z \in V \cap \Gamma\left(\gamma, 1, \eta+\bar{U}_{\eta}, \rho\right) \cap B^{3}\left(0, r_{\eta}\right) .
$$


Because of this and (5.41), a compactness argument implies that we can find $A_{0} \geq 1$, $\delta_{2}>0$ and $0<r_{2}<\rho_{4}$ such that for $K_{1}:=K \cap \mathbb{R}^{3}+\overline{B^{3}\left(0, \delta_{2}\right)}$ we have

$$
u(z) \leq A_{0}|\operatorname{Im} z|, z \in V \cap \Gamma\left(\gamma, 1, K_{1}, \rho\right) \cap B^{3}\left(0, r_{2}\right) .
$$

Since there exists $\mu>0$ such that

$$
|\operatorname{Im} z| \geq \mu|z|, z \in \Gamma(\gamma, 1, K, \rho) \backslash \Gamma\left(\gamma, 1, K_{1}, \rho\right),
$$

the a priori estimate for $u$ and (5.42) imply the existence of $A \geq 1$ and $0<r_{3} \leq r_{2}$, such that

$$
u(z) \leq A|\operatorname{Im} z|, z \in V \cap \Gamma(\gamma, 1, K, \rho) \cap B^{3}\left(0, r_{3}\right) .
$$

Hence $V$ satisfies $\operatorname{PL}(V, \Gamma(\gamma, 1, G, \rho))$ in this case.

If $d>1$, then obvious modifications of the above proof, using (5.31) and Lemma 4.10 (b), give the desired conclusion also in that case.

5.12. Lemma. Let $V$ be an analytic variety in $\mathbb{C}^{n}$ which contains the origin. Assume that for each $\xi \in T_{0} V \cap \mathbb{R}^{n},|\xi|=1$, a convex open zero neighborhood $D_{\xi} \subset B^{n}\left(0, \frac{1}{4}\right)$ is given. Then there exist $m \in \mathbb{N}$ and $\xi_{1}, \ldots, \xi_{m} \in T_{0} V \cap \mathbb{R}^{n},\left|\xi_{j}\right|=1$, and $\eta>0,0<r<\frac{1}{2}$ such that for $S_{j}:=\left\{t \xi_{j}: t>0\right\}$ and $D_{j}:=D_{\xi_{j}}, 1 \leq j \leq m$, the following holds:

$$
V \cap B(0, r) \subset \bigcup_{j=1}^{m} \Gamma\left(S_{j}, 1,2 D_{j}, 1\right) \cup\left\{z \in \mathbb{C}^{n}:|\operatorname{Im} z| \geq \eta|z|\right\} .
$$

Proof. Note first that the following statement can be derived easily from the definition of $T_{0} V$ in 2.1:

For each $\varepsilon>0$ there exists $r(\varepsilon)>0$ such that for each $z \in V \cap$ $B(0, r(\varepsilon))$ there exists $\zeta \in T_{0} V$ satisfying $|z-\zeta| \leq \varepsilon|z|$.

Next let $S:=\left\{x \in \mathbb{R}^{n}:|x|=1\right\}$ and use a compactness argument to find $m \in \mathbb{N}$ and $\xi_{1}, \ldots, \xi_{m} \in T_{0} V \cap \mathbb{R}^{n},\left|\xi_{j}\right|=1$, such that

$$
S \cap T_{0} V \subset \bigcup_{j=1}^{m} \Gamma\left(S_{\xi_{j}}, 1, D_{\xi_{j}}, \infty\right) .
$$

Since $T_{0} V$ is a homogeneous variety, this implies the existence of $\sigma>0$ such that

$$
|\operatorname{Im} \zeta| \geq \sigma|\zeta| \quad \text { for } \quad \zeta \in T_{0} V \backslash \bigcup_{j=1}^{m} \Gamma\left(S_{j}, 1, D_{j}, \infty\right) .
$$

Now choose $\varepsilon>0$ so small that

$$
\varepsilon<\frac{1}{2}, \frac{2 \varepsilon}{1-\varepsilon} \leq \sigma \quad \text { and } \quad D_{j}+B\left(0, \frac{5}{4} \frac{\varepsilon}{1-\varepsilon}\right) \subset 2 D_{j} .
$$

Next choose $0<r<\frac{1}{2}$ so small that $V$ is a closed analytic set in $B(0, r)$ and that $r \leq r(\varepsilon)$, where $r(\varepsilon)$ is the number which exists by (5.43).

Now fix $z \in V \cap B(0, r)$ and assume $|\operatorname{Im} z|<\varepsilon|z|$. Since $r \leq r(\varepsilon)$, (5.43) implies the existence of $\zeta \in T_{0} V$ satisfying

$$
|z-\zeta| \leq \varepsilon|z|
$$

From this we get

$$
(1-\varepsilon)|z| \leq|\zeta| \leq(1+\varepsilon)|z|
$$


and hence by the choice of $\varepsilon$,

$$
|\operatorname{Im} \zeta| \leq|\operatorname{Im} z|+|z-\zeta|<2 \varepsilon|z| \leq \frac{2 \varepsilon}{1-\varepsilon}|\zeta| \leq \sigma|\zeta| .
$$

By (5.44) this implies that for some $j$ we have $\zeta \in \Gamma\left(S_{j}, 1, D_{j}, \infty\right)$. Therefore, there are $t>0$ and $w \in D_{j}$ such that

$$
\zeta=t \xi_{j}+t w
$$

and consequently $z-t \xi_{j}=z-\zeta+t w$. Now note that the hypothesis on $D_{j}$ implies

$$
|z-\zeta| \leq \varepsilon|z| \leq \frac{\varepsilon}{1-\varepsilon}|\zeta| \leq \frac{\varepsilon}{1-\varepsilon}\left(t+\frac{t}{4}\right) \leq \frac{5 \varepsilon}{4(1-\varepsilon)} t .
$$

Hence the choice of $\varepsilon$ gives

$$
z \in t \xi_{j}+t D_{j}+t B\left(0, \frac{5 \varepsilon}{4(1-\varepsilon)}\right) \subset t \xi_{j}+t 2 D_{j} \subset \Gamma\left(S_{j}, 1,2 D_{j}, \infty\right) .
$$

Now note that $D \subset B\left(0, \frac{1}{4}\right)$ implies

$$
|z| \geq|\zeta|-|z-\zeta| \geq t-\frac{1}{4} t-\varepsilon|z|=\frac{3}{4} t-\varepsilon|z| .
$$

Since $|z|<r$, we get from this and the choice of $\varepsilon$ and $r$ that

$$
t<\frac{4}{3}(1+\varepsilon) r<\frac{2}{3}\left(1+\frac{1}{2}\right)=1 .
$$

Hence $z$ belongs to $\Gamma\left(S_{j}, 1,2 D_{j}, 1\right)$. If we let $\eta:=\varepsilon$, then we have shown that each $z \in V \cap B(0, r)$ which satisfies $|\operatorname{Im} z|<\eta|z|$ is in $\bigcup_{j=1}^{m} \Gamma\left(S_{j}, 1,2 D_{j}, 1\right)$, which proves the lemma.

5.13. Lemma. Let $V$ be an analytic variety $V$ in $\mathbb{C}^{n}$ of pure dimension $k \geq 1$ which contains the origin. If $V$ satisfies the following conditions:

(a) $V$ satisfies $\mathrm{RPL}_{\mathrm{loc}}(0)$,

(b) for each $\xi \in T_{0} V \cap \mathbb{R}^{n},|\xi|=1$, there are an open zero neighborhood $G_{\xi}$ in $\mathbb{C}^{n}$ and $\rho_{\xi}>0$ such that $V$ satisfies $\operatorname{PL}\left(V, \Gamma\left(\gamma_{\xi}, 1, G_{\xi}, \rho_{\xi}\right)\right)$ for $\gamma_{\xi}: t \mapsto t \xi$,

then $V$ satisfies $\mathrm{PL}_{\mathrm{loc}}(0)$.

Proof. Choose $0<r<\frac{1}{2}$ such that $V$ is a closed analytic subset of $B(0, r)$. It is no restriction to assume that the sets $G_{\xi}$ in (b) are of the form $3 D_{\xi}$ for some convex open zero neighborhood $D_{\xi} \subset B\left(0, \frac{1}{4}\right)$. Applying Lemma 5.12 we find $0<\rho<r$, $\eta>0, m \in \mathbb{N}$, and $\xi_{1}, \ldots, \xi_{m} \in T_{0} V \cap S^{n-1}$ such that

$$
V \cap B(0, \rho) \subset \bigcup_{j=1}^{m} \Gamma\left(\gamma_{\xi_{j}}, 1,2 D_{\xi_{j}}, 1\right) \cup\left\{z \in \mathbb{C}^{n}:|\operatorname{Im} z| \geq \eta|z|\right\} .
$$

Since $V$ satisfies $\mathrm{RPL}_{\text {loc }}(0)$, we get from [8], Lemma 8, that there is $A_{0}>0$ such that each $u \in \operatorname{PSH}(V \cap B(0, \rho))$ which satisfies

$(\alpha) u(z) \leq 1, z \in V \cap B(0, \rho)$ and

( $\beta) u(z) \leq 0, z \in V \cap B(0, \rho) \cap \mathbb{R}^{3}$

already satisfies

$(\gamma) u(z) \leq A_{0}|z|, z \in V \cap B(0, \rho)$. 
To prove that $V$ satisfies $\mathrm{PL}_{\text {loc }}(0)$, fix any $u \in \operatorname{PSH}(V \cap B(0, \rho))$ which satisfies $(\alpha)$ and $(\beta)$, hence $(\gamma)$. From this and (5.45) we get

$$
u(z) \leq A_{0}|z| \leq \frac{A_{0}}{\eta}|\operatorname{Im} z|, z \in V \cap B(0, \rho) \cap\{z \in \mathbb{C}:|\operatorname{Im} z| \geq \eta|z|\} .
$$

Since $V$ satisfies $\operatorname{PL}\left(V, \Gamma\left(S_{\xi_{j}}, 1,3 D_{\xi_{j}}, r_{\xi}\right)\right)$ for $1 \leq j \leq m$ it follows from Lemma 5.6 that $V$ satisfies $\operatorname{PL}\left(V, \Gamma\left(S_{\xi_{j}}, 1,3 D_{\xi_{j}}, 1\right)\right)$. Since $2 \bar{D}_{\xi_{j}}$ is a compact subset of $3 D_{\xi_{j}}$, this implies that there exist $A_{j}, r_{j}>0$, depending not on $u$, such that for $1 \leq j \leq m$ :

$$
u(z) \leq A_{j} A_{0}|\operatorname{Im} z|, z \in V \cap \Gamma\left(S_{\xi_{j}}, 1,2 \bar{D}_{\xi_{j}}, 1\right) \cap B\left(0, r_{j}\right) .
$$

Now let $A:=A_{0} \max \left\{\frac{1}{\eta}, A_{j}: 1 \leq j \leq m\right\}$ and $r_{0}:=\min \left\{r_{j}: 1 \leq j \leq m\right\}$. Then (5.46) and (5.47) imply that

$$
u(z) \leq A|\operatorname{Im} z|, z \in V \cap B\left(0, r_{0}\right) .
$$

Hence we proved that $V$ satisfies $\mathrm{PL}_{\mathrm{loc}}(0)$.

5.14. Remark. Let $V$ be an analytic variety in $\mathbb{C}^{n}$ which contains the origin, let $\eta \in\left(T_{0} V\right)_{\text {reg }} \cap \mathbb{R}^{n}$ be given and define $\gamma: t \mapsto t \eta$. If $V$ is $(\gamma, 1)$-hyperbolic at $0 \in T_{\gamma, 1} V$, then $V$ is $(\gamma, 1)$-hyperbolic at 0 with respect to each projection $\pi: \mathbb{C}^{n} \rightarrow \mathbb{C}^{n}$ which is noncharacteristic for $T_{\gamma, 1} V$ at 0 and noncharacteristic for $V$ at the origin. In particular, $V$ is 1-hyperbolic at 0 with respect to $\eta$ in the sense of [8], Definition 9. This remark can be proved by the same arguments that we used in the proof of [8], Proposition 12. In that proof, the first part is needed to move to a point in $\left(T_{0} V\right)_{\text {reg }} \cap \mathbb{R}^{n}$, which is now guaranteed by the hypotheses. Note also that the main argument in the proof is the same one that we used to prove Proposition 3.12 .

Proof of Theorem 5.3. (a) $\Longrightarrow$ (b): Apply Theorem 3.13 with $n=3, k=2$.

(b) $\Longrightarrow$ (c): The first condition in (c) follows from (i) in 3.13(b) and 2.7(b). All the other conditions are a subset of those in 3.13(b).

(c) $\Longrightarrow$ (a): To apply Lemma 5.13 , note first that $V$ satisfies $\operatorname{RPL}_{\text {loc }}(0)$. This follows from [8], Theorem 10, if we show that for each irreducible component $W$ of $T_{0} V$ there is $\eta \in W \cap \mathbb{R}^{3}$ such that $V$ is 1-hyperbolic at 0 with respect to $\eta$ and $-\eta$ in the sense of [8], Definition 9 . Since $T_{0} V$ and hence $T_{0} W$ satisfy $\mathrm{PL}_{\text {loc }}(0)$ by hypothesis, $\left(T_{0} V\right)_{\text {reg }} \cap \mathbb{R}^{3} \cap\left(T_{0} W\right)_{\text {reg }} \neq \emptyset$. Therefore, the hypotheses in 5.3(c) together with Remark 5.14 imply that the hypotheses of 8 , Theorem 10 , are fulfilled so that $V$ satisfies $\mathrm{RPL}_{\text {loc }}(0)$.

To show that the second condition in Lemma 5.13 is also fulfilled, fix $\zeta \in S^{2}$ and distinguish the following cases:

Case 1: $\eta \in\left(T_{0} V\right)_{\text {reg }} \cap S^{2}$. Then $V$ is $\left(\gamma_{\zeta}, 1\right)$-hyperbolic in $\eta-\zeta \in T_{\gamma_{\zeta}, 1} V$ by hypothesis. Therefore, Lemma 5.7 implies the existence of a zero neighborhood $G$ in $\mathbb{C}^{3}$ and of $r>0$ such that $V$ satisfies $\operatorname{PL}\left(V, \Gamma\left(\gamma_{\zeta}, 1, \eta-\zeta+G, r\right)\right)$. Since $\Gamma\left(\gamma_{\zeta}, 1, \eta-\zeta+G, r\right)=\Gamma\left(\gamma_{\eta}, 1, G, r\right)$ this proves that condition 5.13(b) holds for $\eta$.

Case 2: $\eta \in\left(T_{0} V\right)_{\operatorname{sing}} \cap S^{2}$ and $\eta-\zeta$ is a simple point of $T_{\gamma_{\zeta}, 1} V$. Since $T_{0} V$ satisfies $\mathrm{PL}_{\mathrm{loc}}(0)$ by hypothesis, it follows from Theorem 2.7(b) and Proposition 3.5 that $T_{\gamma_{\eta}, 1}\left(T_{0} V\right)=T_{0}\left(T_{0} V\right)-\eta=T_{0} V-\eta$ satisfies $\mathrm{PL}_{\text {loc }}(0)$. Hence $T_{0} V$ satisfies $\mathrm{PL}_{\text {loc }}(\eta)$. Therefore, Braun [5], Corollary 12, shows that $T_{\gamma_{\zeta}, 1} V=T_{0} V-\zeta$ is locally hyperbolic at $\eta-\zeta$. By the homogeneity of $T_{0} V$, the hypotheses of 5.3 (c) imply that $V$ is $\left(\gamma_{\zeta}, 1\right)$-hyperbolic at each $\kappa \in\left(T_{\gamma_{\zeta}, 1} V\right)_{\text {reg }} \cap \mathbb{R}^{3}$. Consequently, $V$ 
is $\left(\gamma_{\zeta}, 1\right)$-hyperbolic at $\eta-\zeta$, by Lemma 5.9. Therefore it follows as in Case 1 that the condition in 5.13(b) holds for $\eta$.

Case 3: $\eta \in\left(T_{0} V\right)_{\text {sing }} \cap S^{2}$ and $\eta-\zeta$ is not a simple point of $T_{\gamma_{\zeta}, 1} V$. By the remark following Lemma 4.9, the present hypothesis implies that $\eta$ belongs to the set $M_{1}$, defined in section 5.1 and that for $A(\eta):=T_{\eta}\left(T_{0} V\right) \cup T_{0} V$, the pair $\left(\sigma_{\eta}, \Delta^{A(\eta)}\left(\sigma_{\eta}, 1\right)\right)$ belongs to $\mathcal{C}_{1}$. In particular $\mathcal{C} \neq \emptyset$ and by section 5.1 there exists $N \in \mathbb{N}$ such that $\mathcal{C}=\bigcup_{j=1}^{N} \mathcal{C}_{j}$. We claim that the following assertion holds:

For each $1 \leq j \leq N$, each $(\gamma, d) \in \mathcal{C}_{j}$, and each $\xi \in T_{\gamma, d} V \cap \mathbb{R}^{3}$ there exist an open zero neighborhood $D_{\xi}$ and $\rho_{\xi}>0$ such that $V$ satisfies $\operatorname{PL}\left(V, \Gamma\left(\gamma, d, D_{\xi}, \rho_{\xi}\right)\right)$.

If (5.48) holds, then we can argue as follows: By the remark following Lemma 4.9, $0 \in T_{\sigma_{\eta}, 1} V$ is not a simple point of $T_{\sigma_{\eta}, 1} V=T_{0} V-\eta$. By the arguments in case 2, $V$ is locally hyperbolic at each real point of $T_{\sigma_{\eta}, 1} V$ and $\left(\sigma_{\eta}, 1\right)$-hyperbolic at each real regular point of $T_{\sigma_{\eta}, 1} V \cap \mathbb{R}^{3}$. Hence the conditions (a)-(c) of Lemma 5.11 are fulfilled. By (5.48), also condition 5.11(d) holds. Hence this lemma implies the existence of a zero neighborhood $G$ and $\rho>0$ such that $V$ satisfies $\operatorname{PL}\left(V, \Gamma\left(\sigma_{\eta}, 1, G, \rho\right)\right)$. Consequently, condition 5.13(b) holds for $\eta$ also in this case.

This shows that, by Lemma 5.13, $V$ satisfies $\mathrm{PL}_{\text {loc }}(0)$ if we prove (5.48). To do so we argue by induction downward from $N$ to 1 . To start the induction, note that by (5.2) for each $(\gamma, d) \in \mathcal{C}_{N}$ all points in $\left(T_{\gamma, d} V\right)_{\operatorname{sing}} \cap \mathbb{R}^{3}$ are simple points. Since the definition of $\mathcal{C}$ in section 5.1 and the hypotheses in 5.3(c) imply that for each $\xi \in T_{\gamma, d} V \cap \mathbb{R}^{3}$ the hypotheses of Lemma 5.9 are fulfilled, we conclude from this lemma and Lemma 5.7 that (5.48) holds for $j=N$.

Assume now that (5.48) holds for $j+1$, where $2 \leq j+1 \leq N$. To show that (5.48) also holds for $j$, fix $(\gamma, d) \in \mathcal{C}_{j}$ and $\eta \in T_{\gamma, d} V \cap \mathbb{R}^{3}$. If $\eta$ is a regular point of $T_{\gamma, d} V$, then the hypothesis (ii) in 5.3(c) together with Lemma 5.7 implies that (5.48) holds for $\eta$. If $\eta \in\left(T_{\gamma, d} V\right)_{\operatorname{sing}} \cap \mathbb{R}^{3}$ is a simple point of $T_{\gamma, d} V$, then condition 5.3(c)(i) together with Theorem 2.7(c) and Proposition 3.16 implies that $T_{\gamma, d} V$ is locally hyperbolic at $\eta$. By condition (ii) of 5.3(c), the second condition in Lemma 5.9 is also fulfilled. Hence we get from Lemma 5.9 that $V$ is $(\gamma, d)$-hyperbolic at $\eta$. By Lemma 5.7, this implies that (5.48) holds for $\eta$ in this case.

If $\eta \in\left(T_{\gamma, d} V\right)_{\text {sing }} \cap \mathbb{R}^{3}$ is not a simple point of $T_{\gamma, d} V$, then it follows from Lemma 4.9(b) that there are $\zeta \in M_{\gamma, d}$ and $\tau \in \mathbb{R}$ such that $\eta=\zeta+\tau \xi_{0}$, where $\xi_{0}$ is the tangent vector of $\gamma$ at zero. First assume $\tau=0$. In order to apply Lemma 5.11, define $\gamma_{\zeta}$ as in section 5.1 by $\gamma_{\zeta}(t):=\gamma(t)+\zeta t^{d}$. Then it is easy to check that $T_{\gamma_{\zeta}, d} V=T_{\gamma, d} V-\zeta$. Hence 0 is not a simple point of $T_{\gamma_{\zeta}, d} V$ and consequently condition 5.11(a) holds. By the same reason, the conditions 5.11(b) and (c) follow as before from the conditions 5.3(c)(i) and (ii). Since $T_{0}\left(T_{\gamma_{\zeta}, d} V\right)=T_{\zeta}\left(T_{\gamma, d} V\right)$, we have $A(\gamma, \zeta)=A(\sigma, 0)$ in the notation of section 5.1. Hence $\Delta^{A(\gamma, \zeta)}(\gamma, d)=$ $\Delta^{A\left(\gamma_{\zeta}, 0\right)}\left(\gamma_{\zeta}, d\right)=: \Delta$. Since $\left(\gamma_{\zeta}, \Delta\right) \in \mathcal{C}_{j+1}$ by the definition of $\mathcal{C}_{j+1}$, the induction hypothesis implies that for each $\xi \in T_{\gamma_{\zeta}, \Delta} V \cap \mathbb{R}^{3}$, there are a zero neighborhood $G_{\xi}$ and $\rho_{\xi}>0$ such that $V$ satisfies $\operatorname{PL}\left(V, \Gamma\left(\gamma_{\zeta}, \Delta, \xi+G_{\xi}, \rho_{\xi}\right)\right)$. Therefore, the conditions (a)-(d) of Lemma 5.11 are fulfilled. Hence this lemma implies the existence of a zero neighborhood $G$ and $\rho>0$ such that $V$ satisfies $\operatorname{PL}\left(V, \Gamma\left(\gamma_{\zeta}, d, G, \rho\right)\right)$. Since $\Gamma\left(\gamma_{\zeta}, d, G, \rho\right)=\Gamma(\gamma, d, \zeta+G, \rho), V$ satisfies $\operatorname{PL}(V, \Gamma(\gamma, d, \zeta+G, \rho))$.

Now consider $\eta=\zeta+\tau \xi_{0}$ for arbitrary $\tau \in \mathbb{R}$. Then it follows from (4.2) that there are a zero neighborhood $D_{\eta}$ and $\rho_{\eta}>0$ such that $\Gamma\left(\gamma, d, \eta+D_{\eta}, \rho_{\eta}\right) \subset$ 
$\Gamma(\gamma, d, \zeta+G, \rho)$. Hence it follows from Lemma 5.6(a) that (5.48) holds for $\eta$ also in this case.

\section{Application to partial differential equations}

In this section we are going to apply our main theorem together with a result of Hörmander [17], to derive a new characterization of the linear partial differential operators with constant coefficients which are surjective on the space $\mathcal{A}\left(\mathbb{R}^{4}\right)$ of real analytic functions on $\mathbb{R}^{4}$. The first step to do this is the follow lemma.

6.1. Lemma. Let $P \in \mathbb{C}\left[z_{1}, \ldots, z_{n+1}\right]$ be homogeneous and let $\xi \in V(P) \cap \mathbb{R}^{n+1}, \xi \neq$ 0 , be given. Choose $\xi_{1}, \ldots, \xi_{n} \in \mathbb{R}^{n+1}$ such that $\left\{\xi_{1}, \ldots, \xi_{n}, \xi\right\}$ is a basis of $\mathbb{R}^{n+1}$ and define

$$
Q\left(z^{\prime}\right):=P\left(\sum_{j=1}^{n} z_{j} \xi_{j}+\xi\right), z^{\prime}:=\left(z_{1}, \ldots, z_{n}\right) \in \mathbb{C}^{n} .
$$

Then $V(P)$ satisfies $\mathrm{PL}_{\mathrm{loc}}(\xi)$ if and only if $V(Q) \subset \mathbb{C}^{n}$ satisfies $\mathrm{PL}_{\mathrm{loc}}(0)$.

Proof. It is no restriction to assume that $\xi=(0, \ldots, 0,1)$ and $Q\left(z^{\prime}\right)=P\left(z^{\prime}, 1\right)$. Since $P$ is homogeneous, the map

$$
F: V(P) \cap\left(B^{n}(0,1) \times B^{1}\left(1, \frac{1}{2}\right)\right) \rightarrow V(Q), \quad F\left(z^{\prime}, z_{n+1}\right):=\frac{z^{\prime}}{z_{n+1}}
$$

has, indeed, values in $V(Q)$.

Assume now that $V(P)$ satisfies $\mathrm{PL}_{\mathrm{loc}}(\xi)$. Then Lemma 3.3 implies that there exist $A>0$ and $0<r<1$ such that each $u$ which is plurisubharmonic on the set $B:=V(P) \cap\left(B^{n}(0,1) \times B^{1}\left(1, \frac{1}{2}\right)\right)$ and satisfies

$$
u(z) \leq 1, z \in B \quad \text { and } \quad u(z) \leq 0, z \in B \cap \mathbb{R}^{n+1}
$$

also satisfies

$$
u(z) \leq A|\operatorname{Im} z|, z \in V(P) \cap B^{n+1}(\xi, r) .
$$

Now fix $v \in \operatorname{PSH}\left(V(Q) \cap B^{n}(0,2)\right)$ which satisfies the conditions $(\alpha)$ and $(\beta)$ of Definition 3.1. Then note that $F(B) \subset B^{n}(0,2)$. Hence $u:=v \circ F$ is plurisubharmonic on $B$ and satisfies $u(z) \leq 1$ for $z \in B$. For $z \in V \cap B \cap \mathbb{R}^{n+1}, F(z)$ is in $V(Q) \cap \mathbb{R}^{n} \cap B(0,2)$, hence the condition $(\beta)$ for $v$ implies $u(z) \leq 0$. Consequently, we have

$$
u(z) \leq A|\operatorname{Im} z|, \quad z \in V(P) \cap B^{n+1}(\xi, r) .
$$

By the definition of $u$, this implies in particular

$$
A\left|\operatorname{Im} z^{\prime}\right| \geq u\left(z^{\prime}, 1\right)=v\left(F\left(z^{\prime}, 1\right)\right)=v\left(z^{\prime}\right), \quad z^{\prime} \in B^{n}(0, r) .
$$

Hence $V(Q)$ satisfies $\mathrm{PL}_{\mathrm{loc}}(0)$.

To prove the converse implication, fix $x_{n+1} \in B^{1}\left(1, \frac{1}{4}\right) \cap \mathbb{R}$ and note that the map

$$
G: V(Q) \rightarrow V(P), \quad G\left(z^{\prime}\right):=\left(x_{n+1} z^{\prime}, x_{n+1}\right)
$$

has, indeed, values in $V(P)$. Since $V(Q)$ satisfies $\mathrm{PL}_{\text {loc }}(0)$, we get from Lemma 3.3 that there exist $A_{0}>0$ and $0<r_{0}<1 / 2$ such that $3.1(\gamma)$ holds for all functions $\varphi$ on $V(Q) \cap B^{n}\left(0, \frac{1}{2}\right)$ which satisfy $3.1(\alpha)$ and $3.1(\beta)$. Now let $u \in$ $\operatorname{PSH}\left(V(P) \cap B^{n+1}(\xi, 1)\right)$ be given and note that $G\left(V(Q) \cap B^{n}\left(0, \frac{1}{2}\right)\right) \subset B^{n+1}(\xi, 1)$. Hence

$$
\varphi\left(z^{\prime}\right):=u\left(G\left(z^{\prime}\right)\right), z^{\prime} \in V(Q) \cap B^{n}\left(0, \frac{1}{2}\right)
$$


is plurisubharmonic and satisfies the conditions $3.1(\alpha)$ and $(\beta)$ on $B^{n}\left(0, \frac{1}{2}\right)$. Hence it also satisfies

$$
\varphi\left(z^{\prime}\right) \leq A_{0}\left|\operatorname{Im} z^{\prime}\right|, \quad z^{\prime} \in V(Q) \cap B^{n}\left(0, r_{0}\right) .
$$

Next note that for $w \in B^{n}\left(0, \frac{r_{0}}{4}\right)$ we have $\left|w / x_{n+1}\right|<r_{0} / 3<r_{0}$. Hence the definition of $\varphi$ and the previous estimate imply

$$
u\left(w, x_{n+1}\right)=\varphi\left(\frac{w}{x_{n+1}}\right) \leq A_{0}\left|\operatorname{Im} \frac{w}{x_{n+1}}\right| .
$$

Consequently, since $1 / x_{n+1} \leq \frac{4}{3}$,

$$
u\left(w, x_{n+1}\right) \leq \frac{4}{3} A_{0}|\operatorname{Im} w|, \quad\left(w, x_{n+1}\right) \in V(P) \cap\left(B^{n}\left(0, \frac{r_{0}}{4}\right) \times B^{1}\left(1, \frac{1}{4}\right)\right) .
$$

Next fix $z=\left(z^{\prime}, z_{n+1}\right) \in V(P) \cap B^{n}\left(0, \frac{r_{0}}{8}\right) \times B^{1}\left(1, \frac{1}{8}\right)$, let $w:=z^{\prime} / z_{n+1}$ and note that $|w| \leq \frac{r_{0}}{8} \cdot \frac{9}{8}$. Since $P$ is homogeneous, $(\lambda w, \lambda)$ belongs to $V(P) \cap\left(B^{n}\left(0, \frac{r_{0}}{4}\right) \times\right.$ $\left.B^{1}\left(1, \frac{1}{4}\right)\right)$ for each $\lambda \in B\left(1, \frac{1}{4}\right)$. Hence the function

$$
\chi: B^{1}\left(1, \frac{1}{4}\right) \rightarrow\left[-\infty, \infty\left[, \chi(\lambda):=u(\lambda w, \lambda)-A_{0}|\operatorname{Im} w|\right.\right.
$$

is bounded above by 1 . The previous estimate shows that $\chi(\lambda) \leq 0$ for $\lambda \in$ $B^{1}\left(1, \frac{1}{4}\right) \cap \mathbb{R}$. Hence a standard argument, using estimates for the harmonic measure of the half disk, implies the existence of $A_{1} \geq 1$, not depending on $u$, such that

$$
\chi(\lambda) \leq A_{1}|\operatorname{Im} \lambda|, \quad \lambda \in B^{1}\left(1, \frac{1}{8}\right) .
$$

From this estimate and the definition of $\chi$ it follows that

$$
u(z)=u\left(z_{n+1} w, z_{n+1}\right)=\chi\left(z_{n+1}\right)+A_{0}|\operatorname{Im} w| \leq A_{1}\left|\operatorname{Im} z_{n+1}\right|+A_{0}|\operatorname{Im} w| .
$$

To estimate this further, note that

$$
|\operatorname{Im} w|=\left|\operatorname{Im} \frac{z^{\prime}}{z_{n+1}}\right| \leq \sqrt{3}\left|\operatorname{Im} z^{\prime}\right|+2\left|\operatorname{Im} z_{n+1}\right| .
$$

Hence we get for $z \in V(P) \cap\left(B^{n}\left(0, \frac{r_{0}}{8}\right) \times B^{1}\left(1, \frac{1}{8}\right)\right)$ that

$$
u(z) \leq A_{0} \sqrt{3}\left|\operatorname{Im} z^{\prime}\right|+\left(2 A_{0}+A_{1}\right)\left|\operatorname{Im} z_{n+1}\right| \leq C|\operatorname{Im} z|,
$$

where $C$ does not depend on $u$. Obviously, this implies that $V(P)$ satisfies $\mathrm{PL}_{\text {loc }}(\xi)$.

6.2. Definition. Under the hypotheses of Lemma 6.1 we call the polynomial $Q$ defined there a reduction of $P$ at $\xi$. Note that there are many reductions of $P$ at $\xi$, however, Lemma 6.1 shows that each can be used to decide whether $V(P)$ satisfies $\mathrm{PL}_{\mathrm{loc}}(\xi)$.

6.3. The Whitney cone $C_{4}(V, \xi)$. Let $V \subset \mathbb{C}^{n}$ be an analytic set and let $\xi \in V$. Then the Whitney cone $C_{4}(V, \xi)$ consists of all those $v \in \mathbb{C}^{n}$ for which there are sequences $\left(z_{j}\right)_{j \in \mathbb{N}}$ in $V$ and $\left(v_{j}\right)_{j \in \mathbb{N}}$ in $\mathbb{C}^{n}$ such that $\lim _{j \rightarrow \infty} z_{j}=\xi$ and $\lim _{j \rightarrow \infty} v_{j}=$ $v$ and, for each $j$, the point $z_{j}$ is a regular point of $V$ and $v_{j} \in T_{z_{j}} V$.

6.4. Remark. If $V$ is analytic of pure dimension $k$, then $\operatorname{dim} C_{4}(V, \xi) \geq k$ for each $\xi \in V$ (see Chirka 14, 9.2). Furthermore, the set

$$
W:=\left\{\xi \in V: \operatorname{dim} C_{4}(V, \xi)>k\right\}
$$

is analytic of dimension not exceeding $k-2$ ([14], Lemma 9.4). In particular, if $V$ is a homogeneous algebraic hypersurface in $\mathbb{C}^{4}$, then $W$ consists of a discrete set of 
complex lines. Since these can accumulate nowhere, $W$ consists of a finite number of complex lines, and thus is algebraic. Using algorithmic commutative algebra, it is also possible to construct explicit polynomial equations for $W$ from a set of polynomials defining $V$. For a detailed explanation we refer to [10].

One more fact, proved by Chirka in [14], 9.5, Proposition 2, about $C_{4}(V, \xi)$ will be used repeatedly: If $V$ is analytic of pure dimension $k$ and irreducible in $\xi \in V_{\text {sing }}$ and if $\operatorname{dim} C_{4}(V, \xi)=k$, then the dimension of $V_{\text {sing }}$ in $\xi$ is $k-1$.

6.5. A partition of $V(P)$. For homogeneous $P \in \mathbb{C}\left[Z_{1}, \ldots, Z_{4}\right]$, we partition $V(P)$ into the following sets $V_{d}, 0 \leq d \leq 3$ :

(a) $V_{3}$ is the set of regular points of $V(P) \backslash\{0\}$,

(b) $V_{2}:=\left\{z \in V(P)_{\text {sing }}: z \neq 0, V(P)_{\text {sing }}\right.$ regular in $\left.z, \operatorname{dim} C_{4}(V(P), z)=3\right\}$,

(c) $V_{1}:=V(P)_{\text {sing }} \backslash\left(V_{2} \cup\{0\}\right)$,

(d) $V_{0}:=\{0\}$.

6.6. Lemma. Let $0 \leq d \leq 3$ :

(a) $V_{d}$ and $V_{d} \cap \mathbb{R}^{4}$ both have at most a finite number of connected components.

(b) $V_{d}$ is either empty or $\operatorname{dim} V_{d}=d$.

Proof. $V_{d}$ and $V_{d} \cap \mathbb{R}^{4}$ are semi-algebraic, hence admit a triangulation (see Bochnak, Coste, and Roy [3], Théorème 9.2.1). This proves (a).

(b) is immediate for $d=0$ and $d=3$. If $\xi \in V_{1}$, then $\xi \in\left(V(P)_{\text {sing }}\right)_{\text {sing }}$ or $\operatorname{dim} C_{4}(V(P), \xi)=4$. Using Remark 6.4 the claim follows for the case $d=1$. To treat the remaining case, fix $\xi \in V_{2}$. It suffices to show that the dimension of $V(P)_{\text {sing }}$ in $\xi$ is 2 . To do so, let $[V(P)]_{\xi}=\left[Z_{1}\right]_{\xi} \cup \cdots \cup\left[Z_{l}\right]_{\xi}$ be a decomposition of the germ into irreducible components. Assume first that all $Z_{j}$ are regular in $\xi$. Then $l \geq 2$ and $\operatorname{dim} Z_{1} \cap Z_{2}=2$. Since $Z_{1} \cap Z_{2} \subset V(P)_{\text {sing }}$, the claim is shown for this case. Now assume that there is $j$ such that $Z_{j}$ is singular in $\xi$. Then $\operatorname{dim} C_{4}\left(Z_{j}, \xi\right)=3$ since Whitney cones of type $C_{4}$ are additive by [14], 9.1, statement 3. Then Remark 6.4 shows that the dimension of $\left(Z_{j}\right)_{\operatorname{sing}}$ in $\xi$ is 2 .

6.7. Proposition. Let $P \in \mathbb{C}\left[Z_{1}, \ldots, Z_{4}\right]$ be homogeneous and let $d \in\{1,2,3\}$. The set of points $\xi \in V_{d} \cap \mathbb{R}^{4}$ such that $V(P)$ satisfies $\mathrm{PL}_{\mathrm{loc}}(\xi)$ is closed in $V_{d} \cap \mathbb{R}^{4}$.

Proof. Pick $\xi \in V_{d} \cap \mathbb{R}^{4}$ such that $\mathrm{PL}_{\text {loc }}(\xi)$ does not hold. Fix $\xi_{1}, \xi_{2}, \xi_{3} \in \mathbb{R}^{4}$ such that $\left(\xi_{1}, \xi_{2}, \xi_{3}, \xi\right)$ is a basis of $\mathbb{R}^{4}$, let $Q$ be as in Lemma 6.1, and define

$$
W_{d}:=\left\{\left(\zeta_{1}, \zeta_{2}, \zeta_{3}\right) \in V(Q): \xi+\sum_{j=1}^{3} \zeta_{j} \xi_{j} \in V_{d}\right\} .
$$

Since $P$ is homogeneous, $V(P)$ satisfies $\mathrm{PL}_{\text {loc }}(\xi)$ at $\xi \in V(P) \cap \mathbb{R}^{4}, \xi \neq 0$, if and only if $V(P)$ satisfies $\mathrm{PL}_{\mathrm{loc}}(t \xi)$ at $t \xi$ for all $t>0$. Hence Lemma 6.1 implies that it suffices to consider $W_{d}$ in a neighborhood of zero instead of $V_{d}$ in a neighborhood of $\xi$.

Consider the case $d=3$ first. Then locally near zero, $W_{3}$ is a graph, i.e., there are neighborhoods of zero $U \subset \mathbb{C}^{3}$ and $U_{1} \subset \mathbb{C}^{2}$ and a holomorphic function $f$ such that

$$
W_{3} \cap U=\left\{\left(z_{1}, z_{2}, f\left(z_{1}, z_{2}\right)\right):\left(z_{1}, z_{2}\right) \in U_{1}\right\}
$$

in suitable coordinates. Since $V(Q)$ does not satisfy $\mathrm{PL}_{\text {loc }}(0)$, Hörmander [17], Theorem 6.3 and proof of Theorem 6.5, imply that not all Taylor coefficients of $f$ 
are real. Since the description (6.1) holds in a whole neighborhood of zero, the claim is proved for $d=3$.

Since $W_{1}$ is finite there is nothing to prove for $d=1$.

Finally consider the case $d=2$. Then there is at least one component $[Z]_{0}$ of the germ $[V(Q)]_{0}$ which does not satisfy $\mathrm{PL}_{\text {loc }}(0)$. Let us first treat the case that 0 is a regular point of $Z$. Then the argument used to treat $d=3$ shows that there is whole neighborhood $U$ of 0 such that $Z$ does not satisfy $\operatorname{PL}_{\text {loc }}(\zeta)$ for any $\zeta \in Z \cap U \cap \mathbb{R}^{3}$. Since $V(Q)$ is singular in 0 there must be an additional component of $[V(Q)]_{0}$, which we will denote by $\left[Z_{1}\right]_{0}$. Then $Z \cap Z_{1} \subset V(Q)_{\text {sing. Note that }}$ $\operatorname{dim} Z \cap Z_{1}=1 \geq \operatorname{dim} V(Q)_{\text {sing }}$ and that $V(Q)_{\text {sing }}$ is regular in 0 since $0 \in W_{2}$. Hence $Z \cap Z_{1}$ and $V(Q)_{\text {sing }}$ coincide near 0 . In particular, $W_{2} \subset Z$ locally near 0 , and the claim follows from the regularity of $Z$ in 0 .

Now let 0 be a singular point of $Z$. Then $2 \leq \operatorname{dim} C_{4}(Z, 0) \leq \operatorname{dim} C_{4}(V, \xi)-1=2$ and hence, by Remark [6.4, the dimension of $Z_{\text {sing }}$ in 0 is 1 . Then $\left[Z_{\text {sing }}\right]_{0}=$ $\left[V(Q)_{\text {sing }}\right]_{0}$ since $\left[V(Q)_{\text {sing }}\right]_{0}$ is regular and has dimension 1 . Hence $\left[Z_{\text {sing }}\right]_{0}$ is also regular, and there is a neighborhood $U$ of 0 such that each $\zeta \in Z_{\text {sing }} \cap U$ is a regular point of $Z_{\text {sing }}$ satisfying $\operatorname{dim} C_{4}(Z, \zeta)=2$ and such that $Z$ is irreducible in $\zeta$. Now the proof of Braun [5], Theorem 11, shows that $Z$ does not satisfy $\mathrm{PL}_{\text {loc }}(\zeta)$ for any $\zeta \in Z_{\text {sing }} \cap U \cap \mathbb{R}^{4}$.

Remark. The main geometrical ingredient in the proof of Braun 5, Theorem 11, is Theorem 4.2 of Stutz 31: Let $Z \subset \mathbb{C}^{n}$ be an analytic variety of codimension 1 which is irreducible in $z \in\left(Z_{\text {sing }}\right)_{\text {reg }}$, assume $\operatorname{dim} C_{4}(Z, z)=n-1$, and let $L$ be a hyperplane transversal to $Z_{\text {sing }}$ in $z$. Then it is clear that $Z \cap L$ is a curve that can be described by a Puiseux series. Stutz proved in this context that the parameters in this Puiseux series vary analytically with $w \in Z_{\text {sing }} \cap U$, where $U$ is a suitable neighborhood of $z$. Hence Stutz's theorem is a result about equisingularity in the spirit of Zariski [35. Thus the singularity type is constant in the connected components of $V_{2}$. The same holds for $V_{3}$, since all regular points are alike, and for $V_{1}$, since $V_{1} \cup\{0\}$ is a finite union of lines.

To apply our results to the characterization of surjective partial differential operators on $\mathcal{A}\left(\mathbb{R}^{4}\right)$, the space of all complex valued real analytic functions on $\mathbb{R}^{4}$, we need a few notations:

6.8. Notation. Let $P \in \mathbb{C}\left[Z_{1}, \ldots, Z_{4}\right]$ be a polynomial and let $P_{m}$ be its principal part. If $P=\sum_{|\alpha| \leq m} a_{\alpha} z^{\alpha}$, then the corresponding partial differential operator is defined by

$$
P(D):=\sum_{|\alpha| \leq m} i^{-|\alpha|} \frac{\partial^{\alpha}}{\partial z^{\alpha}}
$$

Let

$$
V\left(P_{m}\right)=\bigcup_{d=0}^{3} V_{d}
$$

be the partition of the variety $V\left(P_{m}\right)$ of the principal part defined in section 6.5 For $1 \leq d \leq 3$ let $Z_{d, 1}, \ldots, Z_{d, N_{d}}$ be the connected components of $V_{d} \cap \mathbb{R}^{4}$.

6.9. Theorem. Let $P \in \mathbb{C}\left[Z_{1}, \ldots, Z_{4}\right]$ be a polynomial and let $P_{m}$ be its principal part. The following are equivalent:

(a) $P(D): \mathcal{A}\left(\mathbb{R}^{4}\right) \rightarrow \mathcal{A}\left(\mathbb{R}^{4}\right)$ is surjective. 
(b) $V\left(P_{m}\right)$ satisfies $\mathrm{PL}_{\mathrm{loc}}(\xi)$ for each $\xi \in V\left(P_{m}\right) \cap \mathbb{R}^{4} \backslash\{0\}$.

(c) For each $d \in\{1,2,3\}$ and each $j \in\left\{1, \ldots, N_{d}\right\}$ there is $\xi \in Z_{d, j}$ such that $V\left(P_{m}\right)$ satisfies $\mathrm{PL}_{\mathrm{loc}}(\xi)$.

(d) For each $\xi \in V\left(P_{m}\right) \cap \mathbb{R}^{4} \backslash\{0\}$ a reduction of $P_{m}$ at $\xi$ is hyperbolic in conoids at the origin.

(e) For each $d \in\{1,2,3\}$ and each $j \in\left\{1, \ldots, N_{d}\right\}$ there is $\xi \in Z_{d, j}$ such that a reduction of $P_{m}$ at $\xi$ is hyperbolic in conoids at the origin.

Proof. The equivalence of (a) and (b) was proved by Hörmander 17, 1.1, 1.2, and 2.1. The set of points $\xi \in V_{d} \cap \mathbb{R}^{4}$ for which $V\left(P_{m}\right)$ satisfies $\mathrm{PL}_{\text {loc }}(\xi)$ is open in $V\left(P_{m}\right) \cap \mathbb{R}^{4}$ by Lemma 3.3 and closed in $V_{d} \cap \mathbb{R}^{4}$ by Proposition 6.7. This shows the equivalence of (b) and (c). Theorem 5.3 and Lemma 6.1 imply the equivalence of (b) and (d) and of (c) and (e), respectively.

6.10. Corollary. For $P$ and $P_{m}$ as in Theorem 6.9 and for each convex open set $\Omega$ in $\mathbb{R}^{4}$ the surjectivity of $P(D): \mathcal{A}(\Omega) \rightarrow \mathcal{A}(\Omega)$ implies that the equivalent conditions in Theorem 6.9 hold.

Proof. This follows from Hörmander [17, since the surjectivity of $P(D): \mathcal{A}(\Omega) \rightarrow$ $\mathcal{A}(\Omega)$ implies the surjectivity of $P(D): \mathcal{A}\left(\mathbb{R}^{4}\right) \rightarrow \mathcal{A}\left(\mathbb{R}^{4}\right)$.

6.11. Remark. Define the homogeneous polynomial $P \in \mathbb{C}\left[z_{1}, \ldots, z_{4}\right]$ by

$$
P\left(x_{1}, \ldots, x_{4}\right):=x_{1}^{2}\left(x_{2}^{2}\left(x_{3}^{2}+x_{4}^{2}\right)+x_{3}^{4}\right)+x_{2}^{6}+x_{3}^{6}+x_{4}^{6} .
$$

Using localizations at infinity, Langenbruch [20], p. 256, showed that for the half space $\Omega_{4}:=\left\{x \in \mathbb{R}^{4}: x_{4}>0\right\}$ the operator $P(D): \mathcal{A}(\Omega) \rightarrow \mathcal{A}(\Omega)$ is not surjective. In Example 7.8 below, we show that $P$ fails the conditions in Theorem 6.9] Hence for each convex open set $\Omega$ in $\mathbb{R}^{4}$, prove that $P(D): \mathcal{A}(\Omega) \rightarrow \mathcal{A}(\Omega)$ fails to be surjective, by Corollary 6.10

\section{FURTHER RESULTS AND EXAMPLES}

In this section we prove the theorem that is stated in the Introduction and we treat several examples in order to show how our main results in sections 5 and 6 can be applied. To do this we first indicate that Theorem 5.3 can be interpreted in an algorithmic way and that it simplifies considerably if all relevant limit varieties have multiplicity one.

7.1. An algorithm. Let $V$ be an analytic surface in $\mathbb{C}^{3}$ which contains the origin. We now show that Theorem 5.3 provides an algorithm that decides after finitely many steps whether $V$ satisfies $\mathrm{PL}_{\text {loc }}(0)$.

(1) If $T_{0} V$ does not satisfy $\mathrm{PL}_{\text {loc }}(0)$, then $V$ does not satisfy $\mathrm{PL}_{\text {loc }}(0)$ by Proposition 3.5. Otherwise determine $\left(T_{0} V\right)_{\operatorname{sing}} \cap S^{2}$, fix $\xi \in S^{2}$ as in section 5.1, let $\gamma_{\xi}: t \mapsto t \xi$, and consider (2).

(2) If there exists $\eta \in\left(T_{0} V\right)_{\text {reg }} \cap S^{2}$ such that $V$ is not $\left(\gamma_{\xi}, 1\right)$-hyperbolic at $\eta-\xi$, then $V$ does not satisfy $\mathrm{PL}_{\text {loc }}(0)$ by Theorem 5.3. Otherwise consider $(3)$.

(3) If for each $\eta \in\left(T_{0} V\right)_{\text {sing }} \cap S^{2}, \eta-\xi$ is a simple point of $T_{\gamma_{\xi}, 1} V$, then $V$ satisfies $\mathrm{PL}_{\text {loc }}(0)$, because the construction of $\mathcal{C}$ in section 5.1 shows that $\mathcal{C}=\emptyset$. Therefore it follows from (1) and (2) that the hypotheses of Theorem $5.3(\mathrm{c})$ are fulfilled. Thus $V$ satisfies $\mathrm{PL}_{\mathrm{loc}}(0)$. 
Otherwise let

$$
M_{1}:=\left\{\eta \in\left(T_{0} V\right)_{\operatorname{sing}} \cap S^{2}, \eta-\xi \text { is not simple }\right\} .
$$

For $\eta \in M_{1}$ let $\gamma_{\eta}: t \mapsto t \eta$ and $A(\eta):=T_{\eta}\left(T_{0} V\right) \cup T_{0} V$. Determine the critical level $\Delta_{\eta}:=\Delta^{A(\eta)}\left(\gamma_{\eta}, 1\right)$ and the limit variety $T_{\gamma_{\eta}, \Delta_{\eta}} V$ and consider (4).

(4) If there exists $\eta \in M_{1}$, such that $T_{\gamma_{\eta}, \Delta_{\eta}} V$ does not satisfy $\mathrm{PL}_{\text {loc }}(\lambda)$ at any $\lambda \in T_{\gamma_{\eta}, \Delta_{\eta}} V \cap \mathbb{R}^{3}$, then $V$ does not satisfy $\mathrm{PL}_{\text {loc }}(0)$ by Proposition 3.5 . Otherwise consider (5).

(5) If there exist $\eta \in M_{1}$ and $\lambda \in\left(T_{\gamma_{\eta}, \Delta_{\eta}} V\right)_{\text {reg }} \cap \mathbb{R}^{3}$ such that $V$ is not $\left(\gamma_{\eta}, \Delta_{\eta}\right)$ hyperbolic at $\lambda$, then $V$ does not satisfy $\mathrm{PL}_{\text {loc }}(0)$ by Theorem 3.13 . Otherwise consider (6).

(6) If for each $\eta \in M_{1}$, each $\lambda \in\left(T_{\gamma_{\eta}, \Delta_{\eta}} V\right)_{\operatorname{sing}} \cap \mathbb{R}^{3}$ is a simple point of $T_{\gamma_{\eta}, \Delta_{\eta}} V$, then $V$ satisfies $\mathrm{PL}_{\mathrm{loc}}(0)$ by Theorem 5.3. The reason is that by section 5.1 we have

$$
\mathcal{C}_{1}=\left\{\left(\gamma_{\eta}, \Delta_{\eta}\right): \eta \in M_{1}\right\}
$$

so that the present assumption implies $\mathcal{C}_{2}=\emptyset$, hence $\mathcal{C}=\mathcal{C}_{1}$. Consequently, it follows from (1)-(5) that all the conditions in 5.3(c) are fulfilled. Otherwise let

$\mathcal{C}_{1, c}:=\left\{(\gamma, d) \in \mathcal{C}_{1}:\right.$ there exists $\zeta \in\left(T_{\gamma, d} V\right)_{\operatorname{sing}} \cap \mathbb{R}^{3}, \zeta$ is not simple $\}$

and define for $(\gamma, d) \in \mathcal{C}_{1, c}$ and the tangent $\eta$ to $\gamma$ at zero

$$
M_{\gamma, d}:=\left\{\zeta \in\left(T_{\gamma, d} V\right)_{\operatorname{sing}} \cap \mathbb{R}^{3}:\langle\zeta, \eta\rangle=0 \text { and } \zeta \text { is not simple }\right\} .
$$

For $\zeta \in M_{\gamma, d}$ define $\gamma_{\zeta}$ as in section 5.1, let $A(\gamma, \zeta):=T_{\zeta}\left(T_{\gamma, d} V\right) \cap T_{0} V$ and determine $\Delta(\gamma, d, \zeta):=\Delta^{A(\gamma, \zeta)}\left(\gamma_{\zeta}, d\right)$. Then consider $(7)$.

(7) If there are $(\gamma, d) \in \mathcal{C}_{1}$ and $\zeta \in M_{\gamma, d}$ such that $T_{\gamma_{\zeta}, \Delta(\gamma, d, \zeta)} V$ does not satisfy $\mathrm{PL}_{\text {loc }}(\lambda)$ at any $\lambda \in T_{\gamma_{\zeta}, \Delta(\gamma, d, \zeta)} V \cap \mathbb{R}^{3}$, then $V$ does not satisfy $\mathrm{PL}_{\text {loc }}(0)$ by Proposition 3.5.

This procedure may now iterate for a while. However, after finitely many steps, we either find an obstruction against $\mathrm{PL}_{\mathrm{loc}}(0)$ or all the hypotheses of Theorem 5.3(c) are fulfilled and hence $V$ satisfies $\mathrm{PL}_{\text {loc }}(0)$ by Theorem 5.3.

The following lemma shows that under certain hypotheses on the limit varieties, $(\gamma, d)$-hyperbolicity holds automatically.

7.2. Lemma. For $r>0$ let $f: B^{n}(0, r) \rightarrow \mathbb{C}$ be a holomorphic function which is real over real points and vanishes at the origin. For $q \in \mathbb{N}$ let $\gamma(t)=\sum_{j=q}^{\infty} a_{q} t^{j / q}$ be a real simple curve and let $d \in \mathbb{Q}, d \geq 1$ be given. For $\omega_{0}:=\omega_{0}(d)$ defined as in Definition 2.10 consider the expansion of $f(\gamma(t)+z)$ from (2.2) and assume that

$$
\frac{\partial F_{\omega_{0}}}{\partial z_{n}}(1, \xi) \neq 0 \text { for some } \xi \in V\left(F_{\omega_{0}}(1, \cdot)\right) \cap \mathbb{R}^{n} .
$$

Then $V=V(f)$ is $(\gamma, d)$-hyperbolic at $\xi$ with respect to the projection $\pi: \mathbb{C}^{n} \rightarrow \mathbb{C}^{n}$, $\pi\left(z^{\prime}, z_{n}\right):=\left(z^{\prime}, 0\right)$.

Proof. Choose $l \in \mathbb{N}$ such that $d l \in \mathbb{N}$ and such that $q$ divides $l$. Then there is $\varepsilon>0$ such that

$$
h: B^{n}(\xi, \varepsilon) \times B^{1}(0, \varepsilon) \rightarrow \mathbb{C}, h(z, u):=F_{\omega_{0}}(1, z)+\sum_{\omega>\omega_{0}} u^{l\left(\omega-\omega_{0}\right)} F_{\omega}(1, z)
$$


is a holomorphic function. This follows from the convergence properties of the series $\sum_{\omega>\omega_{0}} F_{\omega}$ and the fact that by the choice of $l$ and $\omega_{0}, l\left(\omega-\omega_{0}\right)$ is a natural number for each $\omega$ for which $F_{\omega} \not \equiv 0$. Note that $h$ is real over real points, since $f$ has this property. Obviously, the hypothesis implies

$$
\frac{\partial h}{\partial z_{n}}(0, \xi)=\frac{\partial F_{\omega_{0}}}{\partial z_{n}}(1, \xi) \neq 0 .
$$

Hence the implicit function theorem implies the existence of $\rho>0, \delta>0$, and of a holomorphic function $g: B^{n-1}\left(\xi^{\prime}, \rho\right) \times B^{1}(0, \rho) \rightarrow \mathbb{C}$ such that

$$
\begin{aligned}
V(h) \cap\left(B^{n-1}\left(\xi^{\prime}, \rho\right) \times\right. & \left.B^{1}\left(\xi_{n}, \delta\right) \times B^{1}(0, \rho)\right) \\
& =\left\{\left(z^{\prime}, g\left(z^{\prime}, u\right), u\right):\left(z^{\prime}, u\right) \in B^{n-1}\left(\xi^{\prime}, \rho\right) \times B^{1}(0, \rho)\right\} .
\end{aligned}
$$

Since $h$ is real over real points, we can apply the real implicit function theorem to get that $g$ is real over real points.

To show that $V$ is $(\gamma, d)$-hyperbolic at $\xi$ with respect to $\pi$, let $D:=B^{n-1}(0, \rho) \times$ $B^{1}(0, \delta)$ and fix $\zeta \in V \cap \Gamma\left(\gamma, d, \xi+D, \rho^{l}\right)$ and assume $\pi(\zeta)$ is real. Then there are $0<t<\rho^{l}$ and $w \in D$ such that $\zeta=\gamma(t)+t^{d}(\xi+w)$ and $\pi(w)=\left(w^{\prime}, 0\right) \in \mathbb{R}^{n}$. Moreover, $\zeta \in V$ and the $d$-quasihomogeneity of the functions $F_{\omega}$ imply for $u:=t^{1 / l}$

$$
\begin{aligned}
0=f(\zeta)= & F_{\omega_{0}}\left(t, t^{d}(\xi+w)\right)+\sum_{\omega>\omega_{0}} F_{\omega}\left(t, t^{d}(\xi+w)\right) \\
& =t^{\omega_{0}}\left(F_{\omega_{0}}(1, \xi+w)+\sum_{\omega>\omega_{0}} t^{\omega-\omega_{0}} F_{\omega}(1, \xi+w)\right)=u^{l \omega_{0}} h(\xi+w, u) .
\end{aligned}
$$

Hence it follows from (7.2) that

$$
(\xi+w, u)=\left(\xi^{\prime}+w^{\prime}, g\left(\xi^{\prime}+w^{\prime}, u\right), u\right) .
$$

Since $\xi^{\prime}+w^{\prime}$ and $u$ are real, $g\left(\xi^{\prime}+w^{\prime}, u\right)=\xi_{n}+w_{n}$ is also real. This implies that $\zeta$ is real. Therefore the proof is complete if $\pi$ is noncharacteristic for $T_{\gamma, d} V$ at $\xi$. However, this is the case since $T_{\gamma, d} V=V\left(F_{\omega_{0}}(1, \cdot)\right)$ implies

$$
T_{\xi}\left(T_{\gamma, d} V\right)=\left\{\zeta \in \mathbb{C}^{n}:\left\langle\zeta, \operatorname{grad} F_{\omega_{0}}(1, \xi)\right\rangle=0\right\},
$$

and consequently

$$
\operatorname{ker} \pi \cap T_{\xi}\left(T_{\gamma, d} V\right)=\{0\}
$$

Next we show that under an additional hypothesis, the characterizing conditions in Theorem 5.3 simplify considerably.

7.3. Theorem. Let $V$ be an analytic surface in $\mathbb{C}^{3}$ which contains the origin and which is the zero set of an analytic function with real Taylor coefficients. Assume that $T_{0} V$ and $T_{\gamma, d} V$ have multiplicity 1 for each $(\gamma, d) \in \mathcal{C}(\mathcal{C}$ as defined in section 5.1). Then $V$ satisfies $\mathrm{PL}_{\text {loc }}(0)$ if and only if $T_{0} V$ has $\mathrm{PL}_{\mathrm{loc}}(0)$ and for each $(\gamma, d) \in \mathcal{C}$, the limit variety $T_{\gamma, d} V$ satisfies $\mathrm{PL}_{\text {loc }}(\xi)$ at each real point $\xi$.

Proof. Note first that for $(\gamma, d) \in \mathcal{C}$ we have $d \in \mathbb{Q}$ by section 5.1. Moreover, the hypotheses imply that $F_{\omega_{0}(d)}(1, \cdot)$ is square-free and that this also holds for $F_{\omega_{0}(1)}(1, \cdot)$. Hence it follows from Lemma 7.2 that the hyperbolicity conditions in section 7.1 are all satisfied. Therefore, the theorem follows from section 7.1 (or rather Theorem 5.3). 
Remark. Note that Theorem 7.3 essentially reduces the dimension of the varieties that have to be checked. For $d>1$, this follows from Theorem 2.7(c). Hence we can apply Proposition 3.16 in this case. Since $T_{0} V$ is a homogeneous variety, it follows from Meise, Taylor, and Vogt [26, Theorem 3.13, that $T_{0} V$ satisfies $\mathrm{PL}_{\text {loc }}(0)$ if and only if $V$ satisfies the dimension condition and $\mathrm{PL}_{\text {loc }}(\xi)$ for each $\xi \in T_{0} V \cap S^{2}$. The latter condition can be checked using Braun [5], Corollary 12, or Lemma 6.1 and Proposition 3.16.

The following proposition can be considered as an example which shows that our methods can also be used to prove positive results for certain hypersurfaces in $\mathbb{C}^{n}$ for $n>3$.

7.4. Proposition. Let $f: B^{n}(0, r) \rightarrow \mathbb{C}$ be holomorphic for some $r>0$. Assume that $f$ is real over real points, and that the localization $f_{0}$ of $f$ at zero has no elliptic factors and satisfies $\operatorname{grad} f_{0}(x) \neq 0$ for each $x \in \mathbb{R}^{n} \backslash\{0\}$. Then $V=V(f)$ satisfies $\mathrm{PL}_{\mathrm{loc}}(0)$.

Proof. It is easy to check that the present hypotheses imply the hypotheses of $[8$, Corollary 13. Hence $V$ satisfies $\mathrm{RPL}_{\mathrm{loc}}(0)$, i.e., condition 5.13(a).

Next note that for each $\xi \in T_{0} V \cap \mathbb{R}^{n}=V\left(f_{0}\right) \cap \mathbb{R}^{n}$ with $|\xi|=1$ and $\gamma_{\xi}: t \mapsto t \xi$, $t>0$, the origin belongs to $T_{\gamma_{\xi}, 1} V=T_{0} V-\xi$. Since $\operatorname{grad} f_{0}(\xi) \neq 0$, Lemma 7.2 implies the existence of some zero neighborhood $G_{\xi}$ in $\mathbb{C}^{n}$ and $\rho_{\xi}>0$ such that $V$ is $\left(\gamma_{\xi}, 1\right)$-hyperbolic in $0 \in T_{\gamma_{\xi}, 1} V$. By Lemma 5.7, this implies that $V$ satisfies $\operatorname{PL}\left(V, \Gamma\left(\gamma_{\xi}, 1, G_{\xi}, \rho_{\xi}\right)\right)$. Hence, also condition 5.13(b) is fulfilled. Therefore, $V$ satisfies $\mathrm{PL}_{\text {loc }}(0)$ by Lemma 5.13 .

7.5. Example. Define the polynomials

$$
\begin{aligned}
& P(x, y, z):=y\left(x^{2}-y^{2}\right)+2 x y z^{2}+y z^{4}=P_{0}+P_{1}+P_{2}, \\
& Q(x, y, z):=y\left(x^{2}-y^{2}\right)+x^{2} z^{2} .
\end{aligned}
$$

Then $V(P)$ satisfies $\mathrm{PL}_{\text {loc }}(0)$ while $V(Q)$ fails $\mathrm{PL}_{\text {loc }}(0)$.

To show this we go through the steps in the algorithm of section 7.1, beginning with $V=V(P)$.

(1) By section 2.1 we have

$$
T_{0} V=V\left(P_{0}\right)=\left\{(x, y, z) \in \mathbb{C}^{3}: y\left(x^{2}-y^{2}\right)=0\right\} .
$$

Since $T_{0} V$ is the union of three hyperplanes in $\mathbb{C}^{3}$ which have real generators, $T_{0} V$ satisfies $\mathrm{PL}_{\text {loc }}(0)$. It is easy to check that

$$
\left(T_{0} V\right)_{\text {sing }} \cap S^{2}=\{(0,0,1),(0,0,-1)\} .
$$

(2) Let $\zeta:=(1,0,0)$ and define $\gamma: t \mapsto t \zeta$. For $d=1$ we then have

$$
P(\gamma(t)+(x, y, z))=P_{0}(t+x, y, z)+P_{1}(t+x, y, z)+P_{2}(x, y, z)
$$

and hence, in the notation of Definition 2.10, $\omega_{0}=3$ and

$$
F_{\omega_{0}}(t, x, y, z)=y(t+x)^{2}-y^{3} .
$$

Now let $\pi:(x, y, z) \mapsto(x, 0, z)$ and note that

$$
\frac{\partial F_{\omega_{0}}}{\partial y}(1, x, y, z)=(1+x)^{2}-3 y^{2}
$$


From this it follows easily that $\frac{\partial F_{\omega_{0}}}{\partial y}(1, \eta-\zeta) \neq 0$ each $\eta \in\left(T_{0} V\right)_{\text {reg }} \cap S^{2}$. Hence Lemma 7.2 implies that $V$ is $(\gamma, 1)$-hyperbolic at $\eta-\zeta$ for each $\eta \in\left(T_{0} V\right)_{\text {reg }} \cap \mathbb{R}^{3}$.

(3) We claim that for each $\eta \in\left(T_{0} V\right)_{\operatorname{sing}} \cap S^{2}$ the point $\eta-\zeta$ is a simple point of $T_{\gamma, 1} V$. From this and the construction of the set $\mathcal{C}$ in section 5.1 it follows that $\mathcal{C}=\emptyset$. Hence all the conditions in Theorem 5.3(c) are fulfilled by (1) and $(2)$ and consequently $V(P)$ satisfies $\mathrm{PL}_{\mathrm{loc}}(0)$ by Theorem 5.3.

To prove our claim let $\xi:=(0,1,0)$. Then $\xi \notin T_{0} V$. Since $P(x, y, z)=$ $y\left(x+z^{2}\right)^{2}-y^{3}$ and $\frac{\partial P}{\partial \xi}(x, y, z)=\left(x+z^{2}\right)^{2}-3 y^{2}$, it follows that for projections along $\xi$, the branch locus $B_{\xi}$ is the set

$$
B_{\xi}=\left\{\left(-s^{2}, 0, s\right): s \in \mathbb{C}\right\} .
$$

If the curves $\sigma_{ \pm}$are defined as $\sigma_{ \pm}(t):=(0,0, \pm t)$, then $\Gamma\left(\sigma_{ \pm}, 1, B\left(0, \frac{1}{2}\right), 1\right)$ contains only one branch of $B_{\xi} \cap \mathbb{R}^{3}$, which proves our claim.

To show that $V=V(Q)$ fails $\mathrm{PL}_{\mathrm{loc}}(0)$ we consider the limit varieties $T_{\gamma, d} V$ for the curve $\gamma(t):=(0,0, t)$, which parametrizes a subset of $\left(T_{0} V\right)_{\text {sing }} \cap \mathbb{R}^{3}$. From $[9]$, Proposition 4.3 it follows that the first interesting value for $d$ is $d=2$. Using [9], Corollary 3.17 we get

$$
T_{\gamma, 2} V=\left\{(x, y, z) \in \mathbb{C}^{3}: y^{3}-x^{2}(y+1)=0\right\} .
$$

By Proposition 3.16, the variety

$$
W:=\left\{(x, y) \in \mathbb{C}^{2}: y^{3}-x^{2}(y+1)=0\right\}
$$

does not satisfy $\mathrm{PL}_{\text {loc }}(0)$. Hence $T_{\gamma, 2} V$ does not satisfy $\mathrm{PL}_{\mathrm{loc}}(0)$. By Theorem 3.13, this proves that $V(Q)$ does not satisfy $\mathrm{PL}_{\mathrm{loc}}(0)$.

\subsection{Example. Define}

$$
P(x, y, z):=y\left(x^{2}-y^{2}\right)-y z^{3}+z^{5}=P_{0}+P_{1}+P_{2} .
$$

Then $V=V(P)$ satisfies $\mathrm{PL}_{\mathrm{loc}}(0)$.

To prove this by an application of Theorem 7.3, note first that $P_{0}$ is square-free, that $V\left(P_{0}\right)=T_{0} V$ satisfies $\mathrm{PL}_{\mathrm{loc}}(0)$ and that $\left(T_{0} V\right)_{\operatorname{sing}} \cap S^{2}=\left\{\xi_{+}, \xi_{-}\right\}$, where $\xi_{ \pm}:=(0,0, \pm 1)$. Some computation shows that for $\zeta:=(1,0,0)$ and $\gamma: t \rightarrow t \zeta$, $t>0$, the point $\xi_{-}-\zeta$ is a simple point of $T_{\gamma, 1} V$, while $\xi_{+}-\zeta$ is not simple. By the construction in section 5.1 we therefore have $M_{1}=\left\{\xi_{+}\right\}$. Define $\gamma_{+}: t \mapsto t \xi_{+}$, $t>0$. By Proposition 4.11 the first candidate for $\Delta^{A\left(\xi_{+}\right)}\left(\gamma_{+}, 1\right)$ (in the notation of section $7.1(3))$ is $\Delta=\frac{3}{2}$. A computation shows that this is correct. Hence $\mathcal{C}_{1}=\left\{\left(\gamma_{+}, \frac{3}{2}\right)\right\}$. The procedure described in Definition 2.10 and (2.3) (see [9], Corollary 3.17) gives

$$
T_{\gamma_{+} \frac{3}{2}} V=V\left(F_{\omega_{0}}(1, \cdot)\right), \text { where } F_{\omega_{0}}(1, x, y, z)=Q_{+}(x, y)=y\left(x^{2}-y^{2}-1\right) .
$$

Since $\operatorname{grad} Q_{+}(x, y)=\left(2 x y, x^{2}-3 y^{2}-1\right)$, we get

$$
\left(T_{\gamma_{+} \frac{3}{2}} V\right)_{\operatorname{sing}}=\{( \pm 1,0, z): z \in \mathbb{C}\} .
$$

An inspection of the points $( \pm 1,0,0)$ shows that they are simple points of $T_{\gamma_{+}, \frac{3}{2}} V$. Hence Lemma 4.9 (b) implies that all points of $\left(T_{\gamma_{+}, \frac{3}{2}} V\right)_{\text {sing }}$ are simple. Therefore, the definition of $\mathcal{C}$ in section 5.1 gives $\mathcal{C}=\mathcal{C}_{1}=\left\{\left(\gamma_{+}, \frac{3}{2}\right)\right\}$. Since it is easy to check (using Proposition 3.16) that $T_{\gamma_{+}, \frac{3}{2}} V$ satisfies $\mathrm{PL}_{\text {loc }}(\xi)$ at each $\xi \in T_{\gamma_{+}, \frac{3}{2}} V \cap \mathbb{R}^{3}$, 
we have shown that the hypotheses of Theorem 7.3 are fulfilled. Hence $V$ satisfies $\mathrm{PL}_{\text {loc }}(0)$ by Theorem 7.3 .

\subsection{Example. Define}

$$
P(x, y, z):=y\left(x^{2}-y^{2}\right)\left(x^{2}-4 y^{2}\right)+x\left(x^{2}-3 y^{2}\right) z^{3}+z^{8}=P_{0}+P_{1}+P_{2} .
$$

Then $V:=V(P)$ satisfies $\mathrm{PL}_{\mathrm{loc}}(0)$.

To show this, note first that by section 2.1 we have

$$
T_{0} V=\left\{(x, y, z) \in \mathbb{C}^{3}: P_{0}(x, y, z)=0\right\}
$$

and that

$$
\left(T_{0} V\right)_{\operatorname{sing}} \cap S^{2}=\{(0,0,1),(0,0,-1)\} .
$$

Now let $\eta:=(0,0,1)$ and define $\gamma_{ \pm}: t \mapsto \pm t \eta$. Applying the procedure described in Definition 2.10 or using [9], Lemma 6.1, we get for both curves $\gamma_{+}$and $\gamma_{-}$that $p=3$ and $d_{2}=\frac{3}{2}, d_{3}=\frac{5}{3}$ (see Remark 2.11). Moreover,

$$
\begin{gathered}
F_{\omega_{0}\left(\frac{3}{2}\right), \pm}(1,(x, y, z))=y\left(x^{2}-y^{2}\right)\left(x^{2}-4 y^{2}\right) \pm x\left(x^{2}-3 y^{2}\right) \\
F_{\omega_{0}\left(\frac{5}{3}\right), \pm}(1,(x, y, z))=x\left(x^{2}-3 y^{2}\right)+1 .
\end{gathered}
$$

Since 0 is not a simple point of $T_{\gamma_{ \pm}, 1} V$, we get $M_{1}=\{\eta,-\eta\}$, in the notation of section 5.1. For $A( \pm \eta):=T_{ \pm \eta}\left(T_{0} V\right) \cup T_{0} V$ we get from Lemma 4.11 that $\Delta^{A( \pm \eta)}\left(\gamma_{ \pm}, 1\right) \geq \frac{3}{2}$. A computation shows $\Delta^{A( \pm \eta)}\left(\gamma_{ \pm}, \frac{3}{2}\right)=\frac{3}{2}$ and hence

$$
\mathcal{C}_{1}=\left\{\left(\gamma_{+}, \frac{3}{2}\right),\left(\gamma_{-}, \frac{3}{2}\right)\right\}
$$

Again it turns out that $0 \in T_{\gamma_{ \pm}, \frac{3}{2}} V$ is not simple and using Lemma 4.11 as above we get $\mathcal{C}_{2}=\left\{\left(\gamma_{+}, \frac{5}{3}\right),\left(\gamma_{-}, \frac{5}{3}\right)\right\}$. Since all points in $T_{\gamma_{ \pm}, \frac{5}{3}} V=V\left(F_{\omega_{0}(5 / 3), \pm}(1, \cdot)\right)$ are regular, section 5.1 implies

$$
\mathcal{C}=\left\{\left(\gamma_{+}, \frac{3}{2}\right),\left(\gamma_{-}, \frac{3}{2}\right),\left(\gamma_{+}, \frac{5}{3}\right),\left(\gamma_{-}, \frac{5}{3}\right)\right\}
$$

From (7.3), (7.4), and (7.5) it is obvious that all relevant limit varieties of $V$ have multiplicity 1 so that we can apply Theorem 7.3. By this theorem, $V$ satisfies $\mathrm{PL}_{\text {loc }}(0)$ if $T_{0} V$ satisfies $\mathrm{PL}_{\text {loc }}(0)$ and if $T_{\gamma, d} V$ satisfies $\mathrm{PL}_{\text {loc }}(\xi)$ for each $\xi \in T_{\gamma, d} V \cap$ $\mathbb{R}^{3}$ and each $(\gamma, d) \in \mathcal{C}$.

To check these conditions, note first that $T_{0} V$ is the union of five hyperplanes in $\mathbb{C}^{3}$ which all have real generators. Thus $T_{0} V$ satisfies $\mathrm{PL}_{\text {loc }}(0)$. In the other cases we have $T_{\gamma, d} V=W \times \mathbb{C}$, where $W \subset \mathbb{C}^{2}$ is $V\left(F_{\omega_{0}(d)}(1,(x, y, 0))\right.$. Some computation shows that $W$ is locally hyperbolic at each $\kappa \in W \cap \mathbb{R}^{2}$. This implies that the hypotheses of Theorem 7.3 are fulfilled. Hence $V$ satisfies $\mathrm{PL}_{\mathrm{loc}}(0)$.

Next we consider some examples for the results in Section 6 .

7.8. Example. If $P \in \mathbb{R}[x, y, z, w]$ is defined as

$$
P(x, y, z, w):=x^{4}+y^{4}-y z^{3}+\left(x^{2}-y^{2}\right) y w,
$$

then $P(D): \mathcal{A}\left(\mathbb{R}^{4}\right) \rightarrow \mathcal{A}\left(\mathbb{R}^{4}\right)$ is surjective. In fact, $P(D): \mathcal{D}^{\prime}\left(\mathbb{R}^{4}\right) \rightarrow \mathcal{D}^{\prime}\left(\mathbb{R}^{4}\right)$ admits a continuous linear right inverse. 
To prove this, note first that

$$
\operatorname{grad} P(x, y, z, w)=\left(4 x^{3}+2 x y w, 4 y^{3}-z^{3}-3 y^{2} w, 3 y z^{2},\left(x^{2}-y^{2}\right) y\right)
$$

and hence

$$
V(P)_{\text {sing }}=\{(0,0,0, w): w \in \mathbb{C}\} .
$$

In the notation of section 6.5 this implies by Lemma 6.6 that

$$
V_{3}=V(P) \backslash V(P)_{\text {sing }}, \quad V_{2}=\emptyset, \quad V_{1}=V(P)_{\text {sing }} \backslash\{(0,0,0,0)\} .
$$

To apply Theorem 6.9 note first that $P$ has real coefficients and satisfies grad $P(\xi) \neq$ 0 for each $\xi \in V_{3} \cap \mathbb{R}^{4}$. This implies that $V(P)$ satisfies $P L_{\text {loc }}(\xi)$ for each $\xi \in V_{3} \cap \mathbb{R}^{4}$ (compare Hörmander [17] or the proof of Meise, Taylor, and Vogt [23], Corollary 4.8). Next note that the points $(0,0,0, \pm 1)$ belong to the two different connected components of $V_{1} \cap \mathbb{R}^{4}$. Consider the reductions $Q_{ \pm}$of $P$ in these points, defined by

$$
Q_{ \pm}(x, y, z):=P(x, y, z, \pm 1)=x^{4}+y^{4}-y z^{3} \pm y\left(x^{2}-y^{2}\right) .
$$

A slight variation of Example 7.6 shows that $V\left(Q_{+}\right)$and $V\left(Q_{-}\right)$satisfy $\mathrm{PL}_{\mathrm{loc}}(0)$. Hence Lemma 6.1 implies that $V(P)$ satisfies condition (c) of Theorem 6.9. Consequently, $P(D)$ is surjective on $\mathcal{A}\left(\mathbb{R}^{4}\right)$ by Theorem 6.9 .

Since $P$ is homogeneous, irreducible, and not elliptic, it follows from this and Meise, Taylor, and Vogt [26, Corollary 3.14 that $V(P)$ satisfies the PhragménLindelöf condition $\operatorname{PL}\left(\mathbb{R}^{4}\right)$, which is equivalent to the fact that $P(D): \mathcal{D}^{\prime}\left(\mathbb{R}^{4}\right) \rightarrow$ $\mathcal{D}^{\prime}\left(\mathbb{R}^{4}\right)$ admits a continuous linear right inverse.

7.9. Example. If $P \in \mathbb{R}[x, y, z, w]$ is defined by

$$
P(x, y, z, w):=y\left(x^{2}-y^{2}\right) w^{2}-y z^{3} w+z^{5},
$$

then $P(D): \mathcal{A}\left(\mathbb{R}^{4}\right) \rightarrow \mathcal{A}\left(\mathbb{R}^{4}\right)$ is not surjective.

To prove this, note that some computation shows that

$$
V(P)_{\text {sing }}=(\{(0,0,0)\} \times \mathbb{C}) \cup\left(\mathbb{C}^{2} \times\{(0,0)\}\right) .
$$

Hence the origin is the only singular point of the variety $V(P)_{\text {sing. In order to }}$ decompose $V(P)$ according to section 6.5 as $\bigcup_{j=0}^{3} V_{j}$, we have to determine the set

$$
E:=\left\{z \in V(P)_{\text {sing }}: \operatorname{dim} C_{4}(V(P), z)>3\right\} .
$$

As it is explained in [10, 22, this can be done in an algorithmic way and gives

$$
E=\mathbb{C} \cdot(1,0,0,0) \cup \mathbb{C} \cdot(1,1,0,0) \cup \mathbb{C} \cdot(1,-1,0,0) \cup \mathbb{C} \cdot(0,0,0,1) .
$$

By section 6.5, this implies

$$
V_{3}=V(P) \backslash V(P)_{\text {sing }}, V_{2}=\mathbb{C}^{2} \times\{(0,0)\} \backslash E, V_{1}=E \backslash\{0\}, V_{0}=\{(0,0,0,0)\} .
$$

By Theorem $6.9(\mathrm{~d}), P(D)$ is not surjective on $\mathcal{A}\left(\mathbb{R}^{4}\right)$ if we show that for some reduction $Q$ of $P$ at some point $\xi \in V(P) \cap S^{3}$ the variety $V(Q)$ does not satisfy $\mathrm{PL}_{\mathrm{loc}}(\xi)$. There are several choices for $\xi$ and various ways to argue (compare [10], $23)$. To avoid coordinate changes, we consider the reduction $Q$ of $P$ at $(1,0,0,0)$ defined as

$$
Q(y, z, w):=P(1, y, z, w)=y w^{2}-y^{2} w^{2}-y z^{3} w+z^{5} .
$$


To show that $V(Q)$ does not satisfy $\mathrm{PL}_{\text {loc }}(0)$, define $\gamma: t \mapsto(0,0, t)$. Then it follows from [9], Corollary 3.17 that $T_{\gamma, \frac{5}{3}} V(Q)=W \times \mathbb{C}$, where $W=V\left(Q_{1}\right)$ and

$$
Q_{1}(y, w)=y w^{2}+1 \text {. }
$$

Some computation shows that $V(Q)$ is not $\left(\gamma, \frac{5}{3}\right)$-hyperbolic at $(0,0,0) \in T_{\gamma, \frac{5}{3}} V(Q)$. Hence $V(Q)$ does not satisfy $\mathrm{PL}_{\text {loc }}(0)$ by Theorem 3.13 .

7.10. Example. If $P \in \mathbb{R}\left[x_{1}, \ldots, x_{4}\right]$ is defined as in Langenbruch [20], p. 256, by

$$
P\left(x_{1}, \ldots, x_{4}\right):=x_{1}^{2}\left(x_{2}^{2}\left(x_{3}^{2}+x_{4}^{2}\right)+x_{3}^{4}\right)+x_{2}^{6}+x_{3}^{6}+x_{4}^{6},
$$

then $P(D): \mathcal{A}\left(\mathbb{R}^{4}\right) \rightarrow \mathcal{A}\left(\mathbb{R}^{4}\right)$ is not surjective. This follows from Theorem 6.9 if we show that $V(P):=\left\{z \in \mathbb{C}^{4}: P(z)=0\right\}$ does not satisfy $\operatorname{PL}_{\text {loc }}\left(e_{1}\right)$, for $e_{1}:=(1,0,0,0)$. To prove this, we argue by contradiction and assume that $V(P)$ satisfies $\mathrm{PL}_{\mathrm{loc}}\left(e_{1}\right)$. Then let $e_{1}, \ldots, e_{4}$ denote the canonical basis vectors of $\mathbb{C}^{4}$ and note that by Lemma 6.1 for the reduction $Q$ of $P$ at $e_{1}$, defined by

$$
Q(x, y, z):=P\left(e_{1}+x e_{2}+y e_{3}+z e_{4}\right)=x^{2}\left(y^{2}+z^{2}\right)+z^{4}+x^{6}+y^{6}+z^{6},
$$

the variety $V(Q)$ satisfies $\mathrm{PL}_{\text {loc }}(0)$. By Theorem 5.3(c), this implies that $T_{0} V(Q)=$ $V\left(Q_{0}\right)$ satisfies $\mathrm{PL}_{\mathrm{loc}}(0)$, where $Q_{0}(x, y, z)=x^{2}\left(y^{2}+z^{2}\right)+z^{4}$. Now let $\xi:=$ $(1,0,0) \in V\left(Q_{0}\right)$ and note that by Proposition 3.5 and Theorem 2.7(b) the variety $V\left(Q_{0}\right)$ satisfies $\mathrm{PL}_{\mathrm{loc}}(\xi)$. Applying Lemma 6.1 again, we get that the reduction $R$ of $Q_{0}$ at $(1,0,0)$, defined by

$$
R(y, z)=Q_{0}(1, y, z)=y^{2}+z^{2}+z^{4},
$$

satisfies $\mathrm{PL}_{\mathrm{loc}}(0)$. This, however, is not the case since the localization $R_{0}$ of $R$ at the origin is elliptic. From this contradiction it follows that our assumption was false, hence $V(P)$ does not satisfy $\mathrm{PL}_{\mathrm{loc}}\left(e_{1}\right)$.

In conclusion we remark that we do not know of any examples of varieties of dimension three or greater that are hyperbolic in conoids but fail to satisfy $\mathrm{PL}_{\text {loc }}(0)$. Therefore it might be reasonable to conjecture that hyperbolicity in conoids gives the characterization for $\mathrm{PL}_{\mathrm{loc}}(0)$ for varieties in all dimensions.

\section{REFERENCES}

[1] Andersson K.G.: Propagation of analyticity of solutions of partial differential equations with constant coefficients, Ark. Mat. 8 (1971), 277-302. MR 45:8986

[2] Andreotti, A., Nacinovich, M.: Analytic convexity and the principle of Phragmén-Lindelöf, Scuola Norm. Sup. Pisa, (1980). MR 83h:32018

[3] Bochnak, J., Coste, M., and Roy, M.-F.: Géometrie algébrique réelle, Ergebnisse Math. Grenzgebiete, 3. Folge, Band 12, Springer, Berlin 1987.

[4] Boiti, C., Nacinovich, M.: The overdetermined Cauchy problem, Ann. Inst. Fourier (Grenoble) 47 (1997), 155-199. MR 98a:35095

[5] Braun, R. W.: Hörmander's Phragmén-Lindelöf principle and irreducible singularities of codimension 1, Boll. Un. Mat. Ital. (7) 6-A (1992), 339-348. MR 94b:35012

[6] Braun, R. W.: The surjectivity of a constant coefficient homogeneous differential operator on the real analytic functions and the geometry of its symbol, Ann. Inst. Fourier (Grenoble), 45 (1995), 223-249. MR 96e:35025

[7] Braun, R.W., Meise, R., Taylor, B.A.: Algebraic varieties on which the classical PhragménLindelöf estimates hold for plurisubharmonic function, Math. Z. 232 (1999), 103-135. MR 2001d:32048

[8] Braun, R.W., Meise, R., Taylor, B.A.: Local radial Phragmén-Lindelöf estimates for plurisubharmonic functions on analytic varieties, Proc. Amer. Math. Soc. 131 (2003), 2423-2433. 
[9] Braun, R.W., Meise, R., Taylor, B.A.: Higher order tangents to analytic varieties along curves, Canad. J. Math. 55 (2003), 64-90.

[10] Braun, R.W., Meise, R., Taylor, B.A.: Surjectivity of constant coefficient partial differential operators on $\mathcal{A}\left(\mathbb{R}^{4}\right)$ and Whitney's $C_{4}$-cone, Bull. Soc. R. Sci. Liège 70 (2001), 195-206. MR 2003f: 35039

[11] Braun, R.W., Meise, R., Taylor, B.A.: The algebraic surfaces on which the classical Phragmén-Lindelöf theorem holds, manuscript.

[12] Braun, R.W., Meise, R., Vogt, D.: Characterization of the linear partial differential operators with constant coefficients which are surjective on non-quasianalytic classes of Roumieu type on $\mathbb{R}^{N}$, Math. Nachrichten 168 (1994), 19-54. MR 95g:35004

[13] Cartan, H.: Variétés analytiques réelles et variétés analytiques complexes. Bull. Soc. Math. France 85 (1957), 77-99. MR 20:1339

[14] Chirka, E.M.: Complex Analytic sets, Kluwer, Dordrecht, 1989. MR 92b:32016

[15] Franken, U., Meise, R.: Extension and lacunas of solutions of linear partial differential equations, Ann. Inst. Fourier (Grenoble) 46 (1996), 429-464. MR 97h:35005

[16] Franken, U., Meise, R.: Extension and lacunas of solutions of linear partial differential equations on ultradifferentiable functions of Beurling type, in 'Structure of Solutions of Differential Equations', M. Morimoto, T. Kawai (Eds.), World Scientific (1996), 103-127. MR 98g:35035

[17] Hörmander, L.: On the existence of real analytic solutions of partial differential equations with constant coefficients, Invent. Math. 21 (1973), 151-183. MR 49:817

[18] Kaneko, A.: Hartogs type extension theorem of real analytic solutions of linear partial differential equations with constant coefficients, in Advances in the Theory of Fréchet Spaces, T. Terzioğlu (Ed.) NATO ASI Series C, Vol. 289 (Kluwer), 1989, 63-72.

[19] Klimek, M.: Pluripotential Theory, Oxford Science Publications, 1991. MR 93h:32021

[20] Langenbruch, M.: Surjective partial differential operators on real analytic functions defined on open convex sets, Manuscr. Math. 103 (2000), 241-263. MR 2001k:35050

[21] Langenbruch, M.: Characterization of surjective partial differential operators on spaces of real analytic functions, preprint.

[22] Meise, R., and Taylor, B.A.: Phragmén-Lindelöf conditions for graph varieties, Result. Math. 36 (1999), 121-148. MR 2000j:32057

[23] Meise, R., Taylor, B.A., Vogt, D.: Characterization of the linear partial differential operators with constant coefficients that admit a continuous linear right inverse, Ann. Inst. Fourier (Grenoble) 40 (1990), 619-655. MR 92e:46083

[24] Meise, R., Taylor, B.A., Vogt, D.: Extremal plurisubharmonic functions of linear growth on algebraic varieties, Math. Z. 219 (1995), 515-537. MR 96j:32019

[25] Meise, R., Taylor, B.A., Vogt, D.: Continuous linear right inverses for partial differential operators on non-quasianalytic classes and on ultradistributions, Math. Nachr. 180 (1996), 213-242. MR 97j:46036

[26] Meise, R., Taylor, B.A., Vogt, D.: Phragmén-Lindelöf principles on algebraic varieties, J. Amer. Math. Soc. 11 (1998), 1-39. MR 98j:32007

[27] Momm, S.: On the dependence of analytic solutions of partial differential equations on the right-hand side, Trans. Amer. Math. Soc. 345 (1994), 729-752. MR 95a:46036

[28] Nevanlinna, R.: Eindeutige analytische Funktionen, Springer, Berlin, Heidelberg, New York 1974. MR 49:9165

[29] Palamodov, V.P.: A criterion for splitness of differential complexes with constant coefficients, in 'Geometrical and Algebraical Aspects in Several Complex Variables', C.A. Berenstein and D.C. Struppa (Eds.), EditEl (1991), pp. 265-290. MR 94d:58137

[30] Siciak, J.: Extremal plurisubharmonic functions in $\mathbb{C}^{n}$, Ann. Polon. Math. 39 (1981), 177211. MR 83e:32018

[31] Stutz, J.: Analytic sets as branched coverings, Trans. Amer. Math. Soc. 166 (1972), 241-259. MR 48:2420

[32] Whitney, H.: Complex analytic varieties, Addison-Wesley Pub. Co., 1972. MR 52:8473

[33] Zampieri, G.: Propagation of singularity and existence of real analytic solutions of locally hyperbolic equations, J. Fac. Sci. Univ. Tokyo, Sect. I A, Math. 31 (1984), 373-390. MR 86c:58142

[34] Zampieri, G.: An application of the fundamental principle of Ehrenpreis to the existence of global solutions of linear partial differential equations, Boll. U. M. I. 6 (1986), 361-392. MR 88a:35044 
[35] Zariski, O.: Studies in equisingularity I, equivalent singularities of plane algebroid curves, Amer. J. Math. 97 (1965), 507-536. MR 31:2243

[36] Zeriahi, A.: Fonction de Green pluricomplexe à pole à l'infini sur un espace de Stein parabolique et applications, Math. Scand. 69 (1991), 89-126. MR 93a:32021

Mathematisches Institut, Heinrich-Heine-Universität, Universitätsstrasse 1, 40225 DÜsSELdorf, Germany

E-mail address: Ruediger.Braun@uni-duesseldorf.de

Mathematisches Institut, Heinrich-Heine-Universität, Universitätsstrasse 1, 40225 Düsseldorf, Germany

E-mail address: meise@cs.uni-duesseldorf.de

Department of Mathematics, University of Michigan, Ann Arbor, Michigan 48109

E-mail address: taylor@umich.edu 